\title{
Self-Healing Capability of Engineered Cementitious Composites Incorporating Different Types of Pozzolanic Materials
}

By

\author{
Ahmed Alyousif \\ B.Sc., Mosul University, Iraq 2007 \\ A Dissertation \\ Presented to \\ Ryerson University \\ In partial fulfillment for the degree of \\ Doctor of Philosophy \\ Civil Engineering
}

M.Sc., Eastern Mediterranean University, Northern Cyprus, Turkey 2010

Toronto, Ontario, Canada, 2016

(C) Ahmed Alyousif, 2016 


\section{Author's Declaration}

I hereby declare that I am the sole author of this dissertation. This is a true copy of the dissertation, including any required final revisions, as accepted by my examiners.

I authorize Ryerson University to lend this dissertation to other institutions or individuals for the purpose of scholarly research

I further authorize Ryerson University to reproduce this dissertation by photocopying or by other means, in total or in part, at the request of other institutions or individuals for the purpose of scholarly research.

I understand that my dissertation may be made electronically available to the public. 


\title{
Self-Healing Capability of Engineered Cementitious Composites Incorporating Different Types of Pozzolanic Materials
}

\author{
Ahmed Alyousif \\ Doctor of Philosophy, Civil Engineering \\ Ryerson University, Toronto, Canada, 2016
}

\begin{abstract}
Engineered cementitious composites (ECCs) are relatively new construction materials characterized by strain-hardening behavior under excessive tensile loading. The intrinsic selfhealing ability of cracks in ECC is an attractive methodology for durability and in some cases for regaining of mechanical properties. In order to achieve the robust self-healing criteria, this dissertation comprises of four main parts. The first part explores the effectiveness of frost action on water movement into micro-cracked ECC. Experimental findings show that until the end of 150 freezing and thawing $(\mathrm{F} / \mathrm{T})$ cycles, sorptivity measurements of ECC went down due to self-healing in micro-cracks. However, after $150 \mathrm{~F} / \mathrm{T}$ cycles, deterioration surpasses the self-healing effect and increased sorptivity. The second part of the research focuses on the effect of self-healing capability on the different transport properties of micro-cracked ECC with several maturity levels and incorporating different mineral admixtures. Experimental results reveal that with the selection of proper mineral admixture, 92\% recovery in water sorptivity results is attainable mostly in the first seven days of curing. The third part investigates the effects of progressively increasing sustained loading on self-healing behavior of 180-day-old micro-cracked ECC incorporating different mineral admixtures. Experimental results show that even under progressively increasing sustained mechanical loading, modulus of rupture results greater than the original values could be obtained, depending on the mineral admixture selection. The last part of the dissertation investigates the selfhealing behavior of large scale ECC reinforced beams under load influence. The results show that ECC beams with no regards to their size exhibit strength recovery after 90 days of extended moist curing. As for ductility, ECC beams with lower shear span to depth ratio have more pronounced recovery than the ones with higher ratios.
\end{abstract}




\section{Acknowledgments}

I owe my deepest gratitude to my supervisors, Dr. Mohamed Lachemi and Dr. Khandaker M. Anwar Hossain whose inspiration, motivation, guidance and support from the beginning to the end of this study enabled me to develop an understanding of the subject.

I would like to thank Dr. Mustafa Sahmaran from the Civil Engineering Department of Gazi University, Turkey, for his help and guidance during my study.

I am indebted to the whole department of Civil Engineering at Ryerson University, especially to Nidal Jaalouk, Min Yao and Noura Sinno for their time and support during the experimental part of the thesis.

Also, many thanks and appreciation to Firas Almasri, Tony Cicchetti, Christopher Mercieca and Muhammed Anwar (from University of Toronto), for their outstanding help during the experimental part of the thesis.

Finally, I thank with love to my family, my wife Haefa and my daughters Iman and Aya for their everlasting love, support, patience and encouragement throughout my life; this dissertation would have been simply impossible without them. 
To the soul of my beloved father 


\section{Contents}

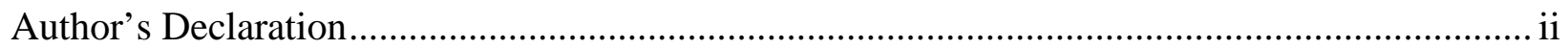

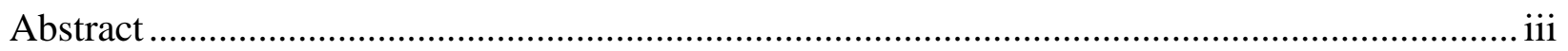

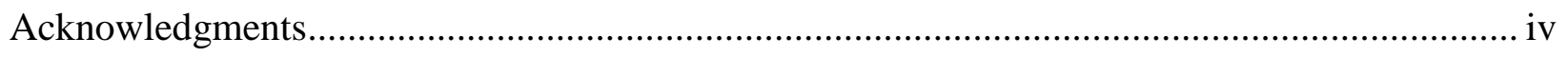

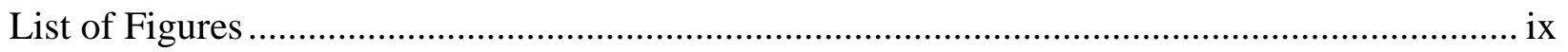

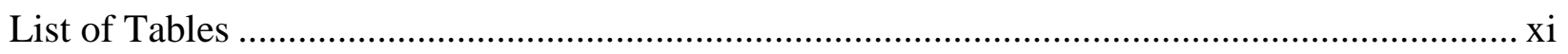

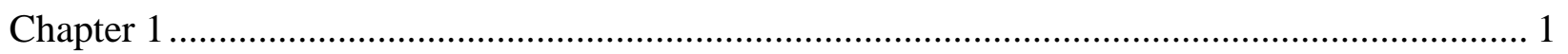

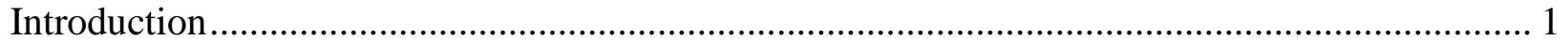

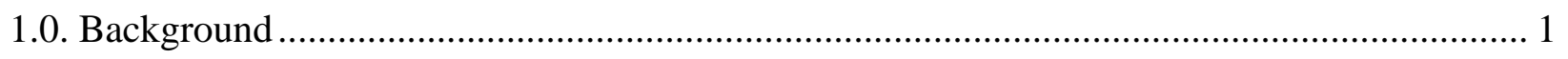

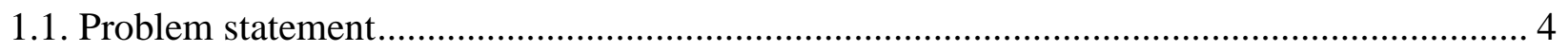

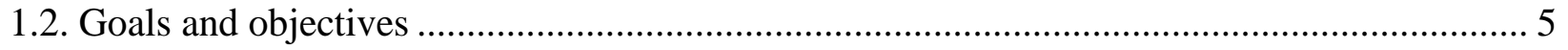

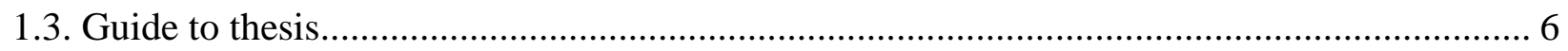

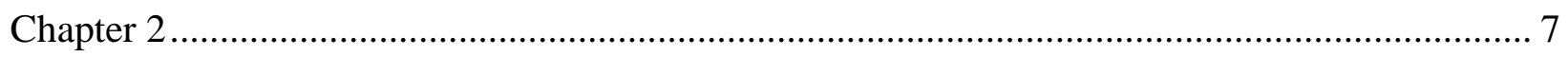

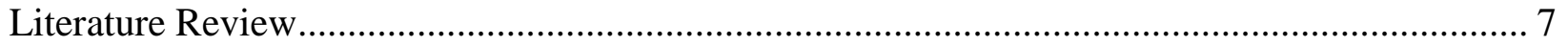

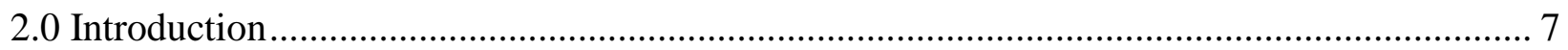

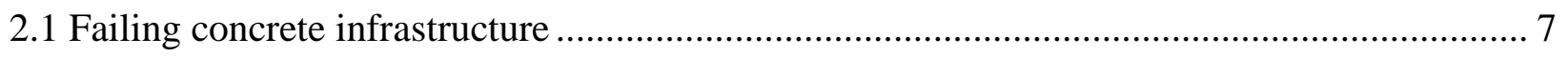

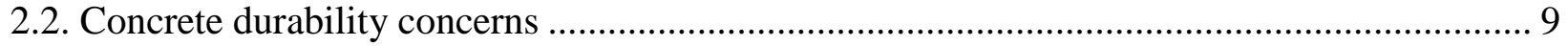

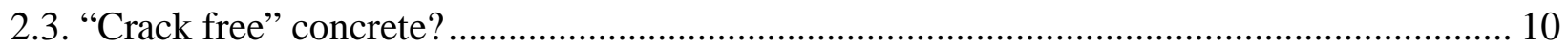

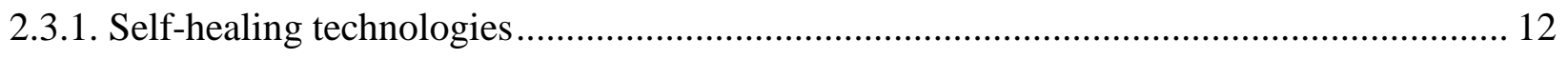

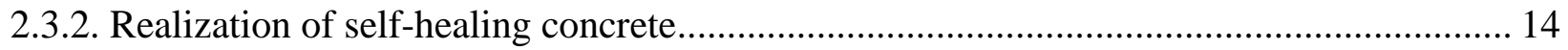

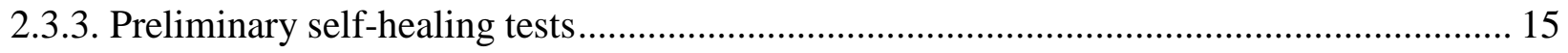

2.4. Design and use of HPFRCC materials for self-healing ................................................ 17

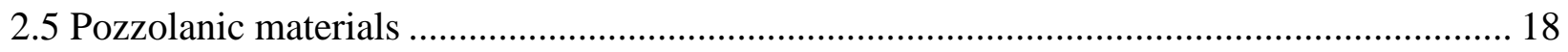

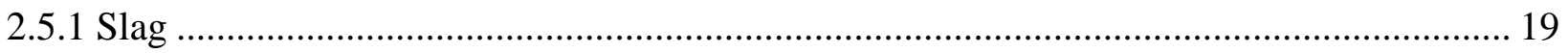

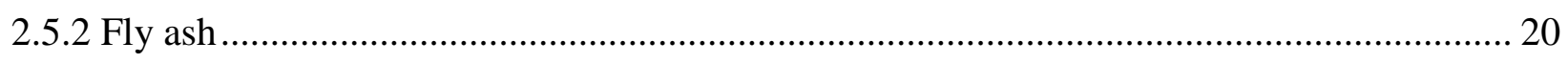

2.6. Intrinsic self-healing capability of ECCs with different types of SCM............................ 21

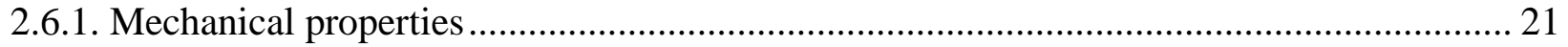

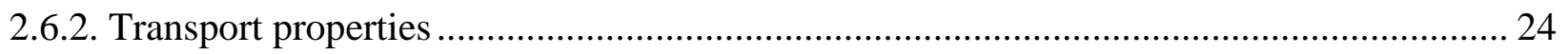

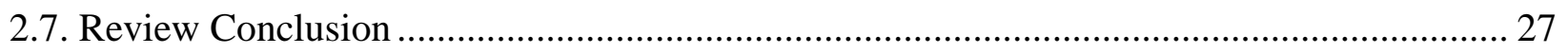

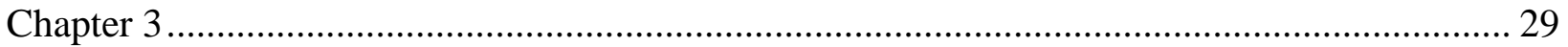


Effects of Microcracking and Type of Cementitious Materials on the Mechanical and Durability

Performance of Cementitious Composites.................................................................. 29

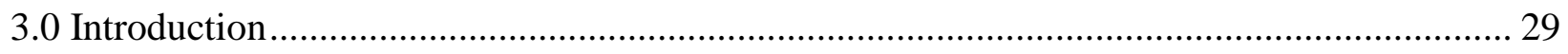

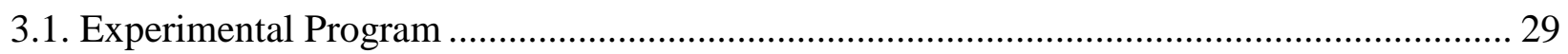

3.1.1. Materials and Mixture Proportions …....................................................................... 29

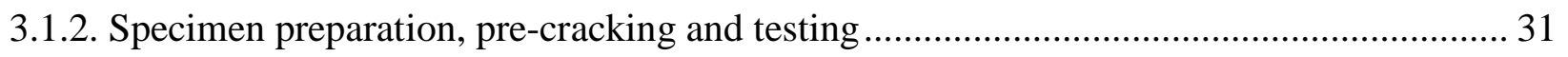

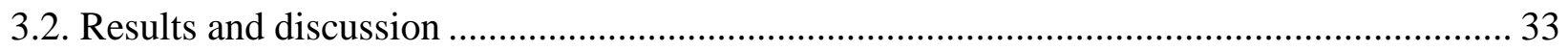

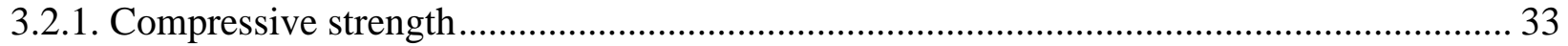

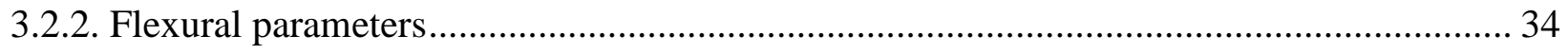

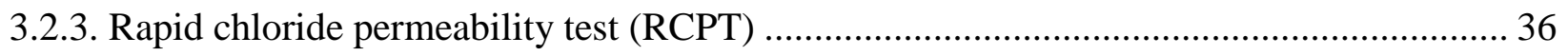

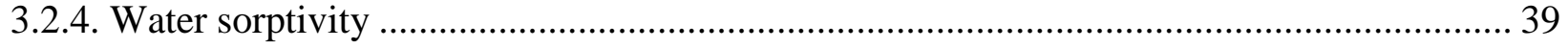

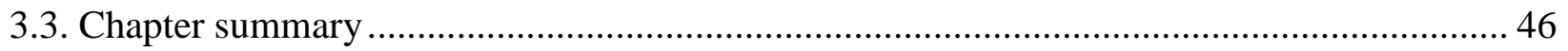

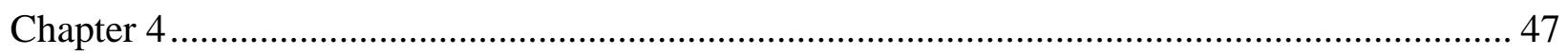

Effect of Self-Healing on the Different Transport Properties of Cementitious Composites incorporating different mineral admixtures .............................................................. 47

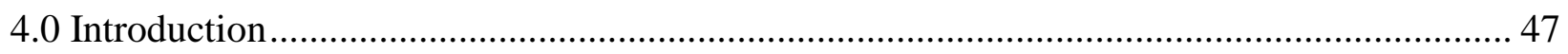

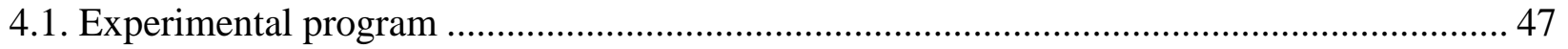

4.1.1. Materials, mixture proportions and basic mechanical properties ................................ 47

4.1.2. Sample preparation, pre-cracking and methods for self-healing evaluation..................... 48

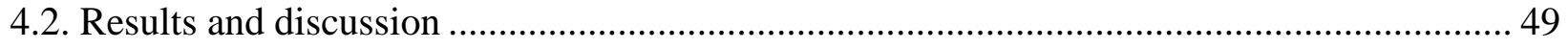

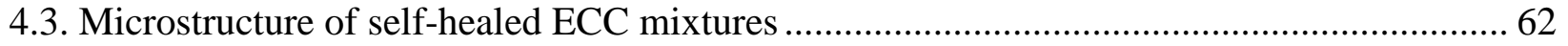

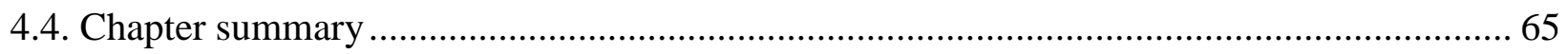

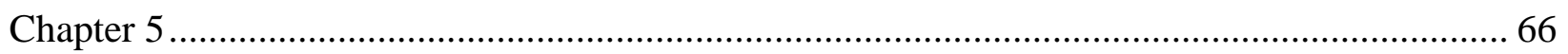

Assessing the Self-Healing Capability of Cementitious Composites under Progressively

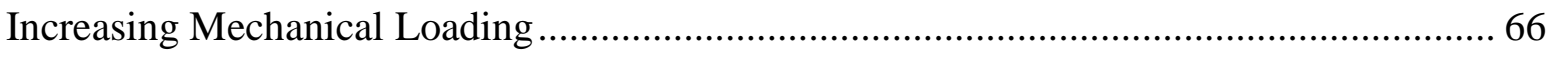

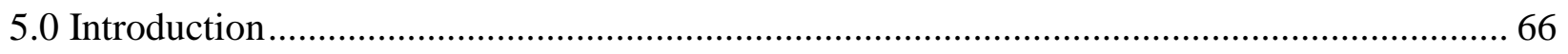

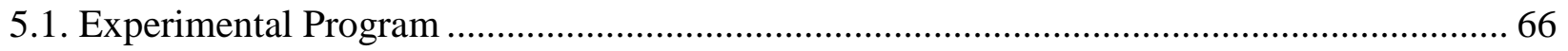

5.1.1. Materials, mixture proportions and basic mechanical properties .................................. 66

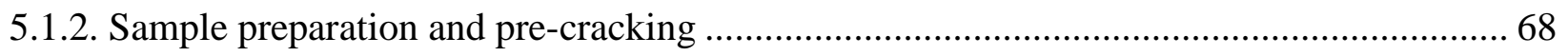

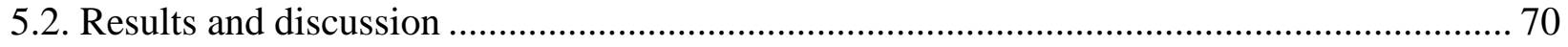

5.2.1. Recovery of mechanical properties........................................................................ 70 
5.2.2. Recovery of ultrasonic pulse velocity (UPV) measurements ...................................... 76

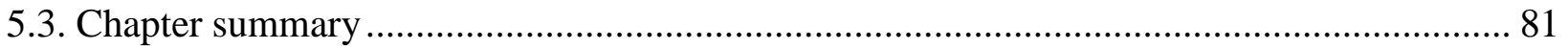

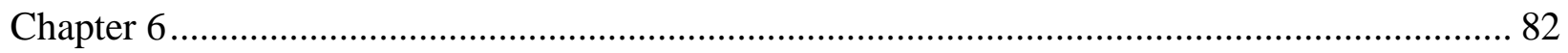

Self-healing behavior of full-scale Cementitious Composites with different shear span to depth

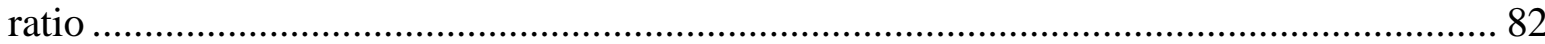

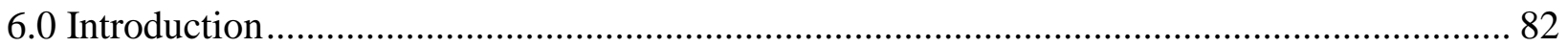

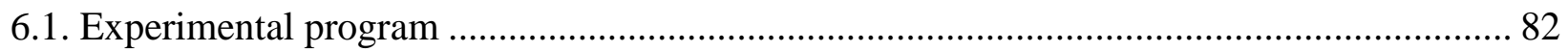

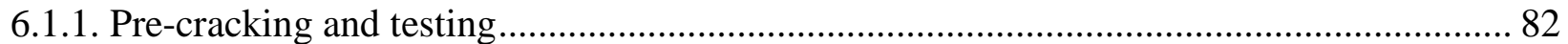

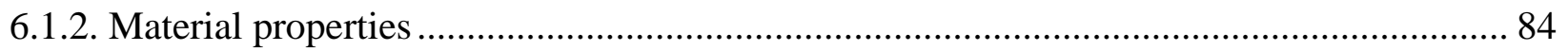

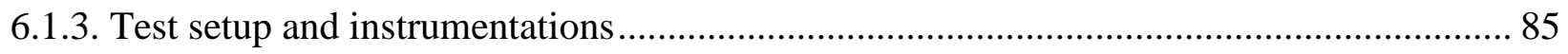

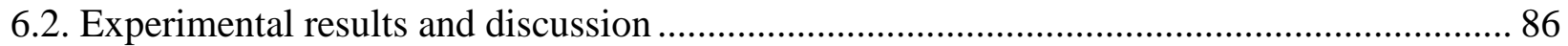

6.2.1. General behavior and failure modes of the virgin specimens at the age of 28 days.......... 86

6.2.2. Strength and stiffness of beams and influence of self-healing ..................................... 88

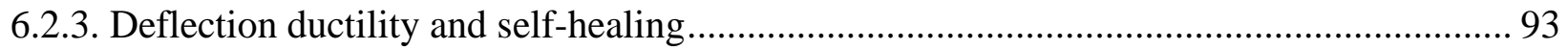

6.2.4 Energy absorption capacity and recovery due to self-healing ........................................ 95

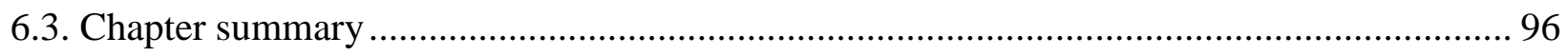

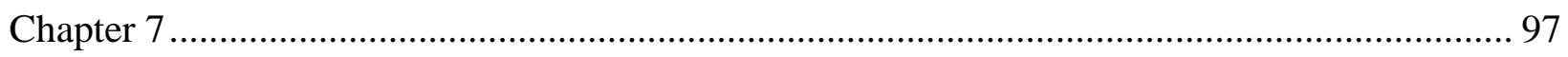

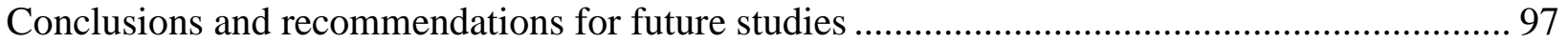

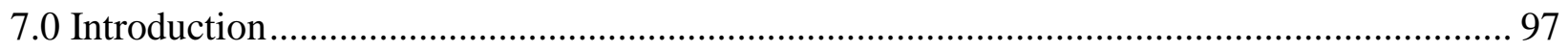

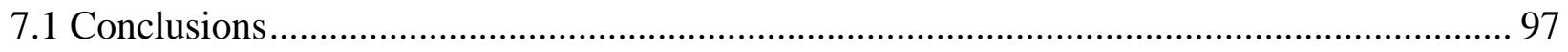

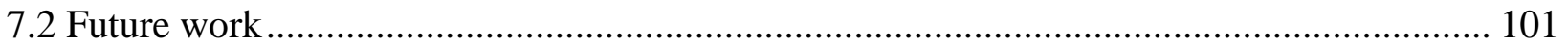

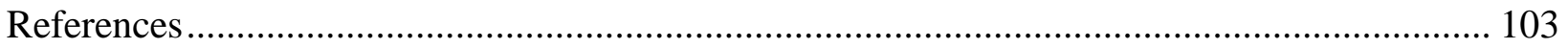




\section{List of Figures}

Figure 2.1: Uniaxial tensile response and crack width development of HPFRCC material......... 15

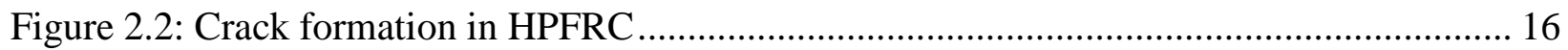

Figure 2.3: Cracks through virgin ECC material adjacent to a self-healed crack held tight by self-

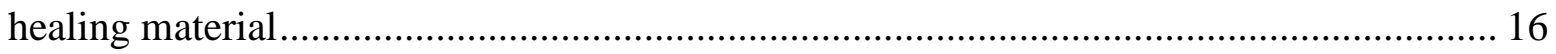

Figure 3.1: Particle size distributions of solid ingredients.................................................... 30

Figure 3.2: Schematic representation of water sorptivity test setup and cut-off prism specimen 33

Figure 3.3: Compressive strength results with respect to different curing ages ........................ 33

Figure 3.4: Formation of multiple microcracks over an ECC cylinder specimen upon exposure to

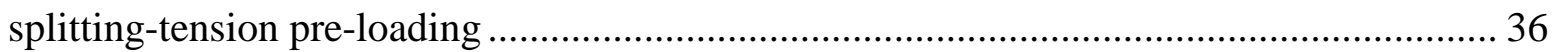

Figure 3.5: Changes in sorptivity results with the number of cracks....................................... 40

Figure 3.6: Percent changes in water sorptivity results of ECCs with different crack numbers

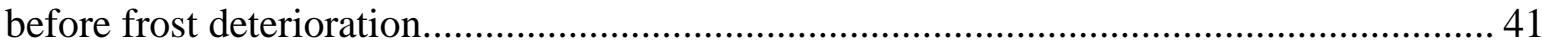

Figure 3.7: ECC_L and ECC_S prisms $(75 \times 75 \times 50 \mathrm{~mm})$ after 6 hours of water exposure .......... 42

Figure 3.8: Water sorptivity measurements after the application of F/T cycles ........................ 43

Figure 4.1: Typical example showing the changes in sorptivity indexes of ECC mixtures with the

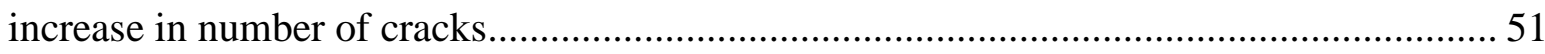

Figure 4.2: Effect of self-healing on sorptivity results of ECCs with: a) no cracks b) min. \# of

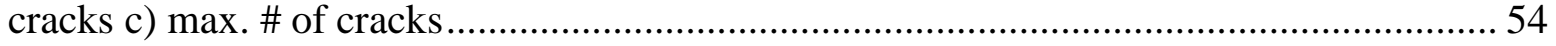

Figure 4.3: Typical self-healing of microcracks after exposure to CW curing .......................... 56

Figure 4.4: Percentage changes in chloride ion penetrability results of pre-loaded ECCs with further CW curing. 61

Figure 4.5: SEM micrograph with EDS pattern of products in self-healed cracks of the surface

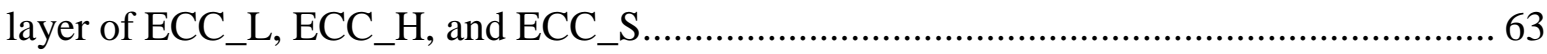

Figure 4.6: XRD paterns of self-healing products in ECC mixtures ....................................... 64

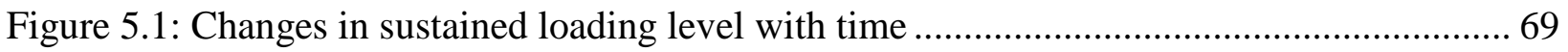

Figure 5.2: Torque wrench, application of sustained loading and curing of specimens.............. 70

Figure 5.3: Calibration of torque wrench against the load cell two weeks before the start of actual tests 70

Figure 5.4: View of microcracked surfaces after exposure to continuous moist curing.............. 73 
Figure 5.5: Percentage variations in UPV results of ECCs with the applied initial pre-loading and further curing a) without b) with progressively increasing sustained loading.................... 79

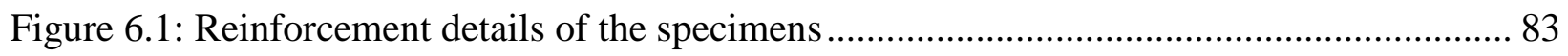

Figure 6.2: Typical flexural stress-deflection graphs of ECC after 28 days.............................. 85

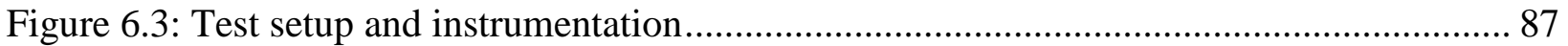

Figure 6.4: Strain measurements of longitudinal tensile reinforcements ................................ 88

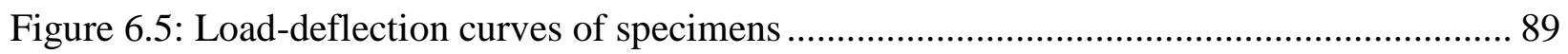

Figure 6.6: Crack distributions and failure modes of ECC specimens .................................... 90

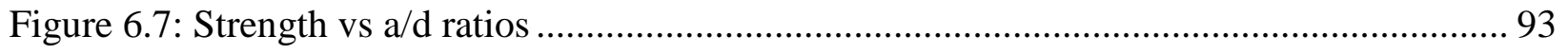

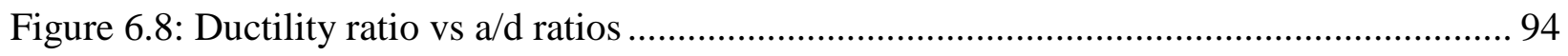

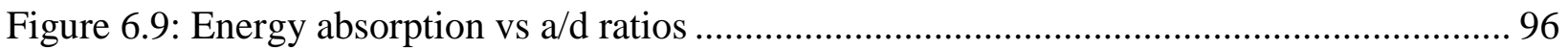




\section{List of Tables}

Table 2.1: Number of cracks and maximum width in HPFRC specimen................................... 16

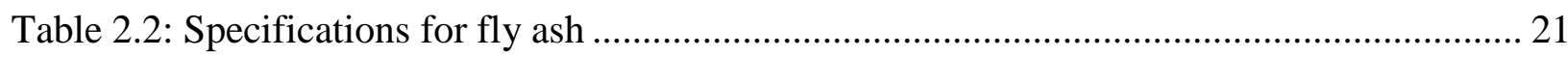

Table 3.1: Chemical and physical properties of PC and different SCM types .......................... 30

Table 3.2: Mixture proportions and compressive strength results of ECC mixtures................... 30

Table 3.3: Flexural parameters of ECC specimens................................................................ 35

Table 3.4: Rapid chloride permeability test results of ECC specimens................................... 36

Table 3.5: Air-void characteristics of different ECCs .............................................................. 38

Table 3.6: Crack characteristics and sorptivity indexes of ECC specimens ............................. 40

Table 4.1: Sorptivity indexes and crack characteristics and of ECC specimens ....................... 50

Table 4.2: Rapid chloride permeability test results of ECC specimens.................................... 58

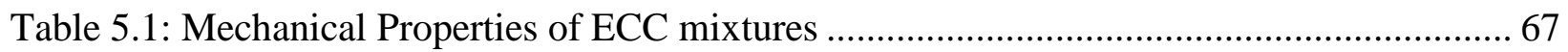

Table 5.2: Flexural properties of ECC mixtures before and after continuous water exposure..... 71

Table 5.3: Ultrasonic pulse velocity (UPV) test results of ECC specimens .............................. 77

Table 6.1: Mixture proportions and basic mechanical properties............................................ 84

Table 6.1: Overall results of the beam specimens after four-point bending tests...................... 91 


\section{Chapter 1}

\section{Introduction}

\subsection{Background}

It is a well-established fact that the performance of concrete mixtures is not solely limited to mechanical property characterization but is also significantly affected by the parameters affecting durability. Durability has tremendous effects on the economy and serviceability of concrete, reinforced concrete and pre-stressed concrete structures, making it a key issue. However, civil infrastructures are often under the influence of environmental impacts and/or mechanical loads that result in drastic changes in durability through cracking. Once crack occurrence takes place, restoring the sound properties of concrete is not an easy task since cracks introduce additional pathways that accelerate the transportation of various media (e.g. liquids, aggressive ions, gaseous substances) into the material. As the transport of those various agents continues, contingency for disruptive mechanisms such as corrosion, sulfate/acid attack, and freeze/thaw susceptibility significantly increases, making the concrete highly vulnerable to fast and serious deterioration. In many instances, crack-originated and further exacerbated deterioration necessitates urgent renovation of the damaged areas. However, repair and/or maintenance applications can be troublesome and the costs involved in renewing deteriorated concrete structures can be high; in extreme cases, even higher than the initial construction cost. In addition to the financial issues, the environmental impacts of the restoration of damaged infrastructures can prevent them from occurring (Witmann, 1998). In this regard, the intrinsic self-healing ability of cracks in concrete material - further favored by the formation of cracks with smaller widths - is an attractive methodology for durability and in some cases for mechanical property regain (Sahmaran et al., 2007; Sahmaran et al., 2013; Sahmaran and Li, 2009).

Concrete is brittle and the brittleness of the material becomes more pronounced in higher-strength grades. This phenomenon has been of interest to many researchers; studies using fibers to address the high brittleness of concrete started in the past decades (Romualdi and Mandel, 1964). Invaluable efforts to modify the brittleness of conventional concrete materials made way for the emergence of new class of materials known as High Performance Fiber Reinforced Cementitious 
Composites (HPFRCC) (Naaman and Reinhardt, 2003). Engineered Cementitious Composites (ECCs), a special kind of HPFRCC, were first introduced about 20 years ago (Li, 1998). The main characteristic of ECCs, differentiating it from conventional fiber reinforced concretes (FRCs), is their high tensile strain capacity and strain-hardening behavior under uniaxial tensile loading, which is supplied through a delicate balance among the individual components of the cementitious system (i.e. fibers, matrix and fiber-to-matrix interface) resulting from the micromechanics-based material design ( $\mathrm{Li}$ et al., 2001). Superior ductility, which is a direct result of high tensile strain capacity and strain-hardening behavior, is realized by the formation of many closely-spaced multiple micro-cracks (widths of less than $100 \mu \mathrm{m}$ ), making ECCs at least 100 times higher in ductility than conventional concrete used for common construction practices (Li, 2003). Taking into account the formation of well-controlled micron-sized cracks in ECCs, they can be regarded as promising materials for enhanced self-healing capability, since tolerable crack widths are reported to be one of the decisive parameters influencing the mechanism (Edvardsen, 1999).

Over the last decade, self-healing phenomena have gained increasing popularity in the research community. Although different self-healing methodologies have been applied to functionalize the mechanism, much of the attention has been paid to the self-repair of damage (i.e. cracks) with no external intervention ( $\mathrm{Li}$ and Herbert, 2012). To assure robustness of this functionality and its intended purpose in real structures, self-healing will need to meet at least the following criteria: reliability, pervasively, stability, repeatability and economy.

The ability of concrete material to self-heal its own cracks is mainly associated with the formation of calcite and further C-S-H gels when no additional healing agents are used. Despite the effectiveness of both mechanisms in terms of crack plugging performance, changes in transport properties are more closely related to the formation of calcite, which is important for infrastructures that will be in contact with water or moisture during their service lives. The influence of self-healing on several transport properties of ECC has already been the topic of several studies. Lepech and Li (2009) studied the self-healing of microcracks in standard ECC mixtures where the extent was evaluated by changes in water permeability. According to this study, it was concluded that permeability of ECCs stabilized as a consequence of self-healing, and that damaged ECC specimens have similar water permeability coefficients as sound ones, provided that crack widths are kept under the threshold of $100 \mu \mathrm{m}$. Sahmaran et al. (2014) also reported 
substantial amounts of self-healing attainment in terms of chloride ion penetrability when precracked ECC specimens were allowed to self-heal their own damage. As one of the governing transport mechanisms, the diffusion of chloride ions in standard ECC (M45) mixtures ponded in $\mathrm{NaCl}$ solution was investigated by Sahmaran et al. (2007), who concluded that 30 days of exposure to salt solution led the microcracks in ECC to self-heal markedly, resulting in relatively low diffusion coefficients. Although all of the abovementioned studies are of great value and supply important information, permeability and diffusion may not be the governing mechanisms contributing to the transport of various media in cracked concrete, especially in cases where infrastructures are exposed to the drying actions of sun and wind. Under such conditions (dry and/or partially dry state), a dominant transport mechanism is more likely to be due to the capillary suction forces existing in the evacuated capillary cavities present in the matrix (Martys and Ferraris, 1997). When concrete material itself has a high capillarity, water and aggressive agents can more easily penetrate the material, accelerating rebar corrosion.

From a practical implementation point of view, the natural self-healing of tight cracks with no external interference is the simplest approach, despite the novelty of all the above-mentioned approaches. Therefore, many studies focusing on the effects of intrinsically well-controlled tight crack widths in ECCs and their relation to natural self-healing mechanisms have been conducted. These studies investigated the self-healing performance of ECCs under a number of commonly encountered environments, using pre-loaded specimens in the unloaded state. They concluded that durability, transport and mechanical properties can be recovered to a great extent under specific conditions (i.e. wet and/or partially wet environment) (Sahmaran et al., 2014; Yang et al., 2009; Lepech and Li, 2005; Sahmaran and Li, 2008; Li et al., 2007; Sahmaran and Li, 2009). However, upon unloading, cracks were almost half the width of those on loaded specimens (Yang et al., 2007; Zhou et al., 2009), which led to the overestimation of self-healing performance. This point is also important considering the so-called creeping effect, which causes further opening of existing cracks with extended service life. Therefore, in addition to the effects of environmental conditions, deterioration severity, age of pre-loading and so on, the influence of sustained loading on self-healing performance is of value, especially when the purpose is to obtain more conservative and realistic results. To account for the gap in the literature, a comprehensive study was previously undertaken to assess the effect of constant sustained loading and different environmental exposures on mechanical property recovery of ECCs (Ozbay et al., 2013). They concluded that even under 
constant sustained flexural loading, significant self-healing of microcracks took place. Although the sustained loading was monitored continuously, slight relaxations in stress values were inevitable under constant loading.

To date, little evidence has been reported concerning the capacity of more sizeable ECC beams for self-repair. These findings cannot be considered significant without the expansion of self-repair methods previously described, despite encouraging results from specimens on a more modest scale. Insufficient evidence to conclusively determine the dependability of the range of self-repair strategies indicates that additional studies on more samples are required. To ascertain the restoration of water tightness arising from self-repair in water-retaining vessels, Kishi et al. (2011) carried out a number of applied assessments. Concrete featuring mineral admixtures for self-report was produced in a ready-mix concrete factory and conveyed to a casting location in an agitator vehicle. The concrete was subsequently cast into square water-retaining vessels and after allowed to cure for several months. Next, $0.2 \mathrm{~mm}$ cracks were introduced and the vessels were filled with water. The extent of water seepage over time was evaluated to assess the degree of self-repair. The novel attributes of the self-repair concrete generated at the ready-mix factory were shown to meet construction application standards, and the mineral admixtures contained in the concrete allowed cracks to seal while minimising water leakage from the vessels. A three-month study on the selfrepair of ECC in a natural setting was carried out by Herbert and Li (2001), whereby samples were subjected to arbitrary and often severe environmental circumstances. It was reported that most of the cracks smaller than $20 \mu \mathrm{m}$ wide displayed self-repair upon visual inspection, while cracks of up to $150 \mu \mathrm{m}$ were also shown to be capable of repair in an artificial environment (Yang et al., 2009). Furthermore, ECC specimens in the natural setting were capable of regaining a sizeable part of their preliminary stiffness after three months, although this regain was lower than that of specimens examined under laboratory conditions. Despite the potential for ECC self-repair in a natural setting, it remains inferior to self-repair exhibited in the laboratory (Li and Herbert, 2012).

\subsection{Problem statement}

Robustness of self-healing concrete is dependent upon a myriad of variable parameters. These include the existence and width of cracks, environmental conditions such as temperature, humidity. These parameters are also dependent on the chemical composition of the supplementary cementitious materials. The ability of concrete to self-heal has been shown by researchers, yet 
robust characteristics of self-healing are not fully studied. Several studies have addressed the selfbehavior of pre-cracked ECC specimens without sustained mechanical loading, but no research work so far has been reported on the capability of crack healing under sustained mechanical loading as in the real field condition. Since the crack width of the ECC material is found to be significantly affected by the presence of sustained mechanical loading, it is also important to test the self-healing behavior of pre-cracked ECC specimens under sustained mechanical loading, which is of great importance for critical assessment of the performance of ECC under comprehensive conditions. As is common with many emerging materials, research related to material and durability properties precedes research on structural behavior. Because they are relatively new to the research community, self-healing behavior related to structural response of ECC is lacking.

Until these challenges are met, the widespread application of such self-healing materials remains disappointingly out of reach.

\subsection{Goals and objectives}

To quantify the robustness of self-healing, this research focused on the durability and healingability of microcracked ECCs incorporating different mineral admixtures with varying chemical compositions and several maturity levels, as well as, the effects of progressively increasing sustained loading and the effect of specimen size on the self-healing capability of ECCs.

To achieve the overall research goals, the following specific objectives are defined:

1. Understand the effects of environmental conditions (i.e. wet/dry), physical properties (i.e. crack width), and chemical compositions (i.e. available chemical species) on self-healing processes.

2. Study the durability in terms of sorptivity of pre-cracked and sound ECC specimens before and after exposure to environmental conditioning for crack healing, in order to better simulate actual field conditions and see how capillary suction is changed with the effect of self-healing. 
3. Observe the extent and rate of self-healing on rapid chloride permeability using mineral admixtures (MAs) with different chemical compositions (which can be decisive on selfhealing capability of the mixtures) in ECC mixtures.

4. Study the effects of progressively increasing sustained loading with different mineral admixture utilization on the self-healing capability of ECC mixtures.

5. Investigate the self-healing behavior of large-scale reinforced ECC beams under load influence by studying the mechanical performance of the pre-loaded beams before and after the healing.

\subsection{Guide to thesis}

Chapter 2 provides an introduction about failing concrete infrastructure, followed by a review on self-healing technologies along with the available test methods as well as their concrete mix design.

Chapter 3 deals with the effects of micro-cracking and type of cementitious materials on the durability performance of cementitious composites.

Chapter 4 examines the effect of self-healing on the different transport properties of cementitious composites incorporating different mineral admixtures.

Chapter 5 studies the self-healing capability of engineered cementitious composites under progressively increasing mechanical loading.

Chapter 6 observes the self-healing behavior of full-scale engineered cementitious composite beams with different shear span ratio.

Chapter 7 presents the conclusions and recommendations for future work. 


\section{Chapter 2 \\ Literature Review}

\subsection{Introduction}

This chapter presents an introduction about failing concrete infrastructure, followed by a review on durability concerns. The second part explains the self-healing technologies along with the cementitious composite materials design. The last part of this review emphasizes the use of supplementary cementitious materials (SCMs) and the test methods available to quantify the selfhealing.

\subsection{Failing concrete infrastructure}

Around the globe, nations struggle with ever increasing challenges of unsustainable development. The consequences of this struggle are often most visible in nations with rapidly expanding economies. Often, the backbone of such rapidly expanding economies is infrastructure development in the form of highways, airports, bridges, underground mass transit facilities, dams, wastewater treatment systems, marine structures and buildings, which support trade and encourage outside investment. Therefore, concrete material is, literally, one of the building blocks of economic development.

While essential for the development of economies, the construction and maintenance of these infrastructures often requires large volumes of Portland cement for use in concrete structures. The production of this material can result in tremendous public health impacts decades into the future. To illustrate, the production of one tonne of Portland cement releases $6.3 \mathrm{mg}$ of particles less than 10 microns in diameter (PM10) into the atmosphere (Marlowe, 2003). Numerous deaths annually have been attributed to atmospheric PM10 concentrations as low as $20 \mathrm{mg} / \mathrm{m}^{3}$ (Ostro, 1994). In addition to direct public health concerns, the production of Portland cement requires 4 GJ of energy per tonne, and releases into the atmosphere approximately 1 tonne $\mathrm{CO}_{2}$ per tonne of cement, representing in total $7 \%$ of worldwide $\mathrm{CO}_{2}$ emissions, and significantly contributing to global warming due to ozone depletion (Malhotra, 1998; Mehta, 1998). Furthermore, mining large quantities of raw materials such as limestone and clay, and coal for fuel in the cement kiln, often results in extensive deforestation and topsoil loss. The overall consequence is an inherently 
unsustainable system measured by economic, environmental, and social indicators. Sustainability requires the application of energy efficient materials with low impact on environment and improved durability. Many concrete structures are prone to extensive distress due to improper design, material selection, and construction practices for a given service environment. Therefore, the production of concrete is a serious concern for global environmental sustainability. In spite of these environmental problems, the global demand for concrete increases at a very rapid pace, and in less than one century, concrete has become the most widely used construction material in the world.

In most of the Design Codes, a service life time of $75+$ for concrete structures is now required in large public works (Alexander and Stanish, 2005). But experience has shown that under the combined effects of mechanical loads and environments, many infrastructures begin to deteriorate after only 20 or 30 years (Mehta and Burrows, 2001). The short service life of Portland cement concrete infrastructures has significant impacts due to materials production for repair and replacement of deteriorated infrastructure, along with fuel consumption and vehicle emissions from construction related traffic congestion. The poor durability of reinforced Portland cement concrete infrastructure associated with concrete cracking is one of the main reasons for this short service life of concrete infrastructure. Cracking is usually a result of various physical, chemical, and mechanical interactions between concrete and its environment. It may occur at different stages throughout the life of a structure. The formation of cracks coupled with a lack of crack width control in brittle concrete is primarily responsible for two damaging phenomena: reduction of the strength and stiffness of the concrete structure and acceleration of the ingress of aggressive ions which lead to other types of deterioration such as corrosion, alkali-silica reaction, freeze/thaw damage and sulphate attack resulting in further cracking and disintegration (Mindess et al., 2002). Therefore, durability is vitally important for all concrete structures, and it can be associated with the brittle nature of concrete.

It is an unfortunate fact that very large amounts of existing concrete structures worldwide are in a state of deterioration/distress (Vaysburd et al., 2004). Increased $\mathrm{CO}_{2}$ concentrations in the atmosphere are expected to further weaken the chemical stability of concrete in these structures (Engineers Canada, 2008). Maintaining and repairing structures has been a recurring need owed to the natural degradation of materials and infrastructure under the combined effects of mechanical 
loads and environmental factors. In the past, the practice was to replace old and deficient structures that had deteriorated or that were no longer suited to their original purpose with new ones. In today's nebulous economic climate and high cost of replacement, emphasis has been on repair of existing structures rather than new construction. While more and more 'effective' repair materials have been developed recently, concrete repair experiences continue to represent a 'mixed bag'.

Despite the best efforts of the repair industry, the failure rate for concrete repairs remains unacceptable. It has been estimated that almost half of all concrete repairs fail in the field (Mather and Warner, 2003). Concrete repairs are often perceived to lack both early age performance and long-term durability. Therefore, it is of utmost importance and challenging to develop effective,

economical, and durable (ultra-high performance) repair materials, which would fundamentally address the underlying concrete deterioration problems and protect the existing concrete from aggressive environments in the long term. In addition to focusing on effective and durable repair materials, challenges include the development of more environmentally benign materials and the effective utilization of by-products and waste materials in the production of repair materials.

\subsection{Concrete durability concerns}

Increased durability of reinforced concrete is typically associated with a dense concrete matrix, i.e. a very compact microstructure expected to lower permeability and reduce transport of corrosives to the steel (Oh et al., 2002; Beeldens and Vandewalle, 2001). This can be achieved with a well-graded particle size distribution (Hawang et al., 1996), fly ash and silica fume (Chang et al., 2001), or low water to cement (w/c) ratios (Mehta, 1986). These concepts, however, rely upon the concrete to remain uncracked within a structure throughout its expected lifetime and resist the transport of water, chloride ions, oxygen, etc. through its dense microstructure. In this presumed uncracked state, numerous concrete materials have shown promising durability in laboratory tests (Mora et al., 2003; Weiss and Shah, 2002).

In reality, however, reinforced concrete members crack due to both applied structural loading and shrinkage and thermal deformations, which are practically inevitable and often anticipated in restrained conditions (Mihashi and De Leite, 2004). These cracks provide pathways for the penetration of aggressive ions to cause concrete deterioration. Chlorides, oxygen, and carbonation agents can migrate through cracks and ultimately lead to corrosion of reinforcement. Although the 
uncracked concrete between adjacent cracks can be extremely dense and nearly impermeable, the presence of cracks results in a high overall permeability and unhindered access of corrosives (Hearn, 1999; Gerard et al., 1997; Bakker, 1988) to reinforcing steels through the concrete cover.

The early formation of shrinkage cracks coupled with a lack of crack width control in brittle concrete, is exacerbated through cyclic temperature changes along with wetting and drying cycles, leading to increased permeability of cracked concrete (Saadeghvaziri et al., 2005; Mihashi and De Leite, 2004). This then gives rise to elevated chloride concentrations, subsequent corrosion and volume expansion of reinforcing steel, and ultimately to disintegration of the matrix.

\section{3. “Crack free” concrete?}

Due to the inevitable occurrence of cracking within concrete resulting from a myriad of combined loads such as mechanical overloads, alkali silica reactions, sulfate reactions, temperature and moisture gradients or shrinkage stresses, and the subsequent deterioration and corrosion (Mindess et al., 2002), a number of initiatives have attempted to stem concrete pre-existing flaws. This approach is best exemplified by "Macrodefect-free materials" designed by a combination of polymer additives serving as 'lubricants' and externally applied pressure during processing for tight packing of cement grains (Birchall et al., 1981; Drabik and Slade, 2004; Mojumdar et al., 2004). Other attempts to reduce flaw sizes in concrete include optimizing grain size distributions (Hwang et al., 1996). However, such materials proved ineffective at eliminating cracks in field conditions due to increased brittleness despite their higher strength (Shah, 2000).

Within this thesis, a unique viewpoint of "crack free" concrete is taken. Cracks within concrete are primarily responsible for two damaging phenomena; increased concrete transport properties (Hearn, 1999; Gerard, 1997; Bakker, 1988) and reduction of concrete member load capacity. Therefore, virtually "crack free” concrete can be realized by resolving these two phenomena. Corrosion of reinforcing steel is the major cause of concrete deterioration worldwide, and the presence of cracking is the root cause of much of this deterioration (Nowak and Szerszen, 2003; Ciampoli, 2001). The autogenous healing of cracks to block transport of water and corrosives into the composite and down to reinforcing steel is achieved through formation of self-healing compounds such as calcite and hydration products within cracks (Ramm and Biscoping, 1998; Schiessl and Brauer, 1996). Second, self-healing of cracks can also regenerate a large portion of 
material load capacity and stiffness under mechanical loads (Sukhotskaya et al., 1983; Maugat and Gurusamy, 1987). Through formation of calcite and additional hydration products, over 80\% of damaged material stiffness has been shown to regenerate in preliminary studies. This research successfully counteracted both the increased transport properties and reduction in load capacity brought about by cracking through self-healing and thereby introducing virtually "crack free” concrete materials to extend the service lives of concrete structures.

Engineered Cementitious Composites (ECCs) developed over the past decades offer tensile strain hardening behavior due to controlled microcracks of widths less than $100 \mu \mathrm{m}$ (Li 2012). Strain hardening behavior (also referred to as ductility) may be regarded as high damage tolerance under mechanical loads and is provided via micromechanics-based material optimization (Li, 2003). Depending on the material design, tensile strain capacity in the range of $3-5 \%$ can easily be obtained with only $2 \%$ of fiber volume (Li et al., 2002). Ductile behavior as the result of multiple microcrack formation is considered a parameter with significant influence on durability (Yang et al., 2009).

Given the intrinsically well-controlled tight crack width in ECC, the effect of self-healing on the transport and residual mechanical properties of cracked ECC specimens has recently been investigated under a number of commonly encountered environments. Studies have been conducted using water permeation and submersion at room and high temperatures, wetting and drying cycles, chloride ponding and high alkaline environments (Sahmaran and Li, 2010; Lepech and Li, 2005; Sahmaran and Li, 2008). Typically carried out on pre-cracked specimens in the unloaded state, these studies show that mechanical and transport properties can be largely recovered in a wet environment, especially for ECC specimens preloaded to less than 1\% tensile strain at early ages. In addition to tight crack width, the relatively low water/binder ratio and significant amounts of unhydrated binders within the ECC mixture helped promote self-healing via continued hydration of cementitious materials. Calcite formation and continued pozzolanic reactions were also found to be important self-healing mechanisms (Li et al., 2007; Sahmaran and Li, 2009).

In addition to the impact of exposure conditions, pre-loading deformation level and pre-loading time on self-healing behavior, the influence of sustained mechanical loading should also be of 
interest due to the tendency of crack closure on unloading of ECC specimens. To account for crack closure on unloading, all crack width measurements were generally made in the unloaded state, and the self-healing capacity of ECC under different environments was also assessed in the unloaded state. Since crack width of ECC specimens under sustained load (close to or larger than $100 \mu \mathrm{m})$ is found to be almost double of the unloaded crack width, the performance achieved by damaged ECC without sustained loading may lead to an overestimation of the self-healing performance of pre-cracked ECC.

\subsubsection{Self-healing technologies}

Prior to exploring the self-healing phenomenon itself, identifying a set of desirable self-healing characteristics is helpful in narrowing the search for feasible and effective implementation technologies.

- Robust: Reliable, predictable self-healing in typical concrete structure environments,

- Pervasive: Ready for activation when and where needed (i.e. at the crack when cracking occurs),

- Stable: Remain active over the service life of a structure that may span decades,

- Repeatable: Ability to self-repair for multiple damage events, and

- Economical: Economically feasible for the highly cost-sensitive construction industry in which large volumes of materials are used daily.

Fortunately, concrete materials already possess a number of self-healing qualities through additional hydration (i.e. local activation, stability, quasi-repeatability, and low cost) due to a large amount (up to $25 \%$ depending on mix proportions) of unhydrated cement. While self-healing in some old concrete structures has been observed, more often than not, cracks remain unhealed. The poor robustness of this self-healing mechanism in normal concrete has limited any reliance upon such phenomena.

In light of these requirements and opportunities, the development of self-healing concrete materials has been extensively studied. One of the simplest approaches utilizes the intrinsic natural tendencies of continued hydration, pozzolanic, and carbonation processes when cracks in cementitious materials are exposed to water and carbon dioxide (in air), taking advantage of the 
existence of unhydrated cement grains and fly ash in a binder with low water/binder ratio. This approach can only work if the cracks are tight, $100 \mu \mathrm{m}$ or less. Ter Heide and Schlangen (2007) demonstrated this approach by continued hydration of damaged concrete specimens placed under water and subjected to a compressive load that closes the cracks. Li and co-workers demonstrated the ability of self-healing in ECC that tensile strain-hardens with crack widths self-controlled to below $50 \mu \mathrm{m}$. This tight crack width remains even when the material is loaded to several percent tensile strain (Lepech and Li, 2009). Evidence of self-sealing in ECC has been demonstrated by reduced permeability over time. Evidence of self-healing in the mechanical sense has been demonstrated by recovery of stiffness in predamaged specimens exposed to various environments (Li and Herbert, 2012).

Utilizing the intrinsic self-healing tendency of cementitious materials coupled with self-controlled tight crack width of ECC has proven to be a promising approach. Although no specific experiments have been conducted to determine the shelf life of this approach, it is likely that there would be a long shelf life since the intrinsic mechanisms of continuing hydration, pozzolanic reaction and carbonation in cementitious materials are known to be long lasting. This technique is highly pervasive since it utilizes unhydrated cement and fly ash, which are uniformly dispersed within the cementitious matrix. It has been found that the recovery of transport and mechanical properties can be $100 \%$ efficient depending on the preloading levels and exposure conditions (Sahmaran and $\mathrm{Li}, 2008)$. It has also been shown that this approach can be repeatable. A study concluded that there is a regain in stiffness for samples exposed to multiple damage and re-healing cycles. ECC self-healing has been demonstrated under continuous or intermittent water exposures, and at room and elevated temperatures (Yang et al., 2009) and at young and mature ages. In addition, ECC has been shown to self-heal under various adverse conditions, such as highly alkaline and chloride environments (Yang et al., 2011).

Aside from the limitations discussed above, a major obstacle to effective self-healing has been the lack of robustness, or low reliability. To form self-healing compounds or additional hydration products within cracks, tight crack width control has shown to be essential. Using reinforced concrete, such crack control is both difficult and unpredictable using equations like the GergelyLutz expression as proposed in earlier editions of the ACI building code (ACI Committee 318, 2014). Without a complete understanding of basic self-healing phenomena and the ability to 
reliability control concrete crack widths to spur self-healing processes under varying environmental conditions, robust self-healing materials can never reach widespread industry implementation.

\subsubsection{Realization of self-healing concrete}

Previous researchers have engaged in limited studies on the phenomenon of concrete self-healing, the formation of self-healing products, and the necessary conditions to experience self-healing in concrete materials. These studies have resulted in identifying three general criteria which are critical to exhibit self-healing; presence of specific chemical species, exposure to various environmental conditions, and small crack width. These are summarized as follows. In some instances, these findings are contradictory, as in the case of maximum allowable crack width in which some specify maximum crack widths of $10 \mu \mathrm{m}$ while others specify $300 \mu \mathrm{m}$ to exhibit selfhealing in various environmental conditions.

- Essential chemical species - Bicarbonate ions (HCO3-), Carbonate ions $\left(\mathrm{CO}_{3}{ }^{2-}\right)$, Free calcium ions $\left(\mathrm{Ca}^{2+}\right)$ (Jooss, 2001; Carola, 1999), Unhydrated cement (C3A) (Ter Heide, 2005; Jensen and Hensen, 2002), Free chloride ions ( $\left.\mathrm{Cl}^{-}\right)$(Jacobsen et al., 1996).

- Essential environmental exposure - Water (submerged) (Nanayakkara, 2003), environmental pH, wet-dry cycles (capillary suction) (Hannant and Keer, 1983), temperature above $80^{\circ} \mathrm{C}$ (Reinhardt and Martin, 2003), temperature above $300{ }^{\circ} \mathrm{C}$ (Farage et al., 2003).

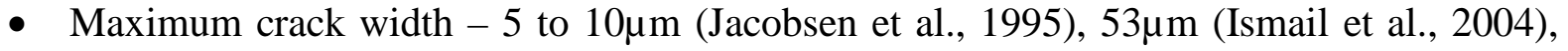

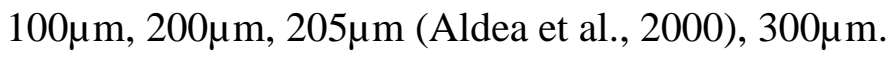

While the individual findings may differ, trends within these studies are clear. First, self-healing can occur in a variety of environmental conditions ranging from underwater to cyclic wet-dry exposures. Second, adequate concentrations of certain critical chemical species are essential to exhibit self-healing mechanisms. Finally, and potentially most important, is the ability to tightly control crack widths. While the other two criteria (environmental exposure and essential chemical species) are quite commonplace in many concrete applications, an inability to restrain crack widths below roughly $200 \mu \mathrm{m}$ prohibits the robust formation of self-healing products in most concretes. Using these above criteria, the development of materials which are designed to deliberately meet 
these requirements is possible. This set of material physical and chemical properties, and exposure conditions, may serve as a reference base towards systematic design of robust self-healing concrete.

\subsubsection{Preliminary self-healing tests}

To investigate the ability of cement-based composites with tightly controlled crack widths to exhibit self-healing, a series of preliminary self-healing experiments have recently been performed (Yang et al., 2005). These tests were conducted on a type of high performance fiber reinforced cementitious composite (HPFRCC) which exhibits both high tensile strain capacities (>3\%) along with self-controlled (without relying on steel reinforcement) tight crack widths $(<100 \mu m)$ even at high tensile strain levels (Figure 2.1). This is accomplished through the continuous formation of numerous microcracks under tensile load, until completely saturated (Figure 2.2). The use of such materials allowed for tight crack control to even the most stringent crack width criteria.

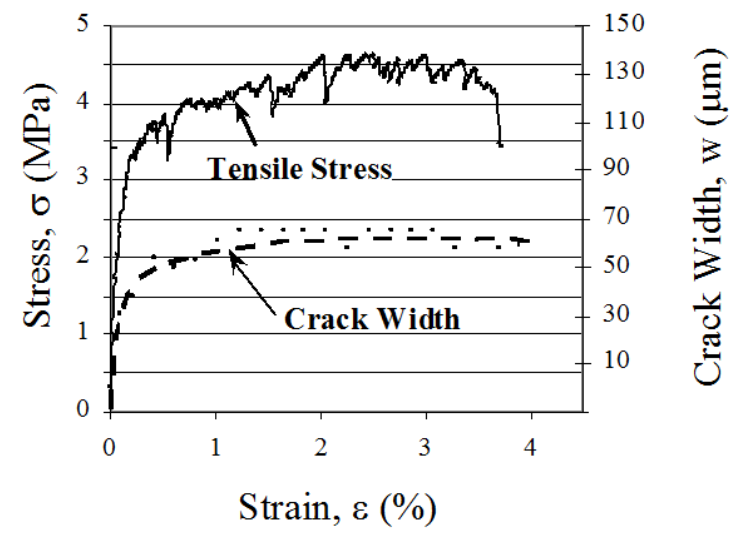

Figure 2.1: Uniaxial tensile response and crack width development of HPFRCC material

HPFRCC specimens have been first pre-cracked in tension to a pre-determined deformation level ranging from $0.3 \%$ to $3 \%$ strain, resulting in crack numbers between 9 and 90 , and maximum crack width kept between $40 \mu \mathrm{m}$ and $90 \mu \mathrm{m}$ (Table 2.1). The experimental program consisted of specimen submersion in $20^{\circ} \mathrm{C}$ water for 24 hours, $55^{\circ} \mathrm{C}$ oven drying for 22 hours, and cooling in laboratory air at $21 \pm 1^{\circ} \mathrm{C}$ for 2 hours. This was used to simulate cyclic outdoor environments such as sunshine and high temperatures.

Due to the tight crack widths, a significant amount of self-healing was observed. Regeneration of mechanical properties was most apparent among the most heavily damaged specimens, which 
experienced a $70 \%$ loss after initial loading and returned to over $90 \%$ of original stiffness through self-healing. Additionally, uniaxial tension tests of self-healed materials showed the formation of new cracks adjacent to previously healed cracks, thus verifying the potential for mechanical selfhealing (Figure 2.3). The characteristic of self-controlled tight crack width, and demonstrated selfhealing in such material provides confidence in the feasibility of designing 'crack-free' concrete materials with robust self-healing functionality.

Table 2.1: Number of cracks and maximum width in HPFRC specimen

\begin{tabular}{ccc}
\hline $\begin{array}{c}\text { Tensile Strain } \\
\text { \% }\end{array}$ & $\begin{array}{c}\text { Crack } \\
\text { Number }\end{array}$ & $\begin{array}{c}\text { Maximum Crack } \\
\text { Width } \boldsymbol{\mu m}\end{array}$ \\
\hline $\mathbf{3}$ & 80 & 83.25 \\
$\mathbf{2}$ & 48 & 46.4 \\
$\mathbf{1}$ & 15 & 67.6 \\
$\mathbf{0 . 5}$ & 12 & 89.7 \\
$\mathbf{0 . 3}$ & 9 & 43.15 \\
\hline
\end{tabular}

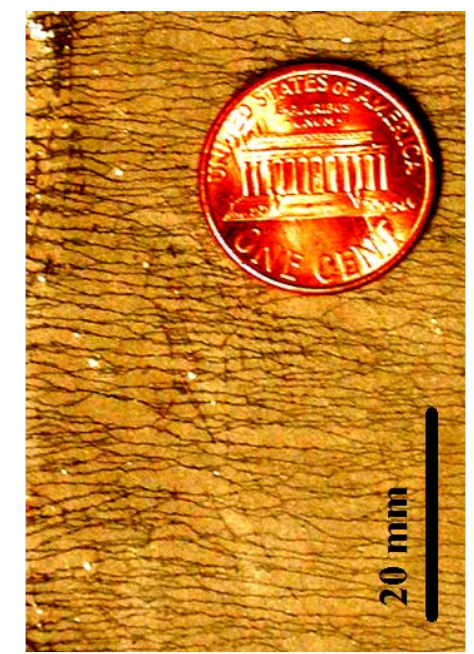

Figure 2.2: Crack formation in HPFRC (Li, 2003)

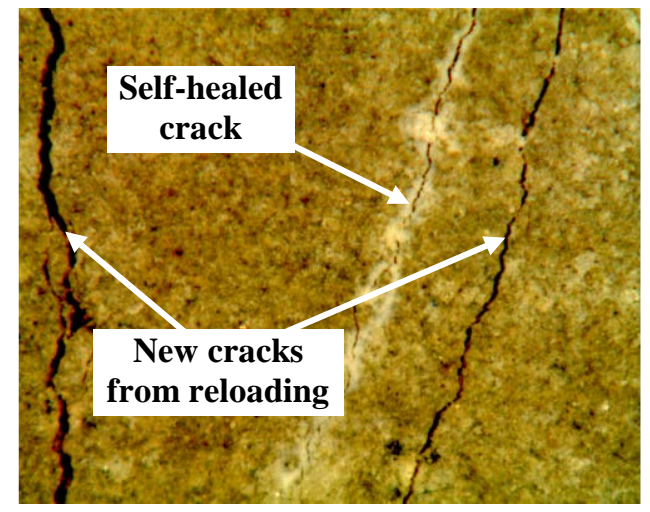

Figure 2.3: Cracks through virgin ECC material adjacent to a self-healed crack held tight by selfhealing material (Li et al., 2007) 


\subsection{Design and use of HPFRCC materials for self-healing}

In response to the poor tensile capabilities of traditional concrete materials, and the poor control of cracking in reinforced concrete members, HPFRCC materials have undergone significant experimental and theoretical development over the past 15 years (Li, 1993; Li, 1998; Li, 2003). While equivalent to concrete under compressive loads, these materials are far superior to concrete under tension. Showing a pseudo-strain hardening response after first cracking, HPFRCC composites offer both the mechanical properties and crack control behavior needed for realization of self-healing cementitious materials, as shown in Figure 2.2. Additionally, development of these composites has advanced to allow for material properties such as ductility and crack width to be intentionally designed by materials engineers (Weimann and Li, 2003).

These material design capabilities are based upon a toolbox of micromechanical models which guide the mode of cracking within these composites. Using these models Griffith type cracks are suppressed in favor of flat-type cracks which are highly conducive to deliberate crack width control. Thus, a high degree of material component tailoring can be achieved, allowing design of crack widths and crack spacing to promote self-healing processes (Figure 2.2).

At the heart of these design tools is the fiber bridging versus crack width curve, essentially a spring law describing the stress transferred across a crack as the width grows (Leung and Li, 1992; Lin and Li, 1997; Lin et al., 1999). Through manipulation of the fiber bonding and slip behaviors (Li and Stang, 1997; Leung and Li, 1991; Li et al., 1990; Li et al., 1991) which make up the stressstrain $(\sigma-\delta)$ relation, materials engineers can effectively design cementitious materials which intentionally meet specifications (i.e. crack width, etc.) for robust self-healing. Additionally, through tailoring of the matrix chemical makeup and transport properties, all other essential selfhealing criteria can be achieved while meeting basic material requirements such as compressive strength, tensile strength and ductility, and fresh material properties.

The critical $\sigma-\delta$ relationship is determined by fiber and fiber-matrix interface properties within the composite. High fiber modulus and strong interface bond are typically preferred for tight crack width control. However, high modulus fiber availability is limited and usually at high cost. Alternatively, adjusting interface properties has proven feasible to economically achieve controlled tight crack widths in cementitious composites. Approaches including fiber surface 
coating and interface transition zone densification will be used within the research to tightly control composite crack widths.

To guide the composite fiber-matrix interfacial design a theoretical model for maximum crack width will be used. It has been demonstrated that interfacial properties of certain polyvinyl alcohol fibers (PVA) can be tailored to exhibit a wide range via surface treatment with the objective of optimizing interface properties for crack width control (Rendon et al., 2001; Li et al., 2002; Kanda and $\mathrm{Li}, 1999)$. Crack widths below $100 \mu \mathrm{m}$ have been demonstrated. Crack widths below $50 \mu \mathrm{m}$, even at several percent tensile strain, are theoretically achievable. Ultimately, this design focuses on optimization of interface properties to control crack width for robust self-healing performance.

There are two distinct advantages to using HPFRCC materials to design self-healing materials. First is the capability of these materials to accommodate large imposed deformations. The cause of most concrete deterioration lies in the inability of concrete to deform without forming large localized cracks and thus leading to corrosion. This applies both to new construction, where deformations may be due to differential settlement, mechanical overload, or temperature gradients, along with repair construction which often shows extensive restrained shrinkage cracking. The use of ductile HPFRCC materials with controlled crack widths allows for self-healing in all of these varied construction scenarios. Monitoring of large HPFRCC structures in the field has shown that this tight crack width control translates from small laboratory specimens to large structures, independent of size (Rokugo, 2005). Second is the increasing acceptance of these materials within the construction community as an "equal" to concrete.

\subsection{Pozzolanic materials}

In document ACI 116R-90 (2005), the term 'pozzolan' is described as being 'a siliceous and aluminous or solely siliceous compound. When used as found - unmanipulated and unchanged it possesses almost no cementitious properties. However, if split into tiny pieces and introduced to water, it has a chemical response to calcium hydroxide when at a moderate heat. This reaction creates a material that does exhibit cementitious properties.' As explained by Hossain (2005, 2003, 2004, 2012a) and Hassan et al. (2012), a variety of different materials can be employed, from fly ash to volcanic ash, ground granulated blast furnace slag (usually referred to as just 'slag'), condensed silica fume, metakolin, rice husk ash, and more (Karahan et al., 2012). If a mineral 
compound is at once pozzolanic and cementitious, it may be utilised as a fractional substitute for something like Portland cement. In addition, if correctly utilized as a part of the cementitious compound, the aforementioned pozzolanic admixtures have the power to enhance the qualities of both recently poured and older cement. While a number of different auxiliary cementitious compounds are also used, this paper will concentrate on just two key varieties. They are fly ash and slag. As such, Chapters 2.4.1 and 2.4.2 will contain a basic outline of the background, qualities, and uses of fly ash and slag, within the concrete industry.

\subsubsection{Slag}

Slag has been used as partial replacement for Portland cement in concrete for more than 100 years (Glasser, 1991). As a by-product of the pig iron industry, slag is produced in large quantities in many areas around the world. In Canada, slag is most commonly used as base course for roads and other structures. However, in many other countries, most of the available slag is ground to approximately the same fineness as cement and then utilized as a partial replacement for Portland cement in concrete.

The composition of slag can vary considerably between production facilities. The presence of the major oxides is typically found to be within the following ranges: magnesium oxide (MgO), 0 to $21 \%$; aluminum oxide (Al2O3), 5 to $33 \%$; silicon dioxide (SiO2), 27 to $42 \%$; and calcium oxide (CaO), 30 to 50 \% (Taylor, 1997). Although the composition of slag is important, the method used in its production is perhaps more important as air-cooled slag has very limited, if any, cementing properties (Moranville-Regourd, 1998). However, if the slag is cooled rapidly from its liquid state at $1350-1550{ }^{\circ} \mathrm{C}$ down to about $800{ }^{\circ} \mathrm{C}$, crystallization of the material can be avoided and the resulting product often contains over 95\% glass which is a latent hydraulic cement (Taylor, 1997).

For any substantial reaction to occur between slag and water, an activator is required. High alkaline environments have proved to be suitable activators. Fortunately, the pore solution of cement paste is basically composed of alkaline hydroxides and as such, provides for an excellent activator. The use of slag in concrete tends to slow down the rate of hydration at early ages at room temperature. However, elevated temperatures help activate the slag and increase the rate of hydration (Roy, 1992). Therefore, slag can be used in steam-cured concrete. In addition, the damaging effects, such as reduced strength and increased permeability, of high early age temperature on concretes 
containing slag are less pronounced than when slag is not used (Neville, 1996). The reaction of a blend of cement and slag results in a higher percentage of calcium silicate hydrate (C-S-H) and less calcium hydroxide than plain cement (Neville, 1996). This alteration in the hydration products is attributable to the higher silicon content of the cement and slag blend when compared with plain cement. The change in the microstructure and the slower rate of hydration when slag is used typically yields denser and less permeable concretes.

In addition to lower permeability, concretes made with slag tend to have better resistance to chloride ion penetration than normal concretes. The freeze-thaw resistance of concrete made with slag is believed to be adequate and not adversely affected when compared with concrete made without slag (Neville, 1996). However, concretes made with slag generally suffer higher degree of deicing salt scaling than do reference concretes made without slag (Stark and Ludwig, 1997b). Consequently, ACI Committee 318 (2014) limits the maximum amount of slag to $50 \%$ of the total binder content if the concrete will be exposed to deicing chemicals. The total amount of slag and other supplementary cementitious materials is also limited to a maximum of $50 \%$ of the total binder content.

It is found that the contribution of optimized range of slag ECC mixture proportions to fiber dispersion outweigh the side effect of decreased potential for saturated multiple cracking, including a slight increase in matrix fracture toughness and fiber/matrix bond strength (Kim et al., 2007). The use of limestone powder and slag together as cement replacement in ECC mixtures exhibits high tensile strain capacity of 3.3\%, a tight crack width of $57 \mu \mathrm{m}$ and a moderate compressive strength of $38 \mathrm{MPa}$ (Zhou et al., 2009). Compressive strength development of slag concrete shows slower initial strength development for up to about 3 days, but will increase noticeably more than normal concrete after seven days. The rapid strength development of slag concrete makes it advantageous for structural applications (Alexander et al., 2003).

\subsubsection{Fly ash}

Fly ash is a waste product of the electric power industry. It is produced by the burning of pulverised coal. When the coal is burned, almost all of the carbon and the volatile compounds are eliminated. This isolates the mineral impurities of the coal (shale, quartz, feldspar, and clay). They eventually combine and merge together during suspension. The merged minerals are directed elsewhere by 
bag filters and electrostatic precipitators in the exhaust. Throughout this stage, the minerals are lowered in temperature and become solid. This produces round fly ash particles. The average particle is approximately 20 microns, but they can be as large as a hundred microns or as small as a single micron (Kosmatka and Panarese, 1988). The surface area can span anywhere between 200 and $700 \mathrm{~m} 2 / \mathrm{kg}$, but the average measurement is around $300-500 \mathrm{~m} 2 / \mathrm{kg}$. As outlined by the American Society for Testing and Materials, there are two key categories of fly ash (Table 2.2). These are Class F and Class C. While Class F is usually created using bituminous coals (ASTM C 618, 2015), Class C is created using subbituminous or lignite coals. Crucially, Class C fly ash is distinct for its ability to self-harden without the addition of cement. Furthermore, fly ash is mostly made up of iron, calcium, silica, and aluminum in a silicate glass structure. A number of additional minerals are present but only in very small amounts (magnesium, potassium, sodium, sulphur, and carbon). Currently, fly ash is the most commonly utilized mineral admixture in concrete.

Table 2.2: Specifications for fly ash (ASTM C 618, 2015)

\begin{tabular}{cc}
\hline Class of Ash & ASTM Specification \\
\hline Class C & $\mathrm{SiO}_{2}+\mathrm{Al}_{2} \mathrm{O}_{3}+\mathrm{Fe}_{2} \mathrm{O}_{3}>50 \%$ \\
Class $\mathbf{F}$ & $\mathrm{SiO}_{2}+\mathrm{Al}_{2} \mathrm{O}_{3}+\mathrm{Fe}_{2} \mathrm{O}_{3}>70 \%$
\end{tabular}

According to Shah and Peled (2003), fly ash actually lowers composite toughness, but improves fiber pull out. The combination of ECC compounds and fly ash (as a substitute for cement) leads to a decrease in crack tip strength and the fiber/matrix interfacial join. Nevertheless, the composite flexural stress might be lowered by $50 \%$ if a $70 \%$ proportion of fly ash is substituted for cement (Sahmaran and $\mathrm{Li}, 2009$ ). Plus, it is known that high amounts of fly ash usually lower matrix strength and the fibre/matrix join, in return for producing a high tensile strain ability (Wang and $\mathrm{Li}$, 2007). According to micromechanics evaluation, the boost to the fiber/matrix interface frictional join (within an ECC compound with high amounts of fly ash) is the reason for a tight crack width (Yang et al., 2007). It also enhances the overall strength and value of the compound by exhibiting increasingly saturated multiple cracking.

\subsection{Intrinsic self-healing capability of ECCs with different types of SCM}

\subsubsection{Mechanical properties}

2.6.1.1. Bending test 
There are several useful investigations into the recovery of mechanical capacities in the presence of bending checks. While explicit tension checks are thought to be the most effective way of determining the strain hardening capacity of a composite material (Qian and $\mathrm{Li}, 2008$ ), earlier research demonstrates a substantial link between deflection ability under bending and explicit tensile strain ability, particularly if the material is defined by genuine strain hardening qualities. According to one study (Qian et al., 2009), the main focus should be the retention of deflection abilities for strain hardening cementitious compounds (also known as SHCCs), as they are subjected to four point bending loading strains. The compounds employed in this research were produced using varying amounts of slag, limestone powder, and Class F fly ash. The SHCCs being investigated had a fairly high binder to water distribution. The researchers preconfigured beam specimens up to a $2.4 \mathrm{~mm}$ deflection capacity. They were then exposed to a constant stream of either (CA) continuous air or (CW) continuous wet, until they could be strained up until the point of failure under a four point bending load. As already discussed, moisture is a lot more powerful when it comes to retaining pre-test deflection values. For instance, even though beam specimens exposed to continuous moisture (CW) led to retention values of $65-105 \%$, the figures were only $40-62 \%$ for beam specimens exposed to continuous air. Substantial enhancements were demonstrated among original stiffness values after the addition of moisture, rather than air. On the other hand, the values for flexural toughness were dissimilar but not to a notable degree. Most importantly, the results indicate that constant moisture supports the creation of fresh deflection points and aids fibre bridging capacities.

After this investigation, Qian et al. (2008) conducted a supplementary study. The aim was to use the mixture to internally cure cementitious structures. The researchers created ECC compounds by incorporating a mixture of slag and limestone powder (alongside nanoclay particles). The specimens were exposed for a range of timeframes (14, 28, and 56 days). Following this, they were preconfigured beneath a four point bending load up to the point of a 1.5. $\mathrm{mm}$ deformation. The next step was to commence a number of curing cycles; direct air, direct moisture, a moist/dry rotation, and a 3\% CO2 exposure. The specimens were then preconfigured and loaded up until the point of failure to determine their capacity for self-recovery and the retention of mechanical qualities. The findings demonstrate that, in contrast with the reference tests, self-recovery among the ECC compounds was expressed in the form of increased deflection abilities. This was the case for all of the curing routines. In addition, the application of nanoclay was discovered to be valuable. It 
prompted a significant retention of deflection abilities, particularly when the mixtures were exposed to air. Moreover, a retention of stiffness was demonstrated among all the curing routines. In this case, the findings closely resembled those provided by earlier research (Yang et al., 2009). On the other hand, this retention of stiffness was not as substantial in mixtures that had been exposed for greater lengths of time. The unhydrated cementitious components were found to be reduced under these conditions.

The work of Ozbay et al. (2013) demonstrates the retention and self-recovery powers of ECCs when placed under a prolonged load. The researchers carried out experiments on EEC test samples that were 28 day olds. They tested specimens containing two distinct volumes of Class F fly ash (70\% ECC-2) and (55\% ECC-1). The weights were determined from the net volume of cementitious materials. The samples were preconfigured and loaded up until the point of $2.5 \mathrm{~mm}$ deformation, beneath a four point bending load strain, to test for crack vulnerability. Then, a prolonged load was incorporated at $60 \%$ of the total flexural strength of the ECC samples. Finally, both the unchanged and preconfigured samples were exposed to constant moisture or constant air routines. This was designed to test for self-recovery capacities, as they pertain to flexural values (flexural stiffness, mid-span beam deflection capacity, and flexural toughness). The results show that once the impact of a prolonged flexural load was considered, the eventual retention outcomes for the preconfigured samples dropped substantially, no matter what variety of curing routine they were exposed to. The samples exposed to moisture, but no prolonged load, exhibited a substantial improvement in deflection capacity and flexural stiffness (particularly when compared with samples exposed to constant air). The results are significant, because they demonstrate more modest self-recovery speeds than in investigations carried out on unloaded samples. This is primarily because the crack sizes are notably smaller after the load has been eliminated.

\subsubsection{Splitting tensile test}

A number of studies have turned to splitting tensile checks as a way to determine retention and self-recovery capacities, as they pertain to mechanical qualities. The researchers Ozbay et al. (2013) exposed round ECC samples (containing large amounts of fly ash) to pre-loading stresses, up until the point of $1.25 \mathrm{~mm}$ deformation, beneath a splitting tensile load. The samples contained the following volumes of fly ash; ECC-2 and ECC-1, in volumes of 70\% and 55\% of the net weight of cementitious materials, respectively. At the start of the research, they were all 28 days old. They 
were exposed to a wet/dry, a continuous wet, and a constant air rotation for a period of sixty days. Then, they were subjected to a second splitting tensile test as a way to determine the extent of their self-recovery capacities. The earlier pre-loading (at twenty-eight days) lowered the splitting tensile load bearing powers by $17 \%$ and $14 \%$ for ECC-2 and ECC-1, respectively. On the other hand, after a sixty-day period of moisture exposure, the figures lowered to $4 \%$ and $9 \%$, respectively (in comparison with the untouched samples). The findings demonstrate that the sixty-day period of wet/dry and continuous wet rotation were successful at substantially retaining load bearing powers, stiffness values, and deflection abilities among preconfigured ECCs. This is backed up by an elevated tensile ductility, a narrower crack size, and an increased volume of unhydrated cementitious components made accessible for additional hydration, within the ECC-2 compounds. Ultimately, the results show that ECC-2 is the stronger, more valuable material. This is the compound with the highest volume of fly ash.

\subsubsection{Transport properties}

\subsubsection{Rapid chloride permeability}

According to Misra et al. (1994), the rapid chloride permeability method is a fairly basic approach to verifying the quality of concrete (ASTM C 1202). However, the technique does not directly determine permeability or strength. It actually observes the electrical resistance exhibited by the compound. Nevertheless, as the method is so simple and quick to use, it has been employed in a number of valuable studies on the self-recovery abilities of ECCs. While the method provides some insights into the electrical conductivity of a concrete sample, its outcomes are thought to be indirectly associated with chloride ion strength performance and quality. It is influenced by a number of different variables, such as the chemical structure of the pore solution and the specific qualities of the pore solution (Shi, 2004).

A study conducted by Ozbay et al. (2013) evaluated the retention and self-recovery capacities of ECCs containing Class F fly ash. These precise amounts were determined according to the net weight of the cementitious materials. The researchers used the RCPT technique and investigated two distinct samples, each containing different amounts of fly ash (70\% ECC-and 55\% ECC-1). The experiments were carried out on samples that had been exposed to three distinct environmental routines for, initially, thirty days and then an additional sixty days. The three routines were 
continuous air, a wet/dry rotation, and continuous moisture. This occurred when they were 28 days old, but they were tested alongside the unchanged samples too. The samples were preconfigured with three splitting tensile deformation values $(0.75,1.00$, and $1.25 \mathrm{~mm})$. The proportional fluctuations among the RCPT outcomes, as they pertain to the 28 day findings, were contrasted for every preloaded value, exposure rotation, and compound variety. Across every one of the environmental routines, a number of dramatic decreases in chloride penetrability were observed among the ECC2 compounds. According to some researchers, continuous moisture and wet/dry rotations are more successful when it comes to obtaining RCPT insights, because they expand substantially once the varying pre-deformation loads have been added. They also argue that the eventual self-recovery substances that develop within the cracks are primarily made up of calcium carbonates and C-S-H gels. However, this research found that incorporating greater volumes of fly ash into ECC structures leads to expanded powers of retention and self-recovery. Within the later phases, this can be explained by the increased access to anhydrous cementitious substances for retention and self-recovery and the impact of substantially larger volumes of fly ash on the development of cracks with narrower dimensions.

The researchers Sahmaran et al. (2013) carried out an additional investigation that was designed to support the findings of the Ozbay et al. (2013) experiment. It was conducted in consideration of the fact that greater volumes of supplementary cementitious materials (also known as SCMs) indicate increased retention, as defined by the RCPT method. The study explored the impact of a number of distinct SCMs (Class-C fly ash/C_ECC, slag/S_ECC, and Class-F fly ash/F_ECC) on the self-recovery properties of ECCs. This was achieved by focusing primarily on RCPT values and outcomes. As a way to induce micro cracks, the samples being tested with the RCPT method were preconfigured beneath a splitting tensile load, until the following deformation points; $0.00 \mathrm{~mm}$ (untouched), $1.00 \mathrm{~mm}, 1.25 \mathrm{~mm}$, and eventual destruction. The amount of SCM utilised was restricted to a maximum of $70 \%$ of the net cementitious compound, according to total weight. The varying EEC compounds were exposed to continuous air, continuous moisture, and rotational thawing and freezing cycles.

Based on the self-recovery values produced by RCPT techniques, it is fair to say that the findings of the study indicate continuous moisture is the most influential type of exposure. This is the case both before and following the induction of micro crack destruction. The C_ECC and F_ECC 
samples produced outcomes that were substantially greater than the ones recorded after the initial sixty-day period, because they were exposed to a total of 300 thawing and freezing rotations. Crucially, the outcomes were greater no matter what the degree of pre-deformation. Ultimately, this is the equivalent of sixty extra days of curing exposure. On the other hand, among the S_ECC samples, the findings were smaller than those for the sixty-day period. To be precise, the condition led to smaller outcomes at the close of a 60+60-day timeframe. Such a result indicates that selfrecovery and retention - particularly those processes which occur within S_ECC compounds with micro crack damage - actively reverses degradation following the intense impact and influence of the prolonged thawing and freezing episodes.

The induction of thin cracks was discovered to be an important and essential part of stimulating self-recovery. The tolerable dimensions of these cracks (sizes that could still be recovered from) were close to 100, 50, and $301 \mathrm{~m}$ for S_ECC, C_ECC, and F_ECC samples, respectively. Within earlier research, calcite was believed to be the primary self-recovery substance among S_ECC samples. However, this research discovered that calcite and C-S-H gels play a significant part in self-recovery for C_ECC and F-ECC samples as well. The substantially greater self-recovery abilities of the S_ECC samples can be explained by the elevated PH conditions of the pore solution and the incorporation of slag within the ECCs. A number of aesthetic shifts among the cracks could also be seen, which is why retention and self-recovery capacities were further evaluated using EDX tools and SEM micrograph equipment. Ultimately, the study results demonstrate that eventual self-recovery capacities and products fluctuate, according to how much SCM is utilized.

\subsubsection{Absorption}

According to Ferraris and Martys (1997), within environments where the concrete compound is either completely or fractionally dry, movement can usually be attributed to the empty capillary pores in the matrix that produce subsequent capillary suction forces.

The work of Sahmaran and $\mathrm{Li}$ (2009) investigated the correlation between moisture sorptivity and micro crack development within an M45 ECC sample. The experiment was conducted on a compound created using a water resistant admixture and one with no such addition. The same researchers also carried out another investigation. This one was designed to contrast and compare sorptivity outcomes among ECC samples containing large amounts of fly ash. None of these 
samples contained a moisture resistant addition. The findings of both investigations demonstrate that the sorptivity values acquired from varying ECC samples reflect the maximum degrees of sorptivity for genuine, actual life structures. However, the self-recovery capacities (micro crack repair) were not properly considered throughout these experiments. These abilities could not have been tested, because sorptivity investigations are too brief to produce clear and accurate values of repair and retention. Nevertheless, the experiments indicate that the self-recovery of micro crack damage can usually be observed if a long enough testing period is adhered to. This is because selfrecovery speeds up when damage is introduced to wet conditions.

The work of Zhang et al. (2014) explores the self-recovery capacities of both undamaged and degraded ECCs. All of the tested compounds contained varying volumes of fly ash and were assessed by recording their sorptivity values on a number of different days. The results of the study demonstrate that all of the ECC compounds exhibited substantial decreases in moisture sorptivity after prolonged curing exposure. This is due to self-recovery developing inside the micro cracks, as a consequence of the secondary hydration of the fly ash and the persistent hydration of cement particles.

\subsection{Review Conclusion}

There are lots of previous research studies on the impact of SCMs, as they pertain to the mechanical and strength based qualities of cementitious components. However, there is a deficit of research focus on the impact of SCM types on the self-healing abilities of ECCs. Also, there is almost no information on the effects of pre-loading and re-loading deformation levels or exposure to distinct environment configurations on the self-healing.

To rectify this deficit, this research was planned and it was a timely initiative. It examined the selfrecovery ability of ECC that contained a range of four different mineral admixtures. These four distinct varieties were as follows; high calcium fly ash, slag, and low calcium fly ash. The study focused on the strength, transport, and mechanical properties of micro-cracked ECC samples, after curing exposures of 7, 28, and 90 day periods. It also examined the impact of sustained loads on ECC samples that had first been exposed for 180 days. Moreover, the self-healing behavior of large scale ECC reinforced beams under flexure load was investigated. The quality and speed of 
self-recovery was evaluated according to the mechanical, transport and flexural properties of the virgin, pre-loaded and re-loaded beams. 


\section{Chapter 3}

\section{Effects of Microcracking and Type of Cementitious Materials on the Mechanical and Durability Performance of Cementitious Composites}

\subsection{Introduction}

This chapter presents the relationship among the micro-cracking and chloride ion permeability of ECC mixtures that contain different supplementary cementitious materials along with the effects of different SCM types on some of the mechanical properties. Four SCMs, representing a wide range of compositions, are used in the study. The splitting tensile deformations are introduced to generate micro-cracks in specimens, where cylindrical specimens are pre-loaded up to $80 \%$ of their splitting deformation capacities. Rapid chloride permeability test (RCPT) is used to assess the effect of microcracking and SCM type on the chloride ion permeability of concrete. This chapter also focuses on the frost durability of sound and microcracked ECCs incorporating different mineral admixtures with varying chemical compositions, with performance evaluated based on sorptivity measurements repeated after specific numbers of F/T cycles. Moreover, fresh and hardened air-void parameters of different ECC mixtures are investigated and compared.

\subsection{Experimental Program}

\subsubsection{Materials and Mixture Proportions}

ECC mixtures with four different mineral admixtures (MAs) were produced. MA types were low lime fly ash (FA_L, [ECC_L]), medium lime fly ash (FA_M, [ECC_M]), high lime fly ash (FA_H, [ECC_H]) and ground granulated blast furnace slag (Slag, [ECC_S]). Although both FA_L and FA_M were Class-F type fly ash in accordance with ASTM C 618, nomenclature of the mixtures was determined depending on their lime content for clearance. In addition to the different MA types, mixtures included general use CEM I 42.5R ordinary Portland cement (PC), silica sand with $400 \mu \mathrm{m}$ maximum aggregate size and $0.3 \%$ of water absorption capacity, water, high range water reducing admixture (HRWRA) and poly-vinyl alcohol (PVA) fibers with a diameter of $39 \mu \mathrm{m}$, nominal tensile strength of $1610 \mathrm{MPa}$ and specific gravity of 1.3. Chemical and physical properties 
of PC and MAs and particle size distributions of solid ingredients are provided in Table 3.1 and Figure 3.1, respectively.

Table 3.1: Chemical and physical properties of PC and different SCM types

\begin{tabular}{lccccc}
\hline Chemical Composition, \% & PC & FA_L & FA_M & FA_H & Slag \\
\hline $\mathrm{SiO}_{2}$ & 20.77 & 57.01 & 54.94 & 41.8 & 35.06 \\
$\mathrm{Al}_{2} \mathrm{O}_{3}$ & 5.55 & 20.97 & 22.33 & 18.78 & 10.4 \\
$\mathrm{Fe}_{2} \mathrm{O}_{3}(\mathrm{~T})$ & 3.35 & 4.15 & 3.9 & 6.44 & 0.79 \\
$\mathrm{MnO}$ & - & 0.032 & 0.045 & 0.019 & 0.387 \\
$\mathrm{MgO}$ & 2.49 & 1.76 & 1.24 & 4.66 & 12.24 \\
$\mathrm{CaO}$ & 61.4 & 9.78 & 11.89 & 21.77 & 38.34 \\
$\mathrm{Na} 2 \mathrm{O}$ & 0.19 & 2.23 & 2.36 & 1.74 & 0.12 \\
$\mathrm{~K}_{2} \mathrm{O}$ & 0.77 & 1.53 & 0.79 & 0.63 & 0.37 \\
$\mathrm{TiO}_{2}$ & - & 0.68 & 0.68 & 1.5 & 1.08 \\
Loss on Ignition & 2.2 & 1.25 & 1.77 & 0.68 & 1.12 \\
Total & & 99.48 & 100.1 & 98.88 & 97.66 \\
\hline Physical Properties & & & & & \\
\hline Specific Gravity & 3.06 & 2.02 & 2.59 & 2.61 & 2.87 \\
Blaine Fineness (m & & & & \\
& 325 & 290 & 306 & 315 & 430 \\
& & & & & \\
\hline
\end{tabular}

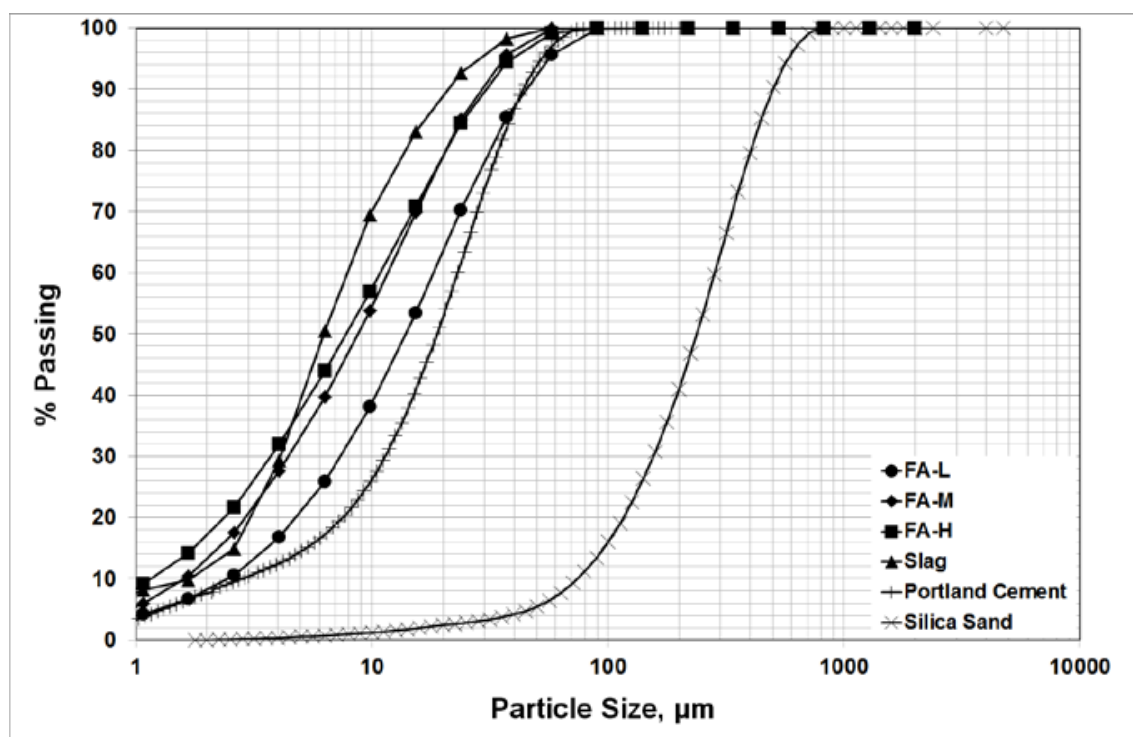

Figure 3.1: Particle size distributions of solid ingredients

Table 3.2: Mixture proportions and compressive strength results of ECC mixtures

\begin{tabular}{|c|c|c|c|c|c|c|c|c|c|c|}
\hline \multirow{2}{*}{ Mix ID. } & \multirow{2}{*}{ PC } & \multirow{2}{*}{ W/CM } & \multirow{2}{*}{ Sand } & \multirow{2}{*}{$\mathrm{FA} / \mathrm{PC}$} & \multirow{2}{*}{$\mathrm{S} / \mathrm{PC}$} & \multirow{2}{*}{$\begin{array}{l}\text { HRWR } \\
\left(\mathrm{kg} / \mathrm{m}^{3}\right)\end{array}$} & \multirow{2}{*}{$\begin{array}{c}\text { Fiber } \\
\left(\mathrm{kg} / \mathrm{m}^{3}\right)\end{array}$} & \multicolumn{3}{|c|}{ Compressive Strength, MPa } \\
\hline & & & & & & & & $7 \mathrm{~d}$. & $28 \mathrm{~d}$. & $90 \mathrm{~d}$. \\
\hline ECC_L & 1.0 & 0.27 & 0.80 & 1.2 & - & 3.9 & 26 & 32.9 & 55.5 & 66.2 \\
\hline ECC_M & 1.0 & 0.27 & 0.80 & 1.2 & - & 4.2 & 26 & 50.9 & 54.7 & 69.5 \\
\hline ECC_H & 1.0 & 0.27 & 0.80 & 1.2 & - & 4.6 & 26 & 55.1 & 58.7 & 61.9 \\
\hline ECC_S & 1.0 & 0.27 & 0.80 & - & 1.2 & 5.3 & 26 & 64.7 & 77.2 & 81.5 \\
\hline
\end{tabular}


Table 3.2 presents details of mixture proportions, which show that ECCs were produced with identical water to cementitious material ratio (W/CM) of 0.27 and mineral admixture to Portland cement ratio (MA/PC) of 1.2, by mass.

\subsubsection{Specimen preparation, pre-cracking and testing}

Mechanical properties of ECC mixtures were investigated through compressive strength, flexural strength and mid-span beam deflection evaluations. All the tests related to mechanical properties were performed at the end of 7, 28 and 90 days. For compressive strength tests, ASTM C 39 procedure was followed and final results were calculated by taking the average of values obtained from at least six different cubic specimens with the dimension of $50 \mathrm{~mm}$. For the determination of flexural parameters, prism specimens measuring 360×50x75 mm were prepared and tested under four-point bending loading. The results of at least six different prisms were averaged to obtain the final values. Specimens to be used for the evaluation of mechanical properties were stored in plastic bags at $23 \pm 2{ }^{\circ} \mathrm{C}$ and $95 \pm 5 \% \mathrm{RH}$ until the predetermined testing ages after one-day curing in the molds at $23 \pm 2{ }^{\circ} \mathrm{C}$ and $50 \pm 5 \% \mathrm{RH}$.

To assess chloride ion penetrability resistance of ECC mixtures, rapid chloride permeability test (RCPT) was performed in accordance with ASTM C 1202. After one-day curing in laboratory conditions at $23 \pm 2{ }^{\circ} \mathrm{C}$ and $50 \pm 5 \% \mathrm{RH}, \varnothing 100 \times 200 \mathrm{~mm}$ cylindrical specimens were removed from the molds and from each specimen three $\varnothing 100 \times 50 \mathrm{~mm}$ pieces were extracted from the middle portions to be used in RCPTs. Specimens were then kept in plastic bags at $23 \pm 2{ }^{\circ} \mathrm{C}$ and $95 \pm 5 \%$ $\mathrm{RH}$ until the end of 7, 28 and 90 days. In order to create microcracks and observe the effects of cracking on chloride ion penetrability results, $\varnothing 100 \times 50 \mathrm{~mm}$ ECC specimens were pre-loaded up to $80 \%$ of their splitting tensile deformation capacities at the end of 7, 28 and 90 days by using closed-loop controlled material test system at a loading rate of $0.005 \mathrm{~mm} / \mathrm{s}$ and comparison has been made between RCPT results of sound and pre-loaded specimens.

To test water sorptivity after exposure to cyclic freezing and thawing, beam specimens measuring $360 \times 75 \times 50 \mathrm{~mm}$ (length $\times$ depth $\times$ width) were prepared from ECC mixtures. Casting was completed in a single layer without any compaction, and all specimens were removed from their molds after 24 hours of curing in a laboratory medium at $23 \pm 2{ }^{\circ} \mathrm{C}, 50 \pm 5 \% \mathrm{RH}$. After 13 days of moist curing in lime-saturated water at $23 \pm 2{ }^{\circ} \mathrm{C}$, specimens were removed from the water and kept in a 
laboratory medium for one additional day. On the 14th day, some of the beam specimens were pre-loaded up to $80 \%$ of their mid-span beam deflection capacities under four-point bending loading. This common level of pre-loading was selected for all mixtures since ECC specimens produced with different MAs were highly likely to result in varying mid-span beam deflection results. In addition, some (sound) specimens were kept with no applied pre-loading, to be used as control specimens. As with the mechanical pre-loading under bending, $75 \times 75 \times 50 \mathrm{~mm}$ (length $\times$ depth $\times$ width) prisms were cut out of the central portion of each beam specimen for water sorptivity testing (Figure 3.2). The procedure described in ASTM C 1585 standard was used for sorptivity tests. Five pre-loaded specimens from each mixture were tested along with two sound specimens with no pre-loading as comparison. Special care was taken to ensure that different crack numbers were introduced to the pre-loaded specimens. Specimens were dried out in an oven at $50 \pm 5{ }^{\circ} \mathrm{C}$ for three days to confirm a constant weight before testing. For sorptivity testing, specimens were plunged into water up to $3-5 \mathrm{~mm}$, as seen in Figure 3.2. The lateral sides of the specimens were carefully sealed with a silicon coating to assure unidirectional water movement into the specimens and to reduce water evaporation from the sides. Changes in mass of ECC prism specimens left to absorb water through capillary action were recorded at time intervals of 1, 2, 3, 4, 6, 8, 12, 16, 20, 25, 36, 49, 64, 81, 120 and 360 minutes. The rate of absorption (mm), defined as the change in mass (g) divided by the cross sectional area of the test specimen $\left(\mathrm{mm}^{2}\right)$ and the density of water at the recorded temperature $\left(\mathrm{g} / \mathrm{mm}^{3}\right)$, was plotted against square root of time $\left(\min ^{1 / 2}\right)$. The slopes of the obtained lines are regarded as the sorptivity index $\left(\mathrm{S}_{\mathrm{o}}\right)$ of ECCs during the six hours of initial testing. For all specimens, this slope was determined by using least-squares, linear regression analysis of the plot of absorption rate versus square root of time. After initial sorptivity measurements were recorded, specimens were subjected to $300 \mathrm{~F} / \mathrm{T}$ cycles according to ASTM C 666 Procedure A, the specimens were kept in fully saturated conditions with temperatures cycling between -17.8 and $4.4{ }^{\circ} \mathrm{C}$. Sorptivity measurements were repeated after each of the $50 \mathrm{~F} / \mathrm{T}$ cycles; before the tests, the previously-mentioned drying method was applied to empty capillary pores. Each F/T cycle took around five hours, with each five cycles taking about one day of exposure. The Modified Point Count Method, in accordance with ASTM C 457, was also used for the characterization of air-void parameters on polished specimens of different ECC mixtures. Measurements of the 14-day-old hardened ECCs were made under a microscope by scanning along a series of transverse lines. 


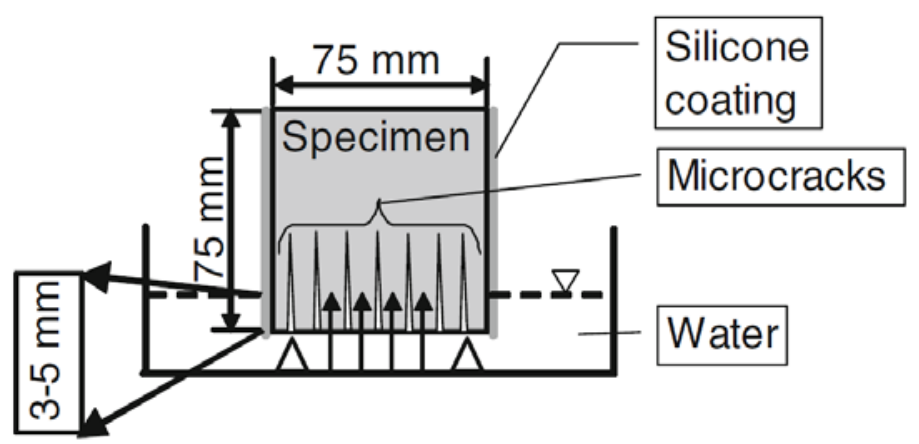

Figure 3.2: Schematic representation of water sorptivity test setup and cut-off prism specimen

\subsection{Results and discussion}

\subsubsection{Compressive strength}

The compressive strength test results of ECC specimens were shown in Table 3.2. As seen from the table, all of the results showed increasing trend until the end of 90 days regardless of the type of SCM used in the mixtures although increasing trend was slightly less pronounced between 28 and 90 days of age in general. This behavior of all ECC specimens produced in this study can be seen more visibly in Figure 3.3 where compressive strength values were plotted against age. As seen from the figure, the curves between 7 and 28 days of age are steeper compared to the curves between 28 and 90 days of age in general showing high compressive strength attainment between this time period which is most probably in relation with the abundant existence of unhydrated cementitious particles at early ages.

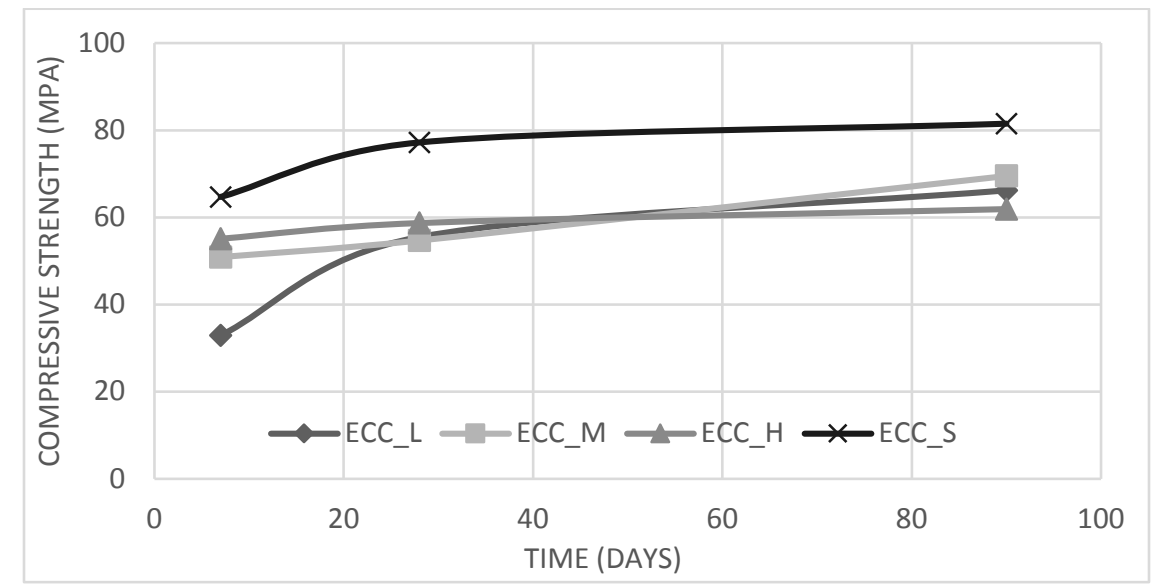

Figure 3.3: Compressive strength results with respect to different curing ages

When a careful inspection is made it can be stated that ECC specimens incorporating slag showed the highest compressive strength values at the end of each specified age. For example, while 
ECC_S specimens showed 64.7 MPa of compressive strength after 7 days, the same value was 32.9, 50.9 and 55.1 MPa for ECC_L, ECC_M and ECC_H specimens, respectively. This behavior of ECC_S specimens can be more understandable by controlling the chemical and physical properties of the SCMs presented in Table 3.1. As Table 3.1 makes it clear, CaO, which is the main ingredient for enhanced cementing behavior, is relatively high in the case of slag along with the substantially high amount of $\mathrm{SiO}_{2}$ which is mostly responsible for the pozzolanic reactions at later ages. This therefore implies that C-S-H gel formation could be expected to be accelerated in the case of ECC_S specimens, which would significantly contribute to higher compressive strength results. Another plausible explanation for the higher compressive strength results monitored in ECC_S specimens could be the filler effect. As seen from Table 3.1, with $430 \mathrm{~m}^{2} / \mathrm{kg}$ of specific surface area, slag has the highest fineness compared to the other SCMs. This leads slag particles to better fill the spaces among cement grains leading to improved particle size distribution and more compact microstructure. Moreover, high surface area of slag provides more nucleating sites and $\mathrm{OH}-$ ions as well as alkalis into the pore fluid ( $\mathrm{Li}$ and Zhao, 2003).

When fly ash incorporating specimens are considered it can be seen that ECC_L specimens showed the lowest compressive strength while ECC_H specimens showed the highest results at the end of 7 days. When curing time was extended to 90 days however an opposite situation was observed. This also clarifies the importance of $\mathrm{CaO}$ amount on the cementing behavior and $\mathrm{SiO}_{2}$ amount on further pozzolanic reaction considerations when a suitable SCM is to be preferred.

\subsubsection{Flexural parameters}

Although direct tensile test is considered to be the most accurate method to confirm the strainhardening behavior of a composite, implementation of this test method is not always easy due to several reasons. Previous studies demonstrated that deflection capacity under bending can be correlated with the tensile strain capacity when the material is truly strain hardening (Qian and Li, 2008). Therefore, in this study, it was decided to use the four-point bending test to investigate the flexural strength and ductility of ECC mixtures. Flexural parameters including both flexural strength and deformation values were tabulated in Table 3.3.

When the data in Table 3.3 are evaluated, one can see that all flexural strength results varied between the values of 7.5 and 11.9 MPa depending on age and SCM type used in the mixtures. 
Results showed increasing trend until the end of 90 days of age irrespective of the mixture type although increments were less pronounced between the ages of 28 and 90 days. In comparison to the changes observed in compressive strength results, changes in flexural strength results were minimal so that between the ages of 7 and 90 days there was 16.8 MPa increase in compressive strength results of ECC_S specimens while the same value was only 1.1 MPa for flexural strength results. The most probable reason for this trend may be attributed to the fact that flexural strength is governed by more complex material properties, such as tensile first cracking strength, ultimate tensile strength and tensile strain capacity, particularly in the case of strain hardening cementitious materials (Qian et al., 2009).

Table 3.3: Flexural parameters of ECC specimens

\begin{tabular}{lcccccc}
\hline \multirow{2}{*}{ Mix ID. } & \multicolumn{2}{c}{ Flexural Strength (MPa) } & \multicolumn{3}{c}{ Flexural Deformation (mm) } \\
& 7d & 28d & 90d & 7d & 28d & 90d \\
\hline ECC_L & 8.3 & 10.2 & 10.7 & 5.3 & 4.3 & 3.9 \\
ECC_M & 9.1 & 9.9 & 10.6 & 4.1 & 3.8 & 2.8 \\
ECC_H & 9.8 & 11.6 & 11.7 & 4.0 & 2.7 & 2.6 \\
ECC_S & 10.8 & 11.5 & 11.9 & 2.2 & 2.0 & 1.7 \\
\hline
\end{tabular}

Flexural deformation values which are the reflections of the ductility of ECC materials were shown in Table 3.3 as well. As seen from the table, for the first 7 days of curing, flexural deformation values ranged between 2.2 and $5.6 \mathrm{~mm}$ depending on the type of SCM used in the mixtures. When the curing time prolonged, there was a clear decrease in the flexural deformation results regardless of the mixture type. For example, 7-day flexural deformation value of ECC_L specimens was 5.3 $\mathrm{mm}$ while the same value for 90 days of age was $3.9 \mathrm{~mm}$. Decreasing trend in the results was associated with the continuous evolution of fiber/matrix interface properties with time. Among all different mixture types, ECC_S specimens showed the lowest flexural deformation values at the end of all specified days. Reduced ductility observed especially in the case of ECC_S and ECC_H mixtures was also realized in terms of final crack width measurements. For example, after the application of pre-loading to the specimens, observed crack widths of ECC_S and ECC_H mixtures were found to be ranging between 100-150 $\mu$ m levels in general while the same values were lower than $80 \mu \mathrm{m}$ level for the other mixtures showing that utilization of slag and high calcium fly ash in the mixtures triggered the formation of cracks with wider widths which resulted in localized failures and reduced ductility (Figure 3.4). When the flexural deformation capacity of normal concrete and conventional fiber reinforced concrete is considered to be around $0.5 \mathrm{~mm}$ 
level, even the lowest deformation value obtained from ECC_S specimens at the end of 90 days is several times that of normal concrete which is a remarkable achievement.

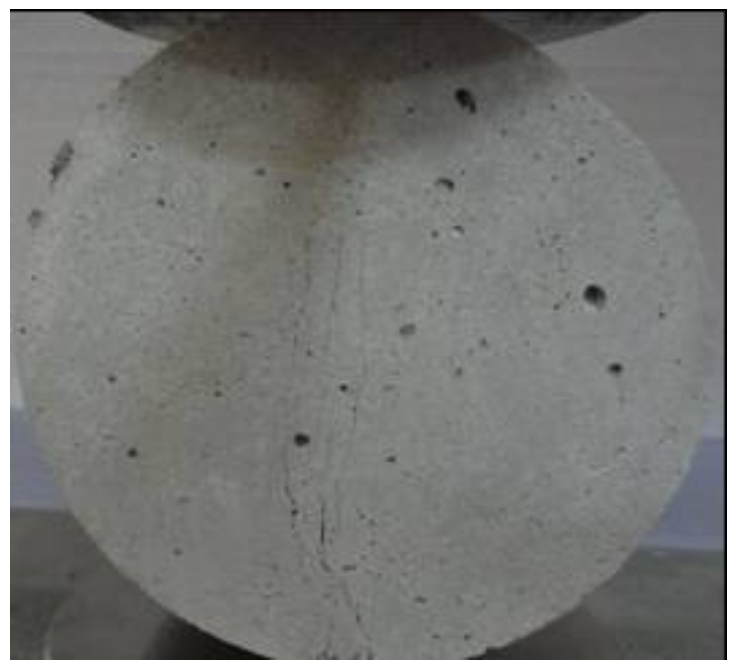

Figure 3.4: Formation of multiple microcracks over an ECC cylinder specimen upon exposure to splitting-tension pre-loading

\subsubsection{Rapid chloride permeability test (RCPT)}

Rapid chloride permeability test results of ECC mixtures incorporating different SCMs were shown in Table 3.4.

As it is obvious in the table, results showed marked variations depending on the age and type of SCM used. Chloride ion penetrability results showed significant reductions as the initial curing time of specimens was extended. For example, in the case of ECC_L specimens, chloride ion penetrability value obtained after 7 days was 7169 coulombs in average while this value decreased to 375 coulomb level after 90 days of curing. This behavior observed in the case of ECC_L specimens held true for the rest of ECC specimens as well. Reason for the decreased results with time is correlated with the enhancements in hydration and pozzolanic reactions which lead denser microstructure and less permeable matrices with time.

Table 3.4: Rapid chloride permeability test results of ECC specimens

\begin{tabular}{lcccccc}
\hline \multirow{2}{*}{ Mix ID. } & \multicolumn{3}{c}{ Sound Specimen } & \multicolumn{3}{c}{ Pre-loaded Specimen } \\
& 7d & $28 \mathrm{~d}$ & $90 \mathrm{~d}$ & $7 \mathrm{~d}$ & 28d & 90d \\
\hline ECC_L & 7169 & 1615 & 375 & 8318 & 3297 & 1308 \\
ECC_M & 5905 & 1947 & 430 & 7333 & 2757 & 1309 \\
ECC_H & 4863 & 956 & 859 & 6289 & 1654 & 1511 \\
ECC_S & 6397 & 2283 & 1195 & 7888 & 3549 & 3686 \\
\hline
\end{tabular}


Another point that needs to be stressed out is that pre-loading caused significant increases in chloride ion penetrability results regardless of age and SCM type. For instance, 7-day average RCPT results of ECC_H specimens increased from 6397 coulombs up to 7888 coulombs when the specimens were pre-loaded up to $80 \%$ of their splitting tensile deformation capacities. This behavior of specimens was much more pronounced when they were further aged. When ECC_S specimens are considered as an example, it can be seen that pre-loading caused $23 \%$ of increase in chloride ion penetrability of 7-day-old specimens although increase in RCPT results escalated up to $55 \%$ and $208 \%$ of the initial values in the case of 28-day-old and 90-day-old specimens, respectively. This behavior observed in the case of further aged specimens could be attributed to the enhanced matrix maturity of mixtures provided by the extended curing time which causes increased matrix fracture toughness values triggering the formation of localized cracks with wider widths instead of steady-state multiple microcracking (Wang and Li, 2007). Although, the escalations in chloride ion penetrability results with prolonged curing times seem alarming it should be kept in mind that all of the 90-day-old ECC specimens stayed in the low chloride ion permeability level as prescribed by ASTM C 1202 after the application of pre-loading except with ECC_S specimens whose RCPT results increased to moderate level upon the application of preloading. Overall these findings suggest that different from water permeability results (Lepech and $\mathrm{Li}$, 2005), chloride ion permeability of ECC specimens is substantially influenced by the application of pre-loading and presence of micro-cracking.

The air void characteristics of 14-day-old ECC specimens are shown in Table 3.5; each value is an average of two specimens. According to the table, it is evident that fresh air content of ECCs, calculated according to ASTM C 231, changed between 6.1 and 7.2\% depending on MA type. Despite the fact that fresh air contents of different ECCs are higher than in conventional concrete mixtures (due, most probably, to the lack of coarse aggregates, as well as the high viscosity of ECC matrices and the presence of PVA fiber), these values were found to be generally adequate for F/T durability (ACI Committee 345, 2011). One notable point related to Table 3.5: ECC_S specimens had the highest amount of fresh air content while ECC_L specimens had the lowest, although the difference was not drastic. This finding should be explored with particular attention to the viscosity of different mixtures. According to Yang et al. (2009), plastic viscosity of ECC mixtures is affected by the changes in W/CM ratio to a great extent, followed by the changes in HRWRA/CM ratio to a lesser extent. Given the fact that all ECC mixtures were produced with the 
same W/CM ratio of 0.27 , it can be deduced that higher fresh air contents in specimens with slag were a result of increased HRWRA amounts in the mixtures, which were necessary for the attainment of adequate fresh properties (Table 3.2). Relatively higher fineness and increased viscosity of ECC_S systems over those with fly ash likely prevented some air bubbles from rising to the surface of the specimen during placement, leading to higher fresh air contents.

Table 3.5: Air-void characteristics of different ECCs

\begin{tabular}{l|llll}
\hline Properties & ECC_L & ECC_M & ECC_H & ECC_S \\
\hline Fresh air content (\%) & 6.1 & 6.4 & 6.8 & 7.2 \\
Hardened air content (\%) & 7.2 & 7.1 & 7.4 & 7.9 \\
Specific surface (mm $^{-1}$ ) & 28.4 & 31.3 & 36.9 & 37.2 \\
Spacing factor (mm) & 0.301 & 0.246 & 0.193 & 0.177 \\
\hline
\end{tabular}

As seen from Table 3.5, the hardened air content results fluctuated between 7.1\% and 7.9\% based on MA type. There were slight increments in the values of hardened air contents of ECC mixtures over fresh air contents, and the increased values were still acceptable for F/T durability (ACI Committee 345, 2011). Considering the effects of different types of MAs on hardened air contents of ECC mixtures, similar behavior observed from the fresh air content results was valid, with ECC_S specimens showing higher values compared to other mixtures. ECCs with slag showed the highest hardened air content and the lowest spacing factor. In this regard, one might assume that for similar hardened air contents, lower spacing factor values of ECC_S specimens $(0.177 \mathrm{~mm})$ indicate closely spaced bubbles with reduced average sizes, which is favorable for good F/T durability. It is a well-known fact that for superior frost durability, degree of hydration and the microstructural state of concrete at the moment of freezing are decisive. Therefore, the hydration capabilities of different MAs with almost completely different chemical natures were expected to affect the overall air-void parameter to great extent. It should also be noted that the significantly high pozzolanic capacity of different fly ash types (especially Class-F fly ash) is likely to decrease hardened air content for much longer periods (more than 14 days) compared to slag particles, which have high cementitious value. Individual assessments of spacing factor and specific surface results of different mixtures show that while all mixtures failed to satisfy the recommended specific surface value of $25.0 \mathrm{~mm}^{-1}$ for adequate frost durability, only those with Class-F fly ash (ECC_L and ECC_M) failed the recommended spacing factor value of $0.2 \mathrm{~mm}$ (Powers 1964, 1965). Although some of the air-void parameters of certain ECC mixtures were beyond the generally accepted values due to lack of air entraining admixture, high pozzolanicity, and 
inadequate initial curing period, all ECC specimens exhibited satisfactory frost durability, which will be further detailed in forthcoming sections. Overall, these findings suggest that air-void parameters are not the sole decisive factors in frost durability of ECCs.

\subsubsection{Water sorptivity}

3.2.4.1. Water sorptivity of microcracked cementitious composites before frost deterioration

Table 3.6 shows crack characteristics (number of cracks and average crack widths) and sorptivity indexes of sound and pre-loaded ECC mixtures before frost deterioration. Water sorptivity results of sound ECC mixtures before exposure to F/T cycles for ECC_L, ECC_M, ECC_H and ECC_S specimens were $0.0551,0.0677,0.0375$ and $0.0056 \mathrm{~mm} / \mathrm{min}^{1 / 2}$, respectively. Although ECC_H specimens showed lower sorptivity results than ECC mixtures with Class-F fly ash, ECC_S specimens showed significantly lower results (more than 10 times lower than ECC_L and ECC_M) than the rest of the mixtures. This finding was expected; it was presumed that finer and highly reactive slag particles would significantly modify matrix properties, especially at the early stages of hydration, in favor of attaining disconnected, reduced numbers of capillary pores. The Class-C type fly ash particles appeared to be effective in reducing the sorptivity results to a certain level, thanks to the simultaneous effects of pozzolanic capacity and cementing capability, while highly pozzolanic Class-F fly ash particles required more initial curing for equivalent results.

The effect of pre-loading on sorptivity results of ECC specimens without frost deterioration was another point requiring further attention in terms of the results presented in Table 3.6. The table shows that no matter what type of MA was used, considerable increments in sorptivity results occurred with applied pre-loading. For example, while the average sorptivity result of sound ECC_S specimens was $0.0056 \mathrm{~mm} / \mathrm{min}^{1 / 2}$, the same value reached $0.0065 \mathrm{~mm} / \mathrm{min}^{1 / 2}$ based on the crack characteristics. Moreover, increased crack numbers were accompanied by exponential increments in sorptivity results of all ECC mixtures. For a better simulation of this behavior, sorptivity results were plotted against number of cracks (Figure 3.5). This figure shows an exponential relationship between both parameters with the least correlation factor $\left(\mathrm{R}^{2}\right)$ of 0.92 .

This finding corresponds well with a study investigating the influence of microcracking on sorptivity results of Class-F fly ash bearing ECCs with a $0.27 \mathrm{~W} / \mathrm{CM}$ ratio and a $1.2 \mathrm{FA} / \mathrm{PC}$ ratio of (ECC-1 [similar to ECC_L and ECC_M in this study]) (Sahmaran and Li 2009). 
Table 3.6: Crack characteristics and sorptivity indexes of ECC specimens

\begin{tabular}{|c|c|c|c|c|c|c|c|c|c|c|}
\hline \multirow{3}{*}{ Mix ID. } & \multirow{3}{*}{$\begin{array}{c}\text { \# of } \\
\text { specimen }\end{array}$} & \multirow{3}{*}{ \# of cracks } & \multirow{3}{*}{$\begin{array}{c}\text { Average } \\
\text { crack } \\
\text { width } \\
\text { ( } \mu \mathrm{m})\end{array}$} & \multicolumn{7}{|c|}{ Water sorptivity $\left(\mathrm{mm} / \mathrm{min}^{1 / 2}\right)$} \\
\hline & & & & \multicolumn{7}{|c|}{ \# of freeze-thaw cycles } \\
\hline & & & & $\mathbf{0}$ & 50 & 100 & 150 & 200 & 250 & 300 \\
\hline \multirow{6}{*}{ ECC_L } & 2 & 0 & 0 & 0.0551 & 0.0200 & 0.0173 & 0.0181 & 0.0214 & 0.0261 & 0.0378 \\
\hline & 1 & 2 & 70 & 0.0641 & 0.0252 & 0.0219 & 0.0264 & 0.0288 & 0.0386 & 0.0496 \\
\hline & 1 & 4 & 60 & 0.0762 & 0.0224 & 0.0212 & 0.0215 & 0.0371 & 0.0433 & 0.0642 \\
\hline & 1 & 6 & 63 & 0.1084 & 0.0429 & 0.0522 & 0.0591 & 0.0614 & 0.0718 & 0.0799 \\
\hline & 1 & 8 & 65 & 0.1552 & 0.0614 & 0.0574 & 0.0533 & 0.0677 & 0.0832 & 0.0979 \\
\hline & 1 & 12 & 67 & 0.2340 & 0.0884 & 0.0662 & 0.0715 & 0.0825 & 0.0940 & 0.1095 \\
\hline \multirow{6}{*}{ ECC_M } & 2 & 0 & 0 & 0.0677 & 0.0288 & 0.0308 & 0.0346 & 0.0470 & 0.0481 & 0.0569 \\
\hline & 1 & 4 & 63 & 0.0793 & 0.0338 & 0.0441 & 0.0556 & 0.0651 & 0.0748 & 0.0828 \\
\hline & 1 & 5 & 77 & 0.1150 & 0.0579 & 0.0648 & 0.0695 & 0.0801 & 0.0905 & 0.1068 \\
\hline & 1 & 6 & 61 & 0.1397 & 0.0513 & 0.0568 & 0.0623 & 0.0826 & 0.0926 & 0.1135 \\
\hline & 1 & 8 & 75 & 0.1800 & 0.0883 & 0.0614 & 0.0692 & 0.0782 & 0.0957 & 0.1217 \\
\hline & 1 & 11 & 73 & 0.2270 & 0.0973 & 0.1043 & 0.1173 & 0.1244 & 0.1401 & 0.1418 \\
\hline \multirow{6}{*}{ ECC_H } & 2 & 0 & 0 & 0.0375 & 0.0183 & 0.0171 & 0.0161 & 0.0139 & 0.0213 & 0.0274 \\
\hline & 1 & 1 & 55 & 0.0500 & 0.0490 & 0.0476 & 0.0305 & 0.0444 & 0.0542 & 0.0709 \\
\hline & 1 & 2 & 80 & 0.0642 & 0.0423 & 0.0481 & 0.0431 & 0.0482 & 0.0641 & 0.0758 \\
\hline & 1 & 4 & 64 & 0.0887 & 0.0465 & 0.0490 & 0.0470 & 0.0732 & 0.0942 & 0.1174 \\
\hline & 1 & 5 & 68 & 0.1017 & 0.0489 & 0.0438 & 0.0569 & 0.0713 & 0.0968 & 0.1168 \\
\hline & 1 & 7 & 67 & 0.1421 & 0.0782 & 0.0731 & 0.0715 & 0.0922 & 0.1033 & 0.1296 \\
\hline \multirow{6}{*}{ ECC_S } & 2 & 0 & 0 & 0.0056 & 0.0065 & 0.0142 & 0.0190 & 0.0251 & 0.0242 & 0.0377 \\
\hline & 1 & 1 & 64 & 0.0095 & 0.0152 & 0.0159 & 0.0235 & 0.0344 & 0.0395 & 0.0458 \\
\hline & 1 & 2 & 68 & 0.0145 & 0.0242 & 0.0280 & 0.0320 & 0.0472 & 0.0405 & 0.0674 \\
\hline & 1 & 4 & 80 & 0.0215 & 0.0263 & 0.0297 & 0.0311 & 0.0465 & 0.0596 & 0.0732 \\
\hline & 1 & 5 & 71 & 0.0395 & 0.0500 & 0.0539 & 0.0615 & 0.0720 & 0.0792 & 0.0864 \\
\hline & 1 & 7 & 65 & 0.0615 & 0.0763 & 0.0782 & 0.0975 & 0.1050 & 0.1235 & 0.1490 \\
\hline
\end{tabular}

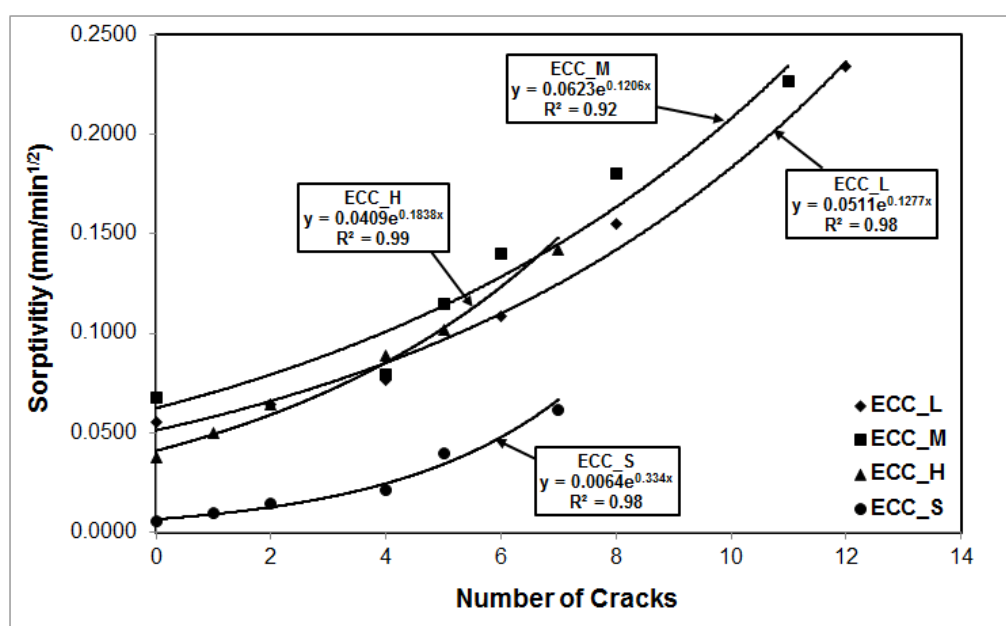

Figure 3.5: Changes in sorptivity results with the number of cracks

Despite the closely corresponding exponential relationship between increased crack numbers and sorptivity results, changes in increment rate varied depending on the type of MA. This finding is further detailed in Figure 3.6, where percentage changes in sorptivity results are plotted with 
respect to different crack numbers. In accordance with this figure, one might easily conclude that increments of sorptivity results for slag incorporating ECCs were much more significantly affected by crack formation. For example, when specimens with a minimum number of cracks ( 1 to 4 ) were evaluated, percentage increments in sorptivity results of sound specimens were $16 \%, 17 \%, 33 \%$ and 70\% for ECC_L, ECC_M, ECC_H and ECC_S mixtures, respectively. Increasing the number of cracks over the specimens did not change the observed behavior of ECC_S specimens, although the percentage increments were much more evident. For example, in specimens with the maximum number of cracks (7 to 12), sorptivity results for ECC_L, ECC_M, ECC_H and ECC_S specimens escalated by 325\%, 235\%, 279\%, 998\% in accordance with the sound specimens, respectively.

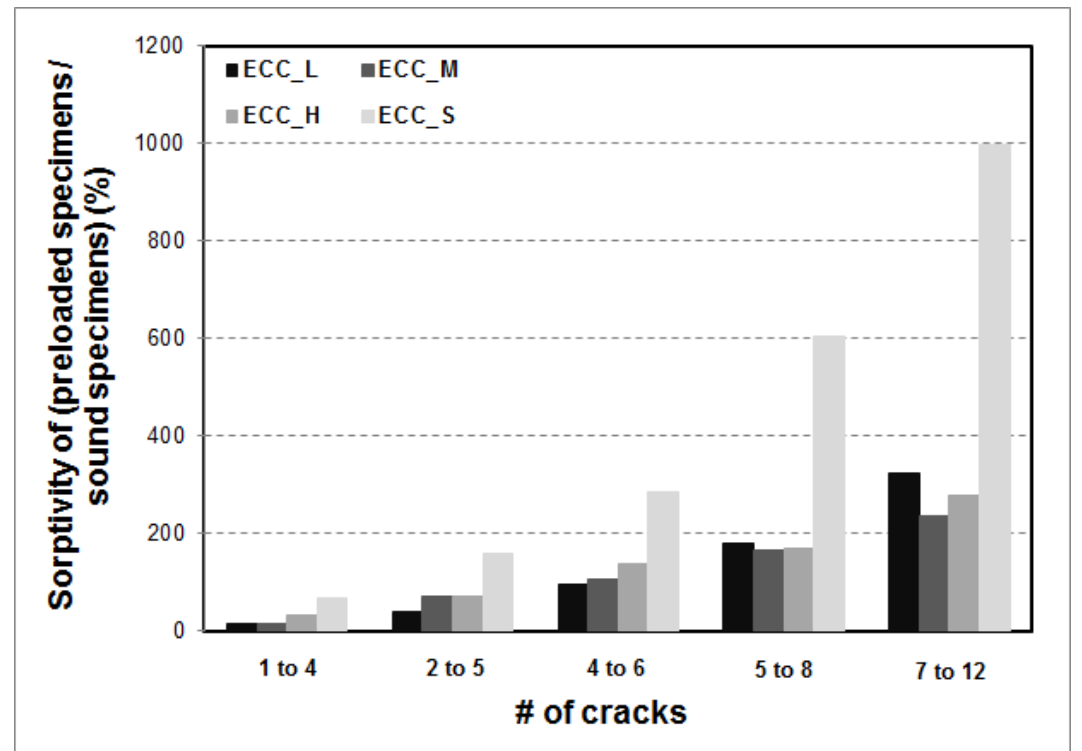

Figure 3.6: Percent changes in water sorptivity results of ECCs with different crack numbers before frost deterioration

Although sorptivity behavior of different mixtures upon exposure to mechanical pre-loading is very clear, it must be noted that for an exact evaluation of sorptivity characteristics, it is not entirely accurate to comment on the differences in percentage changes, since these results are markedly influenced by the changes in crack characteristics. To account for this drawback during the comparison of results, specimens from different mixtures with the same crack numbers must be compared. For Table 3.4, this comparison can be made by focusing on ECCs with four cracks. As seen from the table, for a given number of cracks, the average crack widths of ECC_S specimens (80 $\mu \mathrm{m})$ were wider than for those with different fly ash types (60, 63 and $64 \mu \mathrm{m}$ for ECC_L, ECC_M and ECC_S, respectively), which can be attributed to the increased chemical bonding 
between fiber and matrix with the use of slag, resulting in lower tensile ductility and more localized cracks. Overall, these results are interesting since increased crack widths are likely to reduce the rate of water suction into cracks. A possible explanation for microcracked ECC_S specimens exhibiting higher increments in sorptivity results could be related to the length and tortuosity of cracks. As seen from Figure 3.7, water movement follows a much more tortuous path into microcracked ECC_S prisms, spreading into nearly the whole cross-section, unlike ECC_L prisms.

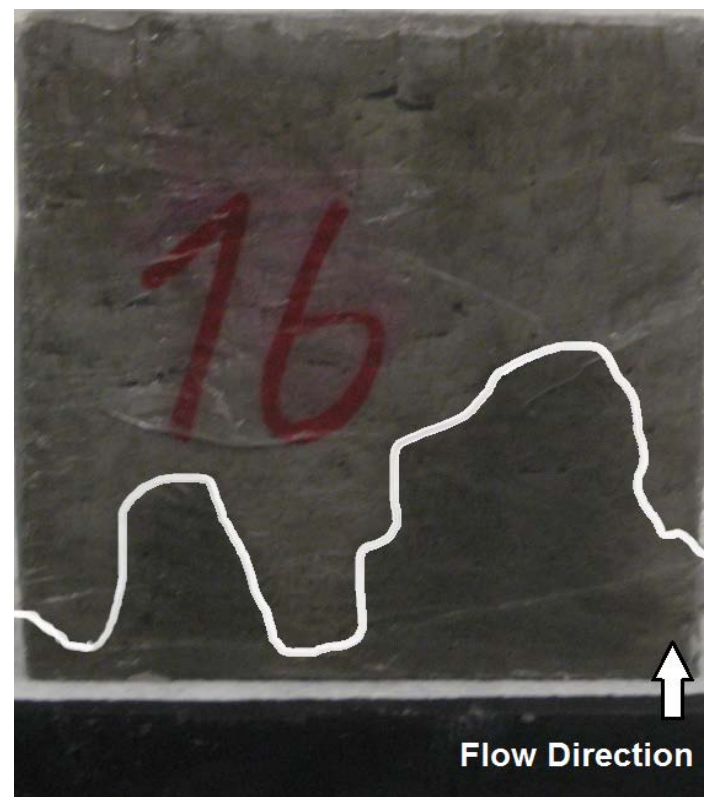

ECC_L

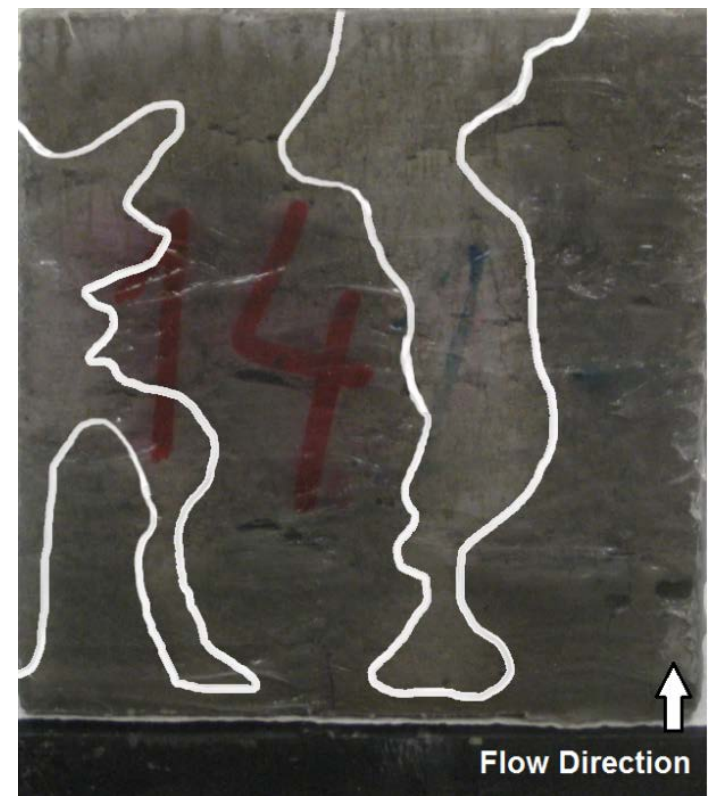

ECC_S

Figure 3.7: ECC_L and ECC_S prisms $(75 \times 75 \times 50 \mathrm{~mm})$ after 6 hours of water exposure

However, it must be noted that due to the relatively low sorptivity results of sound ECC_S specimens, even the lowest increment in values caused dramatic escalations in percentage calculations, despite the fact that the lowest sorptivity values for all crack numbers were from the same mixture. It can therefore be concluded that along with the intentionally imposed microcracks, interconnectivity of capillary pores (connected with the further binding capability of different MAs) is a governing parameter in the realization of actual water sorptivity results.

\subsubsection{Water sorptivity of microcracked cementitious composites after frost deterioration}

Sorptivity measurements of ECC mixtures were recorded after each $50 \mathrm{~F} / \mathrm{T}$ cycles until the completion of $300 \mathrm{~F} / \mathrm{T}$ cycles; results are presented in Table 3.6. To better simulate the results, sorptivity data were plotted against different numbers of F/T cycles in Figure 3.8. 

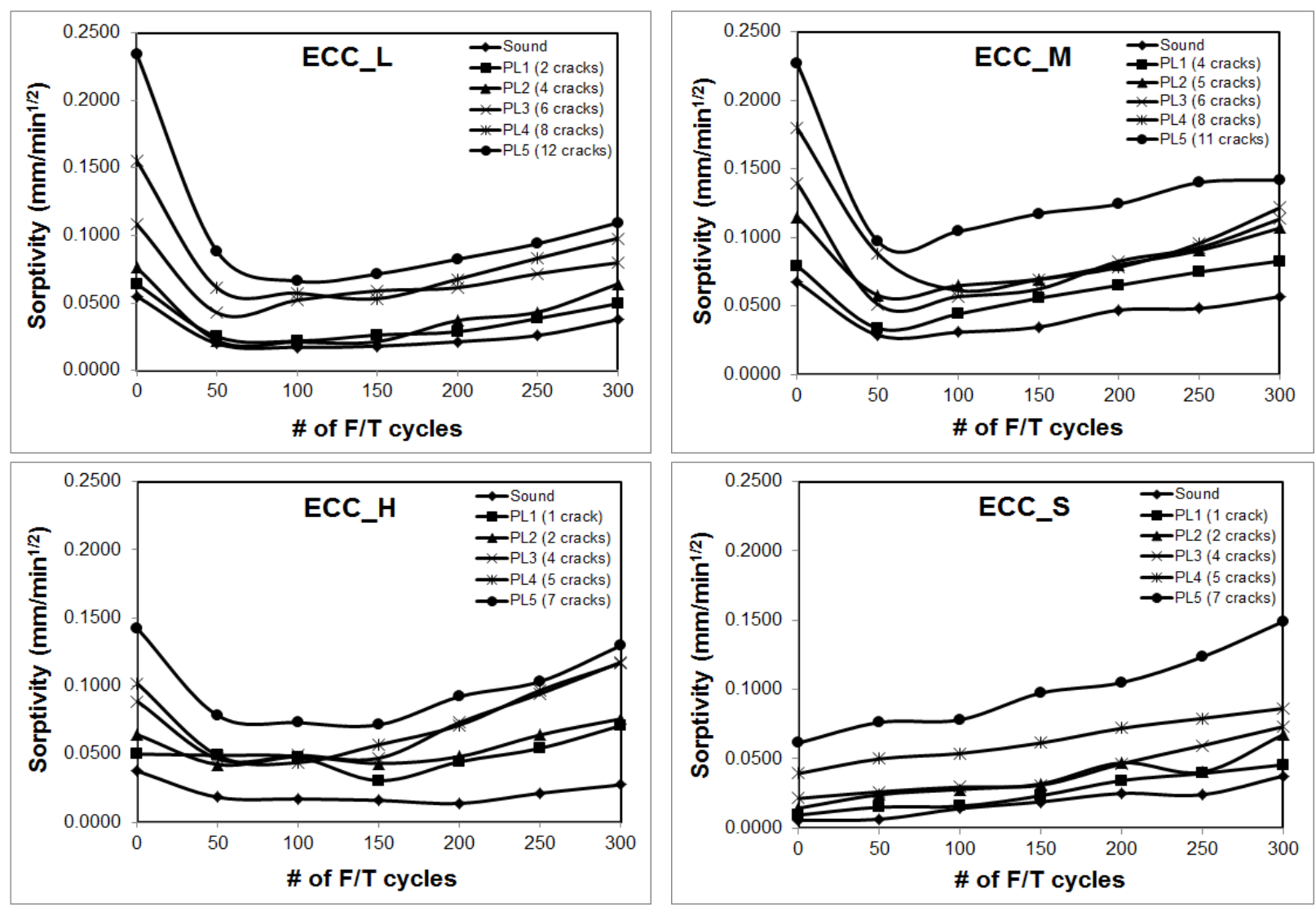

Figure 3.8: Water sorptivity measurements after the application of F/T cycles

Evaluation of the sound specimens showed that after the first $50 \mathrm{~F} / \mathrm{T}$ cycles, sorptivity results decreased sharply for specimens with different fly ash types, although this reduction was slightly less pronounced for ECC_H specimens. For example, the reduced sorptivity results after 50 F/T cycles were $0.0200,0.0288$ and $0.0183 \mathrm{~mm} / \mathrm{min}^{1 / 2}$ for ECC_L, ECC_M and ECC_H specimens, which means that original values decreased by $64 \%, 58 \%$ and $51 \%$ for the given mixtures, respectively. These findings show that during the $50 \mathrm{~F} / \mathrm{T}$ cycles (nearly 10 days), specimens reached a state where improvements in sorptivity measurements caused by further hydration were more effective than the deterioration due to frost action. It appears that the highly pozzolanic ingredients (Class-F fly ash) of the ECC_L and ECC_M mixtures made a more notable impact on the sorptivity results than the ECC_H mixture with high concentrations of less pozzolanic but more cementitious Class-C fly ash particles. In addition, for some of the mixtures, further frost action led to a further decrease in sorptivity results of sound specimens after the first $50 \mathrm{~F} / \mathrm{T}$ cycles. For example, after an additional $50 \mathrm{~F} / \mathrm{T}$ cycles (for a total of $100 \mathrm{~F} / \mathrm{T}$ cycles) sorptivity results of ECC_L sound specimens went down to $0.0173 \mathrm{~mm} / \mathrm{min}^{1 / 2}$, while for ECC_H sound specimens, a 
minimum of $0.0139 \mathrm{~mm} / \mathrm{min}^{1 / 2}$ was reached after an additional $150 \mathrm{~F} / \mathrm{T}$ cycles (a total of $200 \mathrm{~F} / \mathrm{T}$ cycles). These findings show that in order to prevent severe frost deterioration in sound ECC specimens, the further binding capability of MA and its contribution to the matrix maturity were important for the sensitivity of sorptivity measurements. On the other hand, for ECC_S, the opposite behavior was observed in specimens with fly ash; sorptivity results of sound specimens showed slight increments (from 0.0056 to $0.0065 \mathrm{~mm} / \mathrm{min}^{1 / 2}$ ) with $50 \mathrm{~F} / \mathrm{T}$ cycles. As previously mentioned, sound ECC_S specimens incorporating highly reactive slag particles exhibited the lowest sorptivity results among all mixtures. However, unlike specimens with fly ash, the higher reactivity of slag in ECC_S specimens prevented further curing in the first 50 F/T cycles. It kept them from attaining better matrix properties and avoiding the negative effects of frost action, causing the final results to be slightly higher. Overall, even after $300 \mathrm{~F} / \mathrm{T}$ cycles, the values of all ECC mixtures excluding ECC_S were lower than those of sound specimens before exposure to frost action, which means that for certain mixtures, frost deterioration was counteracted by the enhancements achieved in the matrix properties during this period. Like the sound ECC specimens, pre-loaded ECCs produced with different fly ash types showed marked decrements in sorptivity results after certain periods of $\mathrm{F} / \mathrm{T}$ exposure (especially during the first $50 \mathrm{~F} / \mathrm{T}$ cycles), depending on the extent of damage introduced to the specimens. Although individual data related to sorptivity measurements of pre-loaded specimens are provided in Table 3.6, changes in the results with the applied F/T cycles are clearer in Figure 3.8. One key element in this figure shows that drops in sorptivity results during the first $50 \mathrm{~F} / \mathrm{T}$ cycles were considerably sharper for specimens with higher numbers of cracks. Although this behavior of highly cracked specimens with different fly ash types was not true in all cases, it should be mentioned that sorptivity measurements were not singly dependent on crack numbers. Other parameters such as crack width, length and tortuosity of the crack path were just as important and were likely responsible for minor deviations from the abovementioned statement. Furthermore, sorptivity results of pre-loaded specimens were reduced further beyond the first 50 F/T cycles. Based on the crack characteristics, some of the ECC_L, ECC_M and ECC_H mixtures exhibited further enhancements in sorptivity results to the end of 100 and 150 F/T cycles, beyond which the values started to increase again. Enhanced sorptivity results (sometimes to the end of $150 \mathrm{~F} / \mathrm{T}$ cycles) in microcracked ECCs were surprising, especially when specimens were subjected further cyclic F/T conditioning. One possible explanation for the observed behavior is the self-healing of microracks and the ongoing hydration of the cementitious 
matrix itself during the thawing period of cyclic testing. The beneficial effects of self-healing also contributed to higher crack numbers and higher rates of improvement in sorptivity results. This could be due to the fact that a higher number of microcracks provides easier access for the necessary compounds required for self-healing, and creates additional space for the new selfhealing products. Self-healing probability also increases statistically with the increased number of cracks. Unlike all other pre-loaded ECC specimens, the pre-loaded ECC_S specimens did not show reductions in sorptivity measurements under F/T cycles. Adversely, results worsened with higher crack numbers and prolonged F/T cycles. Although partial closure of cracks was expected in microcracked ECC_S specimens, as well as in specimens with different fly ash types under F/T effect (Sahmaran et al. 2013), the rate was not adequate to counteract the negative effects of frost action in terms of sorptivity results. This finding could be related to the formation of different final self-healing products from ECCs with fly ash and slag. According to Sahmaran et al. (2013), major self-healing products observed in ECCs with different fly ash types are mainly C-S-H gels; in ECCs with slag, the product is mainly calcite. However, for the formation of calcite, dissolution of $\mathrm{CO}_{2}$ at a certain humidity level is necessary before reacting with leached-away free $\mathrm{Ca}^{+}$ions, a process that is not possible in fully saturated conditions. Therefore, although microcracked ECC_S specimens had high self-healing capability, it appears that being under water during the entire F/T test kept self-healing (calcite formation in this case) performance to a minimum. Moreover, ECC_S specimens performed most of their hydration reactions by the end of 14 days, leading to relatively low sorptivity results which are unlikely to be further improved upon with prolonged F/T cycles. Thus, significantly reduced self-healing and further hydration performance of ECC_S specimens due to testing caused frost action to take over the final sorptivity results simply by increasing them after each F/T cycle. Nevertheless, it is notable that increased sorptivity results did not keep ECC_S specimens from having similar and/or lower sorptivity results than ECCs with different fly ash types (Table 3.6). According to other researchers (Tsivilis et al. 2003; Chindaprasirt et al. 2005; Mohammed et al. 2002), concrete with a water to cement ratio of 0.400.50 shows a sorptivity index of about $0.2300 \mathrm{~mm} / \mathrm{min}^{1 / 2}$, which is almost two times the highest value $\left(0.1490 \mathrm{~mm} / \mathrm{min}^{1 / 2}\right.$ from ECC_S specimen with seven cracks after $300 \mathrm{~F} / \mathrm{T}$ cycles) obtained from the ECCs produced for this study. Therefore, these results clearly indicate that no matter what the type of MA used during proportioning, ECCs satisfied marked frost durability in terms of 
sorptivity results compared to normal sound concrete, even after 300 severe F/T conditioning cycles.

\subsection{Chapter summary}

In this chapter, effects of microcracking on chloride ion penetrability of ECC specimens incorporating different SCMs were studied along with some of the basic mechanical properties of specimens. Also, this Chapter focused on the frost durability of cementitious composites produced with mineral admixtures (MAs) with significantly different chemical compositions. Although ASTM C 666 suggests resonant frequency and dynamic modulus of elasticity measurements for the assessment of freeze-thaw durability, this study used water sorptivity measurements since damage caused by repetitive freezing and thawing started from the exposed surface and spread further under the influence of capillary action. Fresh and hardened air-void characteristics of ECCs with different MAs were compared. 


\section{Chapter 4}

\section{Effect of Self-Healing on the Different Transport Properties of Cementitious Composites incorporating different mineral admixtures}

\subsection{Introduction}

This Chapter focuses on the effects of self-healing on the different transport properties of microcracked Engineered Cementitious Composites (ECCs) with different maturity levels and incorporating three different mineral admixtures (One type of ground granulated blast furnace slag (S) along with two different fly ashes (FA)) with greatly varying chemical compositions. The effect of self-healing capability on transport properties was assessed using water sorptivity and rapid chloride permeability tests (RCPT).

\subsection{Experimental program}

\subsubsection{Materials, mixture proportions and basic mechanical properties}

Ordinary Portland cement (PC), silica sand with maximum aggregate size (MAS) of $400 \mu \mathrm{m}$ and water absorption capacity of $0.3 \%$, water, poly-vinyl alcohol (PVA) fibers with a diameter of 39 $\mu \mathrm{m}$, nominal tensile strength of $1610 \mathrm{MPa}$ and specific gravity of 1.3 , and high range water reducing admixture (HRWRA) were used for the production of the ECC mixtures. In addition, different mineral admixtures (MA) representing greatly varying chemical compositions were incorporated into the mixtures. One type of ground granulated blast furnace slag (S, [ECC_S]) along with two different fly ashes (FA) were selected as different MAs. The fly ashes were low lime fly ash ([FA-L], ECC_L) and high lime fly ash ([FA-H], ECC_L). The chemical and physical properties of the Portland cement and mineral admixtures are presented in Table 3.1, along with the particle size distributions of solid ingredients in Figure 3.1. The ECC mixtures were produced with a water to cementitious materials (PC+MA) ratio (W/CM) of 0.27 and a mineral admixture (i.e. FA or S) to Portland cement ratio (MA/PC) of 1.2, by mass. Mixture proportion details are shown in Table 3.2. 


\subsubsection{Sample preparation, pre-cracking and methods for self-healing evaluation}

As previously mentioned, this chapter concentrates on the sorptivity measurements of sound and pre-cracked ECC specimens to evaluate self-healing performance. Rapid chloride permeability tests (RCPTs) and the assessment of crack characteristics were also performed. $360 \times 75 \times 50 \mathrm{~mm}$ prism (length $\times$ depth $\times$ width) specimens with different ECC mixtures were produced for sorptivity measurement. After 24 hours inside the molds at $23 \pm 2{ }^{\circ} \mathrm{C}$ and $50 \pm 5 \% \mathrm{RH}$, specimens were removed from the molds and placed in impermeable plastic bags at $23 \pm 2{ }^{\circ} \mathrm{C}$ and $95 \pm 5 \% \mathrm{RH}$ cured for 7, 28 and 90 days. Before the tests, three separate specimens from each mixture and testing age were loaded up to failure under four-point bending loading for the determination of ultimate bending deformation capacities. The results were in the range of 1.7 to $4.8 \mathrm{~mm}$, depending on the usage of different mineral admixtures and initial curing age. Therefore, $80 \%$ of the ultimate bending deformation capacity of ECCs was set as a pre-cracking limit for all specimens. After each maturity level (7, 28 and 90 days) was reached, specimens were pre-loaded up to $80 \%$ of their four-point bending deformation capacity to obtain cracks with different characteristics. After the introduction of pre-loading, prisms measuring $75 \times 75 \times 50 \mathrm{~mm}$ were cut from the central portions of each specimen to evaluate sorptivity properties. Sorptivity tests were performed using four different prisms for each age, following the procedures of ASTM C 1585. For comparison, two out of the four prisms were kept sound (uncracked) and the rest were pre-loaded as described above. Special attention was paid to the introduction of cracks with varying numbers and widths during pre-loading. For the evaluation of self-healing through sorptivity measurements, 7, 28 and 90-day old sound and pre-loaded specimens were individually subjected to continuous water (CW) curing at $23 \pm 2{ }^{\circ} \mathrm{C}$ for an additional 7, 30, 60 and 90 days, and the tests were repeated after the completion of additional curing periods. Before the commencement of sorptivity testing, specimens were left to dry in an oven at $50 \pm 5^{\circ} \mathrm{C}$ until a constant weight was obtained. After all specimens were completely dry, they were immersed in water by 3-5 mm, as seen in Figure 3.2. To ensure one-directional flow through the specimens and reduce the chance of water evaporation, prisms were laterally sealed with silicon coating. Changes in mass of the ECC prism specimens that were left to absorb water through capillary action were recorded at time intervals of 1, 2, 3, 4, 6, 8, 12, 16, 20, 25, 36, 49, 64, 81, 120 and 360 minutes. Additionally, changes in the widths of cracks formed over the specimens used for sorptivity tests were observed with a video microscope at the end of each pre-determined testing age. 
As another way of detecting self-healing performance, rapid chloride permeability tests (RCPTs) were performed in accordance with ASTM C 1202. After one day of curing in laboratory conditions at $23 \pm 2{ }^{\circ} \mathrm{C}$ and $50 \pm 5 \% \mathrm{RH}, \varnothing 100 \times 200 \mathrm{~mm}$ cylindrical specimens were removed from the molds and then kept in plastic bags at $23 \pm 2{ }^{\circ} \mathrm{C}$ and $95 \pm 5 \% \mathrm{RH}$ until the end of 7, 28 and 90 days. After the initial aging, $\varnothing 100 \times 200 \mathrm{~mm}$ cylinders were cut into $\varnothing 100 \times 50 \mathrm{~mm}$ pieces using a diamond blade saw. Before the pre-loading of specimens, the same procedures as those used for the prisms were followed to define the ultimate splitting tensile deformation capacities of different mixtures under splitting tensile loading. Results varied between $1.3 \mathrm{~mm}$ and $1.9 \mathrm{~mm}$, depending on different MAs and initial curing periods. Then, in order to create microcracks, $\varnothing 100 \times 50 \mathrm{~mm}$ ECCs were pre-loaded up to $80 \%$ of their splitting tensile deformation capacities at the ages of 7 , 28 and 90 days under splitting tensile loading at a loading rate of $0.005 \mathrm{~mm} / \mathrm{s}$. Six cylinders from each mixture, three of which were sound and three pre-loaded, were used for the RCPTs. Just as in the sorptivity measurements, tests were repeated after 7, 30, 60 and 90 days of CW exposure beyond initial curing periods to observe self-healing performance of ECCs. Although different crack characteristics were acquired with the application of pre-loading to ECCs with different mineral admixtures (MAs), the effect of self-healing on crack recoveries has not been accounted for in this study since this point has already been investigated in detail by other researchers (Sahmaran et al., 2013; Sahmaran et al., 2014).

\subsection{Results and discussion}

\subsubsection{Sorptivity test}

\subsubsection{Unhealed specimens}

Table 4.1 shows the crack characteristics and sorptivity index values of 7-, 28- and 90-day-old ECC specimens that were not subjected to further CW curing for self-healing assessment. As seen from the table, the type of mineral admixture and initial curing age (maturity level) are influential on sorptivity results. Considering the effects of initial curing on the results of sound specimens, it can clearly be stated that there were remarkable decrements in the values when curing ages were prolonged from seven to 90 days. For example, the average seven-day sorptivity index of the ECC_L specimens was $0.0787 \mathrm{~mm} / \mathrm{min}^{1 / 2}$, while the same value decreased by $61 \%$ and $87 \%$ to 0.0317 and $0.0099 \mathrm{~mm} / \mathrm{min}^{1 / 2}$ levels for the 28 and 90-day-old specimens, respectively. Although the differences in results varied greatly depending on the mineral admixture type, the general trend 
was irrespective of MA type and valid for all of the mixtures. The observed behavior of sound ECC specimens upon exposure to longer curing periods was an anticipated outcome due to continuing hydration reactions and pozzolanic capacity, both of which are the main mechanisms contributing to the attainment of dense microstructure and disconnected pores.

Table 4.1: Sorptivity indexes and crack characteristics and of ECC specimens

\begin{tabular}{|c|c|c|c|c|c|c|c|c|c|}
\hline $\begin{array}{l}\text { Initial } \\
\text { Curing }\end{array}$ & Mix ID. & $\begin{array}{c}\text { \# of } \\
\text { Cracks }\end{array}$ & $\begin{array}{c}\text { Average } \\
\text { Crack Width }\end{array}$ & $\begin{array}{c}\text { \# of } \\
\text { Specimens }\end{array}$ & \multicolumn{5}{|c|}{ Sorptivity $\left(\mathrm{mm} / \mathrm{min}^{1 / 2}\right)$} \\
\hline \multirow{10}{*}{7 Days } & \multirow{4}{*}{ ECC_L } & & & & 7 & $7+7$ & $7+30$ & $7+60$ & $7+90$ \\
\hline & & 0 & - & 2 & 0.0787 & 0.0400 & 0.0275 & 0.0113 & 0.0089 \\
\hline & & 2 & 75 & 1 & 0.0927 & 0.0465 & 0.0297 & 0.0187 & 0.0103 \\
\hline & & 6 & 65 & 1 & 0.1482 & 0.0610 & 0.0361 & 0.0201 & 0.0122 \\
\hline & \multirow{3}{*}{ ECC_H } & 0 & - & 2 & 0.0311 & 0.0280 & 0.0220 & 0.0145 & 0.0105 \\
\hline & & 3 & 50 & 1 & 0.0550 & 0.0390 & 0.0301 & 0.0271 & 0.0199 \\
\hline & & 6 & 75 & 1 & 0.0749 & 0.0590 & 0.0472 & 0.0398 & 0.0305 \\
\hline & \multirow{3}{*}{ ECC_S } & 0 & - & 2 & 0.0115 & 0.0061 & 0.0044 & 0.0040 & 0.0037 \\
\hline & & 2 & 100 & 1 & 0.0241 & 0.0097 & 0.0076 & 0.0063 & 0.0054 \\
\hline & & 6 & 75 & 1 & 0.0593 & 0.0192 & 0.0122 & 0.0097 & 0.0088 \\
\hline \multirow{10}{*}{28 Days } & \multirow{4}{*}{ ECC_L } & & & & 28 & $28+7$ & $28+30$ & $28+60$ & $28+90$ \\
\hline & & 0 & - & 2 & 0.0317 & 0.0290 & 0.0117 & 0.0097 & 0.0082 \\
\hline & & 2 & 65 & 1 & 0.0495 & 0.0320 & 0.0216 & 0.0129 & 0.0106 \\
\hline & & 6 & 65 & 1 & 0.0860 & 0.0490 & 0.0278 & 0.0167 & 0.0120 \\
\hline & \multirow{3}{*}{ ECC_H } & 0 & - & 2 & 0.0249 & 0.0235 & 0.0154 & 0.0129 & 0.0097 \\
\hline & & 3 & 80 & 1 & 0.0328 & 0.0311 & 0.0304 & 0.0230 & 0.0173 \\
\hline & & 6 & 60 & 1 & 0.0596 & 0.0403 & 0.0351 & 0.0291 & 0.0298 \\
\hline & \multirow{3}{*}{ ECC_S } & 0 & - & 2 & 0.0049 & 0.0043 & 0.0041 & 0.0039 & 0.0037 \\
\hline & & 2 & 85 & 1 & 0.0082 & 0.0074 & 0.0070 & 0.0065 & 0.0051 \\
\hline & & 6 & 65 & 1 & 0.0376 & 0.0354 & 0.0351 & 0.0349 & 0.0261 \\
\hline \multirow{10}{*}{90 Days } & \multirow{4}{*}{ ECC_L } & & & & 90 & $90+7$ & $90+30$ & $90+60$ & $90+90$ \\
\hline & & 0 & - & 2 & 0.0099 & 0.0088 & 0.0081 & 0.0082 & 0.0084 \\
\hline & & 2 & 80 & 1 & 0.0145 & 0.0118 & 0.0110 & 0.0102 & 0.0105 \\
\hline & & 5 & 60 & 1 & 0.0460 & 0.0389 & 0.0355 & 0.0282 & 0.0240 \\
\hline & \multirow{3}{*}{ ECC_H } & 0 & - & 2 & 0.0135 & 0.0121 & 0.0106 & 0.0103 & 0.0092 \\
\hline & & 2 & 70 & 1 & 0.0276 & 0.0231 & 0.0225 & 0.0211 & 0.0193 \\
\hline & & 5 & 55 & 1 & 0.0484 & 0.0412 & 0.0393 & 0.0329 & 0.0322 \\
\hline & \multirow{3}{*}{ ECC_S } & 0 & - & 2 & 0.0043 & 0.0038 & 0.0036 & 0.0030 & 0.0029 \\
\hline & & 3 & 65 & 1 & 0.0095 & 0.0086 & 0.0083 & 0.0077 & 0.0083 \\
\hline & & 8 & 85 & 1 & 0.0486 & 0.0435 & 0.0410 & 0.0361 & 0.0342 \\
\hline
\end{tabular}

Another notable point related to the sorptivity results of sound ECC mixtures is that ECC_S specimens at all maturity levels displayed the lowest sorptivity results, with values reaching $0.0115,0.0049$ and $0.0043 \mathrm{~mm} / \mathrm{min}^{1 / 2}$ levels at the end of 7, 28 and 90 days, respectively. It appears that slag particles, due to their significantly higher specific surface area (430 $\left.\mathrm{m}^{2} / \mathrm{kg}\right)$, contributed well to the advancements in hydration and pozzolanic reactions compared to fly ash. The higher 
fineness of slag particles is also of great importance due to so-called "filler effect," which can make major contributions to better distribution of particles in cementitious systems and denser microstructures. In the case of sorptivity results of ECC mixtures incorporating fly ash, there is a clear difference in the values at and after seven days of initial curing based on the type of fly ash. As seen from Table 4.1, the seven-day average sorptivity index of ECC_H specimens was less than $50 \%$ of that of the ECC_L, although the same finding was the opposite after 90 days of initial curing; the average sorptivity index of 90-day-old ECC_L specimens $\left(0.0099 \mathrm{~mm} / \mathrm{min}^{1 / 2}\right)$ was $27 \%$ less than that of ECC_H specimens $\left(0.0135 \mathrm{~mm} / \mathrm{min}^{1 / 2}\right)$. The lower sorptivity values of the ECC_H specimens after limited curing time is believed to be due to the dual effectiveness of high lime fly ash particles on both pozzolanicity and cementing behavior, which reduced the results, especially during early ages. However, the superior pozzolanic capacity of low lime fly ash appeared to take over the sorptivity results at later ages so that percentage changes between seven and 90 days were 87\% and 57\%, taking into account the ECC_L and ECC_H mixtures, respectively.

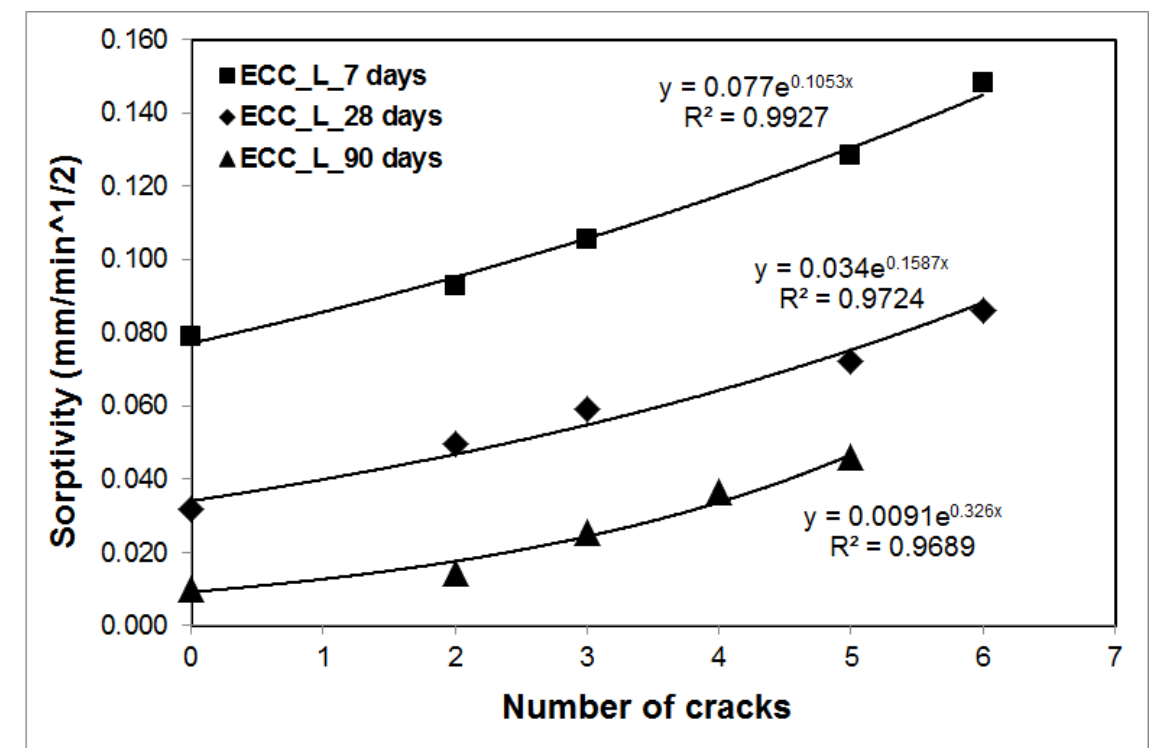

Figure 4.1: Typical example showing the changes in sorptivity indexes of ECC mixtures with the increase in number of cracks

Upon subjection to pre-loading, there were exponential increments in sorptivity results of ECC mixtures (Figure 4.1) without any regard to the type of MAs and initial curing period. This was an anticipated outcome since microcracks are likely to introduce additional pathways where water can be sucked into the material itself through capillary action. Increases in the number of cracks resulted in escalated sorptivity results as well. Taking the seven-day-old ECC_S mixtures as an 
example, the sorptivity results of specimens with two cracks were $0.0241 \mathrm{~mm} / \mathrm{min}^{1 / 2}$, although the same result was $0.0593 \mathrm{~mm} / \mathrm{min}^{1 / 2}$ in the case of specimens with six cracks. Moreover, this trend was valid for all of the mixtures and initial curing ages used in this study, showing that in highly damaged ECCs, capillary pores and microcracks tend to become saturated in a very limited time (Sahmaran and Li 2009).

When the combined influence of crack formation and initial curing periods on sorptivity values of ECCs is considered, it appears that cracking has a more profound effect on escalating the results of ECC specimens cured for a longer time. For example, when a comparison is made between seven- and 90-day-old ECC_L specimens with no cracks and the maximum number of cracks, sorptivity results of the 90-day-old pre-loaded specimens (365\% according to sound specimens) increased more than those of the seven-day-old specimens (88\% according to sound specimens) (Table 4.1). This modality held true for the rest of the mixtures, although crack characteristics altered significantly. The reason for the significant differences in the sorptivity results of sound and pre-loaded specimens cured for short and long periods could be related to the effect of different initial curing periods on the interconnection of capillary pores. Taking into account the specimens cured for shorter durations, there was less possibility of capillary pores being disconnected efficiently due to inadequate curing. Therefore, these disconnected pores were expected to have a stronger influence on water sorptivity, making the effect of microcracking on the results less visible for specimens of lower maturity. On the other hand, for specimens of higher maturity, disconnection of the capillary pores was more likely to occur, bringing mechanically-introduced microcracks into the more visible. However, it should be kept in mind that crack length and the tortuosity of the crack path are as important as crack width and could be responsible for the variations in overall sorptivity results.

Taking into consideration the effect of pre-loading on the sorptivity results of different mixtures, it is noticeable that pre-loading was much more efficient in the case of ECC_S specimens compared to ECC mixtures incorporating fly ash, especially at later ages. For example, while a $141 \%$ difference was found between the results of seven-day-old ECC_H specimens with no cracks (sound) and the specimens with the maximum number of cracks, the difference rose to 416\% for the ECC_S mixture. This is believed to be due to the effect of different mineral admixtures on the maturity of specimens and a possible explanation regarding this point has 
already been made in the previous paragraph. Despite the substantial increments in the sorptivity values of ECC_S specimens with applied pre-loading, it should not be overlooked that final results were lower than in the other mixtures after almost all ages and crack formations, which is related to the tightness of ECC_S matrices against water movement.

\subsubsection{Effects of self-healing}

Table 4.1 shows sorptivity measurements taken after the application of further 7, 30, 60 and 90 days of CW curing for the evaluation of self-healing in ECCs with the minimum number of cracks (2 to 3 ) and the maximum number of cracks (5 to 8), along with the sound ones (no cracks). For a better simulation of self-healing performance, percentage reductions in the results are presented in Figure 4.2. As can be concluded from both the table and figure, CW curing was quite influential on improving the sorptivity results, regardless of the type of mineral admixture and number of cracks. Generally speaking, there were sharp decreases in the sorptivity results of ECC specimens with the application of the first seven days of initial CW curing, although the dependency of improvements on different mineral admixture types and crack characteristics was quite clear. Faster improvements of results with the application of a limited duration of $\mathrm{CW}$ curing were most probably due to the larger availability of unhydrated particles ready to be further reacted in the systems. Moreover, the higher number of capillary pores belonging to younger specimens is more likely to be saturated faster and contribute to self-healing more efficiently.

The effect of different initial curing ages on the improvements in sorptivity results was profound. For example, the seven-day-old ECC_L specimens with the maximum number of cracks displayed a healing rate of $76 \%$ after 30 days of CW curing, while for the same specimens of 90-day age, healing after the same amount of time was limited to $23 \%$. This finding was also true for the rest of the ECCs, despite the fact that there were changes in crack numbers and widths. The finding was correlated with the gradual exhaustion of anhydrous materials in the composites cured for a longer time, which reduced the possibility for self-healing to occur. Additionally, this result was more pronounced in the sound specimens than in the load-induced ones (Figure 4.2), which could be due to the fact that water ingress through microcracks was easier and contributed well to carbonation, which is believed to be as powerful as hydration reaction (Edvardsen 1999). 

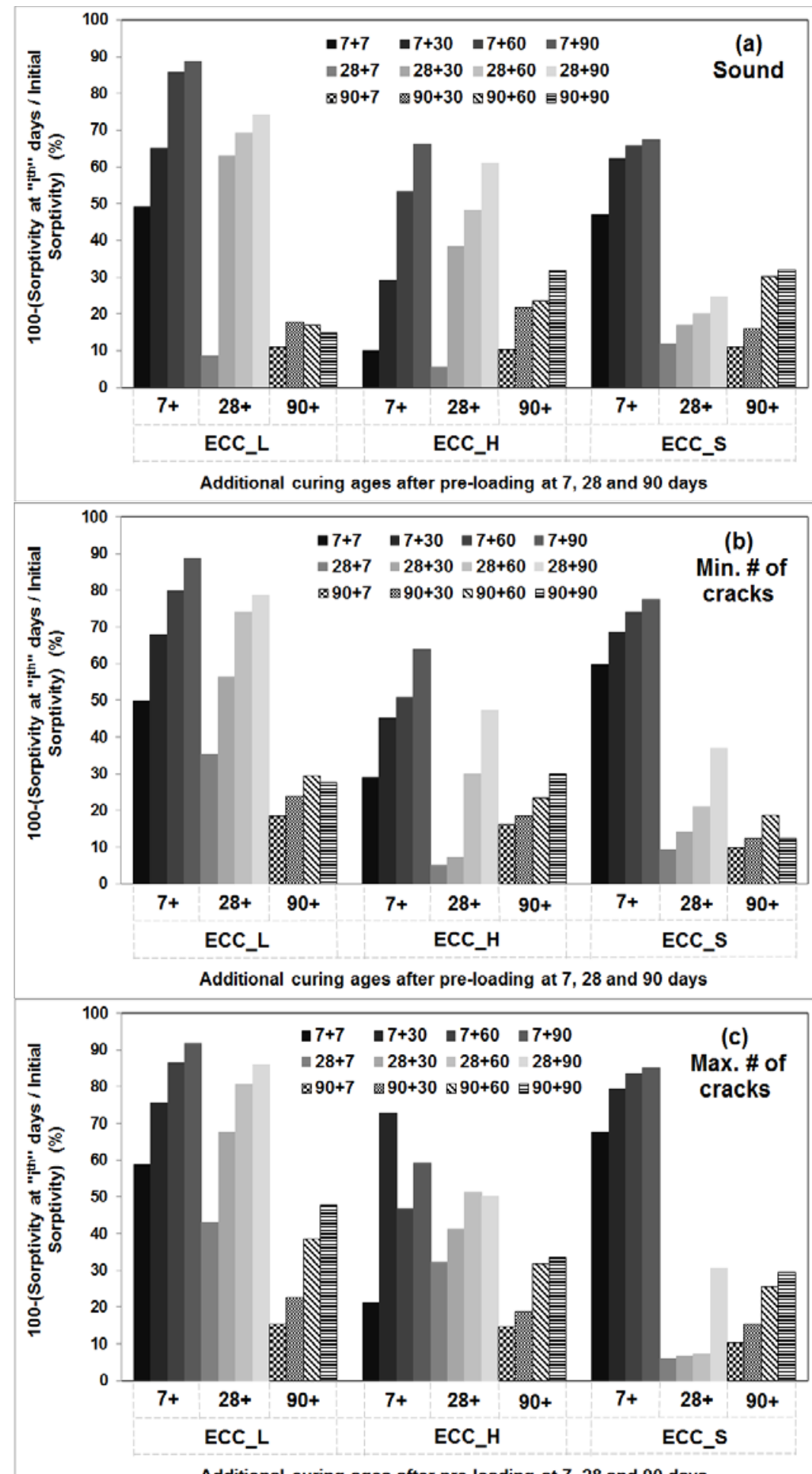

Additional curing ages after pre-loading at 7,28 and 90 days

Figure 4.2: Effect of self-healing on sorptivity results of ECCs with: a) no cracks b) min. \# of cracks c) max. \# of cracks

Accordingly, water movement into solid specimens is expected to be limited due to the significantly tight matrices of ECCs, which can restrict improvements in sorptivity results. Given 
the small dimensions of the tested specimens, however, it is likely that water moved more easily than expected and contributed to self-healing in solid specimens as well. Despite the reduced selfhealing rates with prolonged curing ages, it is important to note that self-healing at later ages was still at levels that cannot be overlooked, which provides invaluable information about the lifetime of the mechanism.

The influence of different mineral admixtures on the sorptivity results of additionally-cured specimens is another point requiring further discussion. As seen from Figure 4.2, the ECCs with low lime fly ash particles (ECC_L) showed superior self-healing performance, particularly when they were initially cured for a limited time, such as seven days. Considering the improvements in the results of the sound specimens, the performance of low lime fly ash particles was followed by that of high lime fly ash (ECC_H) and ground granulated blast furnace slag particles (ECC_S) in general. When the self-healing rates of pre-loaded specimens are taken into consideration, however, slag particles appear to surpass the performance of high lime fly ash and even low lime fly ash in some cases, when the initial curing period was limited. The self-healing performance of the ECC_S mixtures was surprising since it was presumed that specimens with slag would result in considerably lower amounts of anhydrous materials and higher matrix maturity along with higher total crack openings upon pre-loading. The enhanced self-healing performance of microcracked ECC_S specimens has been of issue in another study by Sahmaran et al. (2013). In that study, the authors concluded that since slag particles have lower amounts of $\mathrm{SiO}_{2}$ in their chemical compositions compared to other mineral admixtures, lower rates of pozzolanic reactions are anticipated, which would cause portlandite (as a product of cement hydration) to remain longer and in greater amounts in the cementitious matrices, leading to higher $\mathrm{pH}$ of the pore solution. When the $\mathrm{pH}$ value of pore solution is higher, carbonic acid (a product of the reaction between water and CO2) can disassociate faster in the forms of bicarbonate (HCO3-) and carbonate ions (CO32-), which are used for calcite formation by combining with leached-away $\mathrm{Ca}^{2+}$ ions from C$\mathrm{S}-\mathrm{H}$ gels and portlandite in the presence of $\mathrm{CO}_{2}$ abundant water (Jooss 2001; Edvardsen 1999). Therefore, the increased self-healing capability displayed by the ECC_S specimens was attributed to the calcite formation to a considerable extent. This behavior was also clear during visual observations, since calcite formation manifested itself as white residue over the microcracked specimens (Figure 4.4). 
(a) 7 days
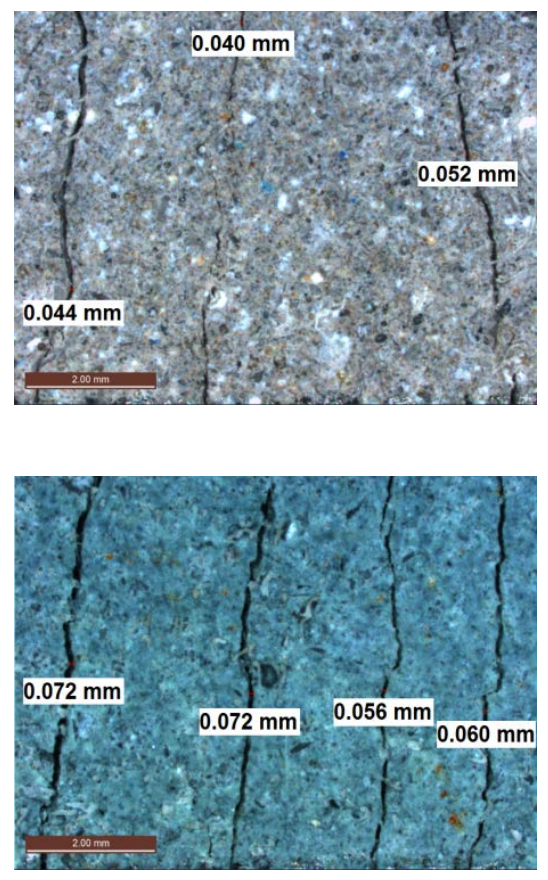

(b) $7+30$ days

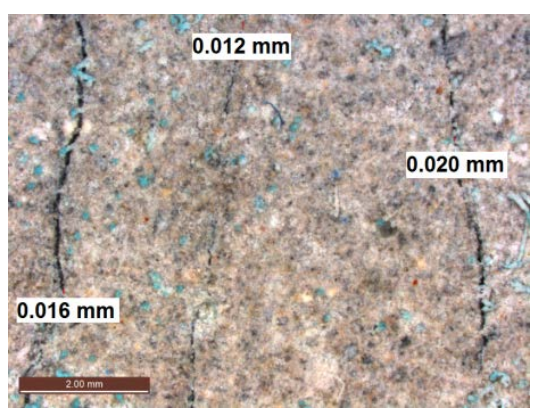

ECC_L

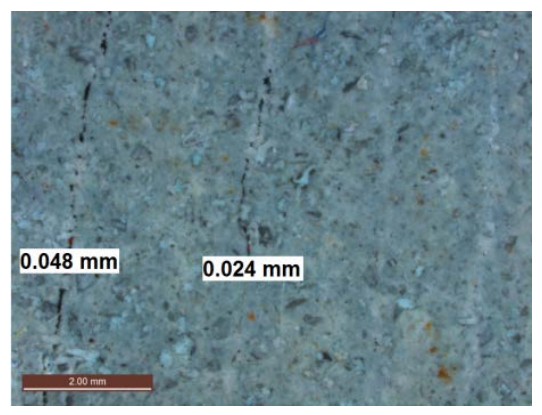

ECC_S (c) $7+90$ days
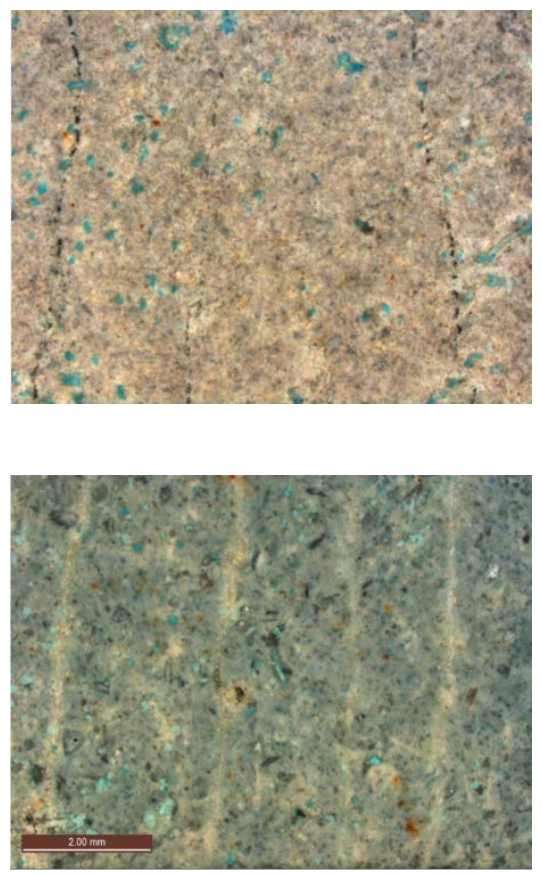

Figure 4.3: Typical self-healing of microcracks after exposure to CW curing

Generally speaking, self-healing in terms of the sorptivity results became more prominent when the specimens were subjected to pre-loading. In other words, as the microcracks over the specimens increased in number, the tendency for self-healing to take place got higher. However, this behavior was less pronounced in some of the specimens (ECC_H), due most probably to the varying crack characteristics (total crack width, crack length, tortuosity of crack paths etc.) and chemical composition of the mineral admixture, which are decisive on self-healing performance. A possible explanation for the enhanced self-healing performance monitored in the pre-loaded specimens could be that microcracks are more easily exposed to the ingress of $\mathrm{CO}_{2}$ dissolved water under CW curing, and they provide additional space for newly-formed self-healing products. However, it should not be forgotten that despite the higher rates of self-healing observed in the pre-loaded specimens, overall sorptivity indexes of highly-damaged specimens after the application of further CW curing was not as low as in the sound specimens. This is true for all of the specimens presented in this study. For example, average sorptivity results of seven-day-old sound ECC_L specimens decreased by $49 \%$, becoming $0.0400 \mathrm{~mm} / \mathrm{min}^{1 / 2}$ after only seven days of CW curing, while the same results of ECC_L specimens - which had the maximum number of cracks - decreased by $59 \%$, becoming $0.0610 \mathrm{~mm} / \mathrm{min}^{1 / 2}$ after the same further conditioning. 
Water movement into conventional concrete through capillary action has been studied extensively by other researchers. In one such study, Neville (1996) concluded that typical sorptivity of normal concrete with a water-to-cement $(\mathrm{W} / \mathrm{C})$ ratio of 0.40 is $0.0900 \mathrm{~mm} / \mathrm{min}^{1 / 2}$. According to other researchers (Tsivilis et al. 2003; Chindaprasirt et al. 2005; Mohammed et al. 2002), concrete with a W/C ratio of $0.40-0.50$ shows a sorptivity index of about $0.2300 \mathrm{~mm} / \mathrm{min}^{1 / 2}$. Based on these results from different studies, it can clearly be stated that even the highest sorptivity result $(0.0610$ $\mathrm{mm} / \mathrm{min}^{1 / 2}$ from seven-day-old ECC_L specimens with the maximum number of cracks) obtained after only seven days of CW curing is not particularly high, and it appears that sorptivity results (which are lower than the values of solid concrete mixtures) are attainable for almost-failed ECC specimens with a limited duration of environmental conditioning.

\subsubsection{Rapid chloride permeability test (RCPT)}

\subsubsection{Unhealed specimens}

The rapid chloride permeability test (RCPT) was used in addition to sorptivity measurements as another method to evaluate the self-healing capability of ECCs with different mineral admixtures. RCPT does not, in fact, measure the permeability of concrete. What it does measure is the resistance of concrete to electrical current. However, there is a close relationship between the resistivity of concrete and its permeability. The RCPT results of different ECC mixtures, which offer a good idea about specimens' resistivity against chloride ion ingress, are presented in Table 4.2. All of the values in this table were calculated by taking the average of three specimens. As seen, results differed significantly, considering the maturity levels and types of mineral admixtures used. For the RCPT results of seven-day-old sound specimens, results obtained were 7169, 4863 and 6397 C for the ECC_L, ECC_H and ECC_S mixtures, respectively. Although the ECC_S specimens were expected to display lower results, especially during the early stages of hydration, due to the enhanced cementing capability and high specific surface area of slag particles compared to different fly ashes, the results obtained from the ECC_H mixture were in opposition with this assumption. RCPT is an electrochemical test method influenced markedly by the changes in overall porosity of the concrete material and chemical composition of the pore solution. It may therefore be stated that in evaluating RCPT results, great attention must also be paid to the factors governing the chemical state of the pore solution (i.e. calcium silicate hydrate [C-S-H] gels, $\mathrm{CH}$ and alkalis). 
It is generally accepted that sodium and potassium ions present in cementitious matrices along with either sulfates or hydroxides contribute to the acceleration of early hydration, although they decelerate hydration at later ages (Jawed and Skalny 1978; Juenger and Jennings 2001). Moreover, it has been reported that alkalis are effective in modifying the morphology of calcium silicate hydrate (C-S-H) gels, leading to the formation of lath-like products, which have a higher degree of crystallinity (Mori et al. 1974; Richardson 2004). According to Bentz (2006), the formation of lath-like C-S-H gels in the presence of increased alkali amounts is the reason for the achievement of a depercolated (disconnected) capillary pore network. Despite the enhancements during the early stages of hydration, such microstructural alterations could be responsible for the changes in the transport properties of cementitious systems, which may explain the lower RCPT results obtained from ECC_H specimens with higher concentrations of alkali ions compared to ECC_S. Although it is believed to be less influential, the slightly lower specific gravity of FA-H particles compared to slag, which lead to the higher availability of these particles in the matrices per unit volume, could be another reason for the attainment of more compact systems and lower RCPT results of seven-day-old ECC_H specimens compared to ECC_S.

Table 4.2: Rapid chloride permeability test results of ECC specimens

\begin{tabular}{|c|c|c|c|c|c|c|c|}
\hline \multirow{2}{*}{ Mix ID. } & \multirow{2}{*}{$\begin{array}{l}\text { Initial } \\
\text { Curing Age }\end{array}$} & \multirow{2}{*}{ Virgin } & \multirow{2}{*}{ Pre-loaded } & \multicolumn{4}{|c|}{ Initial Curing + } \\
\hline & & & & 7 days & 30 days & 60 days & 90 days \\
\hline \multirow{3}{*}{ ECC_L } & 7 days & 7169 & 8318 & 5615 & 2475 & 907 & 511 \\
\hline & 28 days & 1615 & 3297 & 2442 & 887 & 577 & 429 \\
\hline & 90 days & 375 & 1308 & 1011 & 478 & 369 & 246 \\
\hline \multirow{3}{*}{ ECC_H } & 7 days & 4863 & 6289 & 3914 & 639 & 405 & 258 \\
\hline & 28 days & 956 & 1654 & 1021 & 517 & 296 & 234 \\
\hline & 90 days & 859 & 1511 & 1186 & 399 & 352 & 330 \\
\hline \multirow{3}{*}{ ECC_S } & 7 days & 6397 & 7888 & 3456 & 1599 & 1305 & 997 \\
\hline & 28 days & 2283 & 3549 & 2654 & 1780 & 1148 & 1007 \\
\hline & 90 days & 1195 & 3686 & 2297 & 1284 & 975 & 909 \\
\hline
\end{tabular}

As expected, prolonging CW curing resulted in significantly improved RCPT results due to the continuous evolution of the cementitious composites with time. Although the enhancements in RCPT results were close to each other, the results of mixtures incorporating low lime fly ash were generally lower at the end of 90 days. As seen from Table 4.2, beyond 90 days of initial curing until the end of 90 days of CW curing, the improvement in chloride ion penetrability of pre-loaded 
ECC_L specimens was 81\%, while the same values were $78 \%$ and $68 \%$ for ECC_H and ECC_S mixtures, respectively. Greater enhancements in RCPT results of mixtures incorporating FA-F particles were attributed to higher pozzolanic capacity and the ability of low calcium fly ash particles to remain for a longer period with no chemical interaction in the matrices. Despite the changes in improvements of RCPT results depending on different mineral admixture type, all of the 90-day-old results of sound specimens were in the range of very low chloride ion penetrability levels according to ASTM C 1202, except the ECC_S mixture, which fell in the low range.

With the application of pre-loading, chloride ion penetrability results increased to a certain level with no regard to initial curing period or mixture type. For instance, the seven-day average RCPT result of the ECC_L mixture increased from 7169 coulombs to 8318 coulombs when specimens were pre-loaded up to $80 \%$ of their splitting tensile deformation capacities. This behavior was much more pronounced when the specimens were aged further. When the ECC_S specimens are considered as an example, it is evident that pre-loading caused a $23 \%$ increase in chloride ion penetrability of seven-day-old specimens, although the increase in RCPT results escalated to 55\% and $140 \%$ of the initial values in the 28-day-old and 90-day-old specimens, respectively. This behavior could be attributed to the enhanced matrix maturity of the mixtures as a result of the extended curing time, which caused increased matrix fracture toughness values and triggered the formation of localized cracks with wider widths instead of steady-state multiple microcracking (Wang and Li 2007). It must also be stated that the higher percentage increments obtained with the applied pre-loading were caused due to the fact that calculations were made based on the relatively lower RCPT results obtained at later ages (28 and 90 days). For a better understanding of the application of pre-loading on RCPT results, the chloride ion penetrability levels of ECCs (according to ASTM C 1202) before and after cracking can be considered. For example, despite the 55\% increase in average RCPT results of the 28-day-old ECC_S specimens, values stayed in the moderate level before (2283 C) and after (3549 C) pre-loading. This behavior of 28- and 90day-old specimens was also valid for other mixture types, with slight variations, so that after preloading, all RCPT results stayed either in moderate $(<4000 \mathrm{C})$ or low $(<2000 \mathrm{C})$ chloride ion penetrability levels, regardless of mixture type. These findings prove that unlike water permeability (Lepech and $\mathrm{Li}$ 2009), the chloride ion permeability of ECC specimens is substantially influenced by the presence of microcracks. 


\subsubsection{Effects of self-healing}

Table 4.2 shows the changes in the RCPT results of ECC specimens pre-loaded up to $80 \%$ of their splitting tensile deformation capacities after 7, 28 and 90 days of initial aging for additional 7, 30, 60 and 90 days of further CW curing. Percentage changes in chloride ion penetrability results of pre-loaded ECCs subjected to further CW conditioning are provided in Figure 4.4. Table 4.2 and Figure 4.4 both clearly show that based on initial curing time, type of mineral admixture and further curing time, self-healing in terms of the improvements in RCPT results was visible in all mixtures with a minimum level of $20 \%$, which shows the efficacy of CW conditioning on crack sealing. For all mixture types, whether initially cured for 7, 28 or 90 days, self-healing was quite a bit faster after 30 days of CW conditioning than after just seven days. However, when a comparison was made between the self-healing rates of ECCs under different transport property testing procedures, sorptivity results dropped drastically after seven days of CW curing, but only after 30 days of CW curing for RCPT results. The reason why self-healing rates were lower in terms of sorptivity results compared to RCPT results after the first seven days of CW curing could be due to the completely different natures of the tests. As previously mentioned, RCPT is an electrochemical test significantly affected by a number of parameters. Despite its ease of use, it measures the movement of all ions rather than only chlorides. Voltage applied for ion movement generates heat, which can be substantially high and increase the overall results for less mature specimens. Moreover, the electrical conductance of the concrete is important for RCPT and can be changed drastically by any conducting material (alkalis, free carbon content and so on) of whose existence in pore solution is also dependent on the maturity of specimens. In the case of sorptivity tests, however, the greater improvements observed - especially for younger specimens - could be due to the initial dominance of the connected pores resulting in a larger sorptivity values until they are filled, after which disconnected pores dominate with lower sorption effects. Beyond 30 days of CW curing, selfhealing in terms of RCPT results started to lose intensity. For example, for seven-day-old ECC_L specimens, average RCPT results dropped from 8318 to 2475 C level after the first 30 days of CW curing, with slight decrements when curing time was prolonged up to 60 and 90 days; the same results were $907 \mathrm{C}$ and $511 \mathrm{C}$, respectively. The same was true for specimens which were initially cured longer, along with the rest of the mixtures incorporating different mineral admixtures. This trend was not surprising, and was found to be attributable to the availability of more anhydrous 
materials at early ages and reductions in initial crack volumes at later ages, which may have restricted the visibility of marked self-healing.

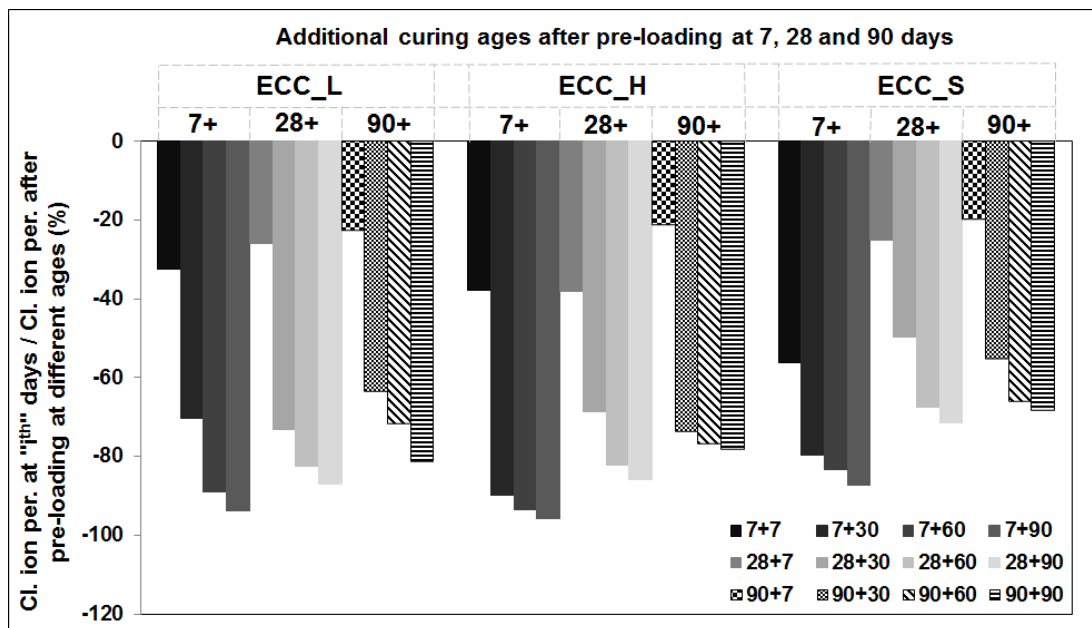

Figure 4.4: Percentage changes in chloride ion penetrability results of pre-loaded ECCs with further CW curing

Figure 4.4 shows the percentage reductions in RCPT results, indicating that initial curing time was one of the parameters influencing final healing results. Despite the variations in individual healing rates with respect to different mixture types, healing was more obvious for specimens cured for seven days. Contrary to what was expected, specimens that were initially cured for 28 and 90 days did not show noticeable differences in healing rates for all ECCs. For instance, 28-day-old ECC_L specimens that underwent an additional 30 days CW curing healed by 73\% of the initial RCPT value after pre-cracking, while the same mixture at 90 days healed by $63 \%$ under the same further curing conditions. It is important to note that this finding could answer the long-standing question of the effectiveness of late-age versus early-age self-healing in cementitious composites. However, further studies, including those examining specimens cured for longer periods, are needed for a complete clarification of this finding.

In regards to the influence of different mineral admixtures on RCPT results of pre-cracked ECC specimens, it can be generally stated that all of the mixtures showed healing rates close to each other, although in some cases ECC_S specimens showed slightly lower results. In a comparison of the self-healing rates of 28-day-old ECC_L and ECC_H specimens with ECC_S specimens after 30 days of CW curing, the results were $73 \%$ and $69 \%$ against $50 \%$ of the initial values after pre-loading, respectively. A similar modality held true for seven- and 90-day-old ECC_S 
specimens subjected to different periods of CW curing. Possible factors that could be decisive on lower healing rates in terms of the final chloride ion penetrability results of mixtures with slag particles have already been explained. Despite the fact that RCPT results varied significantly based on the chemical composition and initial curing ages of the mixtures, it should be stressed that 30 days of CW curing is adequate for most of the severely deteriorated ECCs to show low levels of chloride ion penetrability according to ASTM C 1202.

\subsection{Microstructure of self-healed ECC mixtures}

Scanning electron microscopy (SEM) was used to determine the autogenous healing products at the surface layer of the microcrack. Figure 4.5 compares SEM images (with EDX detector) of ECC samples at $90+90$ days of CW. It is apparent from the Figure that CW-cured samples of ECC mixes applied to different crack widths appear to be totally healed. Using EDX, the composition of surface chemicals was analysed which revealed that the microcracks of fly ash-ECC samples that were less than $50 \mu \mathrm{m}$ wide, were filled either partially or completely with a combination of calcite particles and calcium silicate hydrate gels. These findings are likely to be attributable to the continuous hydration and pozzolanic reaction of SCM. However, SEM verified that microcracks wider than $100 \mu \mathrm{m}$ in the slag-ECC specimens cured under CW were healed with considerable quantities of calcium carbonate deposition.

To better clarify final self-healing products, XRD measurements were performed on powder samples scratched with a razor along the healed crack lines of ECC specimens. Figure 4.6 presents XRD patterns obtained using an X-ray diffractometer with $\mathrm{Cu}$ Ka radiation and $2 \theta$ scanning, ranging between $10^{\circ}$ and $90^{\circ}$ of $2 \theta$. From Figure 4.6, quartz from silica sand and/or fly ash and some calcite were present in the diffractogram of the pre-loaded fly ash-ECC sample that healed. As Figure 4.6 demonstrates, the samples taken from pre-loaded ECC_L are comparable to those of ECC_H, though calcite peaks were higher in ECC_H. The diffractogram shows that the majority of the products in the ECC_S sample are calcite, although quartz is present. These findings signify that in the healing process of ECC_S, calcite is the predominant crystalline product present. Calcium ions from the hydration products are presumed to seep out of the hardened matrix and into cracks to react with carbon dioxide; this enables the cracks to heal and promote the formation of calcium carbonate. Water is an essential requirement for the leaching process. Gaseous carbon dioxide will only react with $\mathrm{CH}$ in circumstances of a long duration and high relative humidity 
(Neville, 2002). In contrast to what has been observed in the controlled carbon dioxide settings of a laboratory, the concentration of carbon dioxide as a bicarbonate solution and as a gas is considerably increased by water or water cures (Lauer, 1956). The sustained hydration of unhydrated cement particles is also accelerated in water.
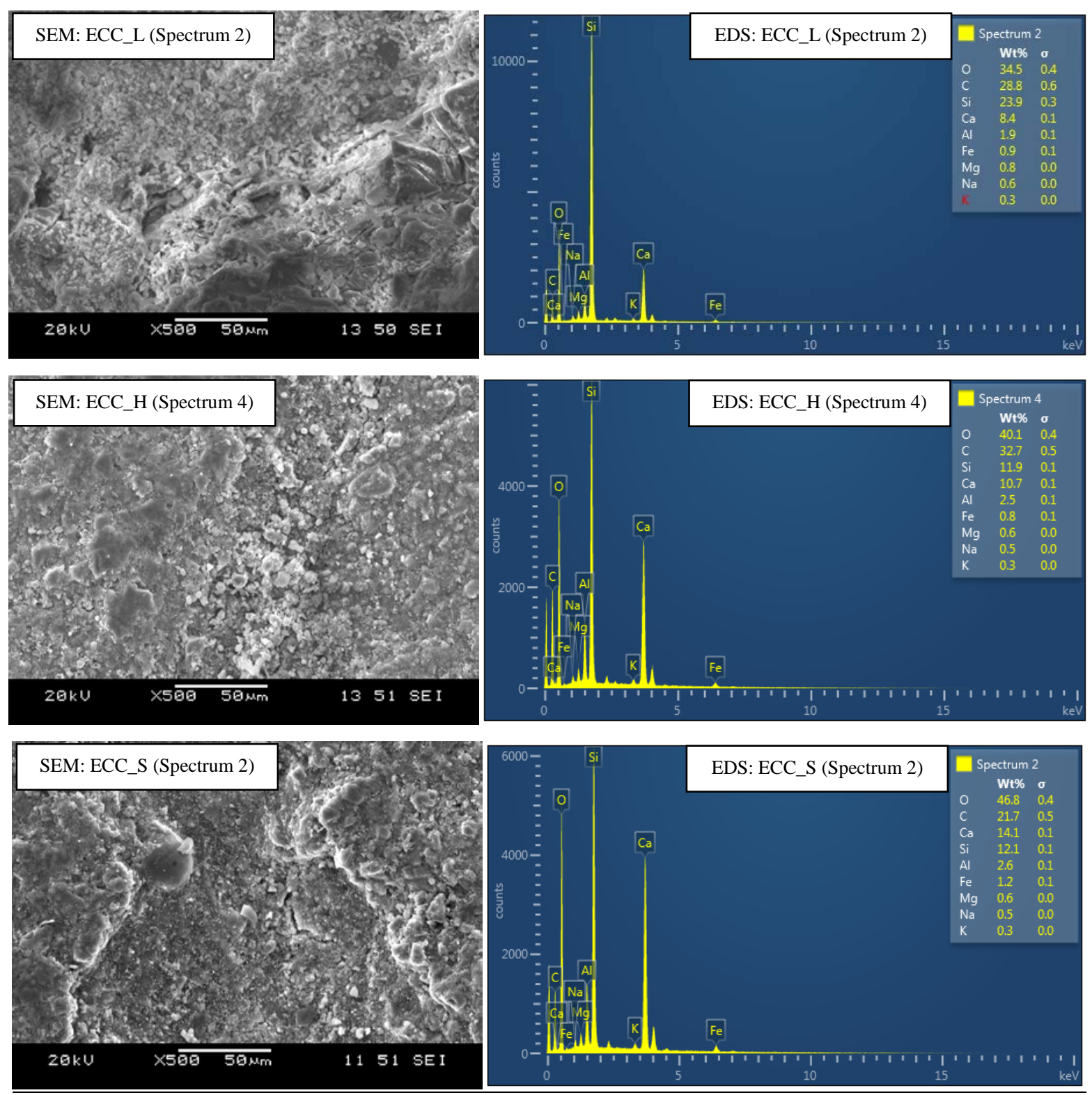

Figure 4.5: SEM micrograph with EDS pattern of products in self-healed cracks of the surface layer of ECC_L, ECC_H, and ECC_S 

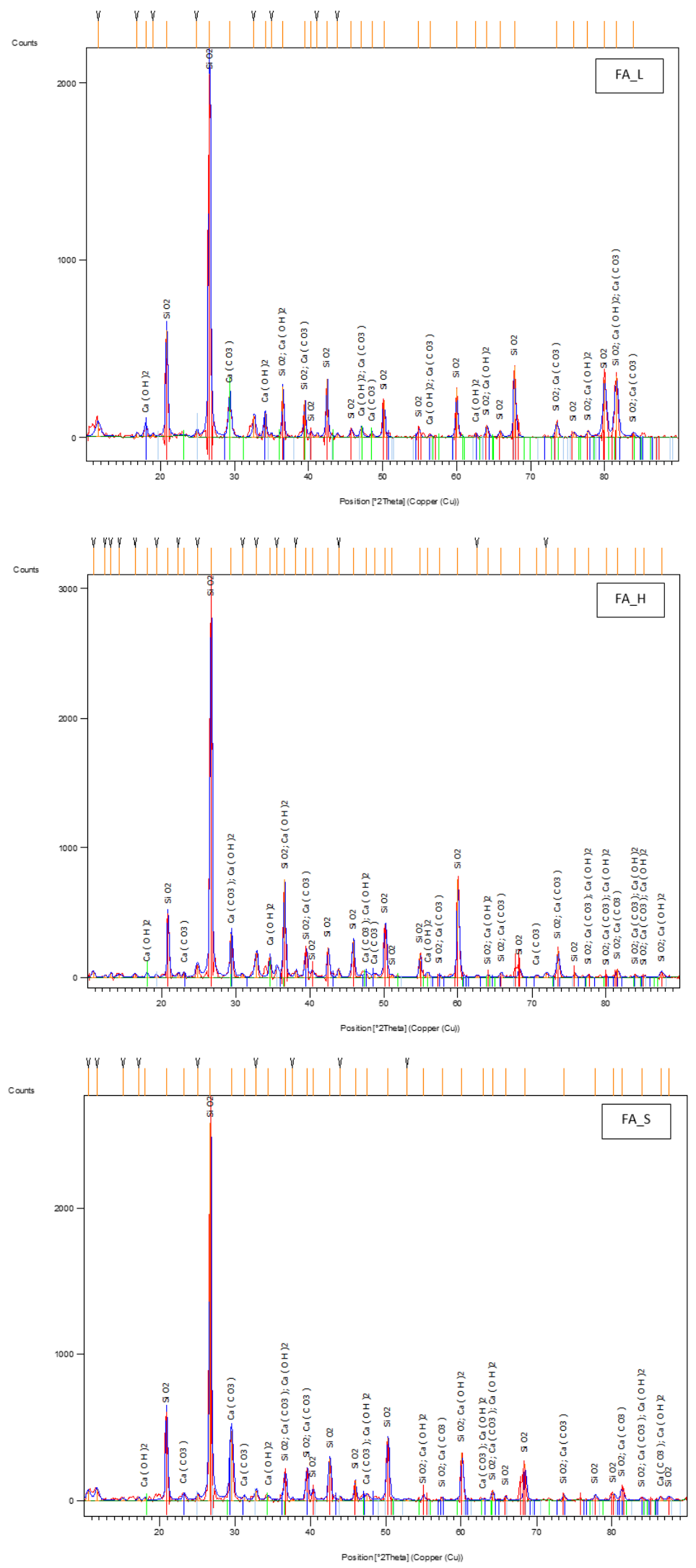

Figure 4.6: XRD patterns of self-healing products in ECC mixtures 
There may be a correlation between the high $\mathrm{pH}$ value of the pore solution and the superior autogenous healing capability of ECC_S samples. It is recognised that the chemical properties of the aqueous phase in cement paste is dominated by C-S-H gels combined with $\mathrm{CH}$ and alkalis, such as $\mathrm{Na}_{2} \mathrm{O}$ and $\mathrm{K}_{2} \mathrm{O}$; these are responsible for the pore solution's high $\mathrm{pH}$. From a starting state with the dissolution of alkalis, it is simple to reach pH 12 or greater (Anstice et al., 2005). Yet this hyper-alkaline state is temporary; the expectation is that the $\mathrm{pH}$ value decreases as hydroxides are depleted over time. Controlling the compounds presented in Figure 4.6 and Table 3.1 offers a paradigm to understand the depletion of hydroxides that is attributed to the pozzolanic capacity of supplementary cementitious materials. Pozzolanic activity is strongly associated with silicon dioxide and as Table 3.1 and Figure 4.6 indicate, this is greater in fly ash than slag. However, calcium oxide, which is responsible for cementing behaviour, is higher in slag than fly ash. The dissolution of carbonate species is closely linked to falls in $\mathrm{pH}$, leading to the precipitation of calcite crystals. High levels of silicon dioxide are also associated with late-age autogenic healing behaviour that in the instance of ECC_H and ECC_L samples use more CH (Edvardsen, 1999). Inversely to the low levels of silicon dioxide in slag, the level of CH in ECC_S samples is predicted to be high and it is feasible that the high $\mathrm{pH}$ value was maintained, raising the precipitation rates of calcium carbonate (Edvardsen, 1999). This, in turn, promotes autogenic healing.

\subsection{Chapter summary}

This chapter presented the evaluation of the self-healing mechanism in cementitious composites comprised of mineral admixtures greatly differing in chemical composition. During the analysis, concentration was placed mainly upon the assessment of the mechanism in terms of sorptivity and RCPT measurements. 


\section{Chapter 5}

\section{Assessing the Self-Healing Capability of Cementitious Composites under Progressively Increasing Mechanical Loading}

\subsection{Introduction}

This chapter investigated the effects of progressively increasing sustained loading on self-healing behavior of 180-day-old microcracked Engineered Cementitious Composites (ECC) incorporating different mineral admixtures (MAs). After introducing microcracks to the specimens with applied severe pre-loading, some of them were subjected to progressively increasing sustained loading. All the specimens were then subjected to continuous moist curing for 150 days to evaluate selfhealing performance. Mechanical property such as modulus of rupture (MOR) and mid-span beam deflection characterizations and ultrasonic pulse velocity (UPV) measurements were used to assess self-healing capability.

\subsection{Experimental Program}

\subsubsection{Materials, mixture proportions and basic mechanical properties}

Four different ECC mixtures incorporating three different types of fly ash (two Class-F and one Class-C) and ground granulated blast furnace slag (S, [ECC_S]) were produced. Although two of the fly ash types were selected to be Class-F in accordance with ASTM C 618, denomination was made according to the lime content in mixtures incorporating fly ash (i.e. low calcium (FA-L, [ECC_L]), medium calcium (FA-M, [ECC_M]) and high calcium (FA-H, [ECC_H])). In addition to the four mineral admixtures (MA), ordinary Portland cement (PC), silica sand with maximum aggregate size of $400 \mu \mathrm{m}$ and absorption capacity of $0.3 \%$, water, poly-vinyl alcohol (PVA) fibers with a diameter of $39 \mu \mathrm{m}$, nominal tensile strength of $1610 \mathrm{MPa}$ and specific gravity of 1.3 and high range water reducing admixture (HRWRA) were used in the production of mixtures. Table 3.1 presents the chemical and physical properties of Portland cement and the different mineral admixtures. ECC mixtures were produced with a water to cementitious materials (PC+MA) ratio (W/CM) of 0.27 , and mineral admixture to Portland cement ratio (MA/PC) of 1.2, by mass. Mixture proportions are listed in Table 3.2. 
Table 5.1: Mechanical Properties of ECC mixtures

\begin{tabular}{lccccc}
\hline Mechanical Properties & & \multicolumn{4}{c}{ Mix ID. } \\
\cline { 3 - 6 } & & ECC_L & ECC_M & ECC_H & ECC_S \\
\hline Compressive strength (MPa) & 28 days & 55.5 & 54.7 & 58.7 & 77.2 \\
& 90 days & 66.2 & 69.5 & 61.9 & 81.5 \\
& 180 days & 72.6 & 73.4 & 69.8 & 84.3 \\
\hline Flexural & 28 days & 10.2 & 9.9 & 11.6 & 11.5 \\
strength (MPa) & 90 days & 10.7 & 10.6 & 11.7 & 11.9 \\
& 180 days & 10.8 & 10.9 & 11.6 & 12.1 \\
\hline Flexural & 28 days & 4.4 & 3.8 & 3.1 & 2.4 \\
deformation (mm) & 90 days & 4.2 & 3.2 & 3.0 & 2.4 \\
& 180 days & 4.1 & 3.1 & 2.9 & 2.2 \\
\hline
\end{tabular}

The basic mechanical properties of the ECC mixtures were assessed considering the flexural parameters under four-point bending load (flexural strength and mid-span beam deflection) and the compressive strength results of 28,90 and 180-day-old $360 \times 75 \times 50 \mathrm{~mm}$ prism (length $\times$ width $\times$ depth) and $50 \mathrm{~mm}$ cubic specimens, respectively. After the completion of casting for mechanical property characterization, specimens were kept in molds for one day at $23 \pm 2{ }^{\circ} \mathrm{C}$, $50 \pm 5 \% \mathrm{RH}$ and then moved into plastic bags at $23 \pm 2{ }^{\circ} \mathrm{C}, 95 \pm 5 \% \mathrm{RH}$ for six days, followed by curing in a laboratory medium at $23 \pm 2{ }^{\circ} \mathrm{C}, 50 \pm 5 \% \mathrm{RH}$ until the end of 28,90 and 180 days. Mechanical property characterizations were made using the average of results obtained from eight different specimens; the results are shown in Table 5.1. As seen from Table 5.1, while the 28-dayold compressive strength results of FA-bearing ECCs were in the range of 55.0 and $59.0 \mathrm{MPa}$, ECC_S specimens reached 77.2 MPa, which is correlated with the higher fineness and cementing behavior of individual slag particles, resulting in a more compact microstructure. It is clear that the variation in flexural strength results of ECC mixtures was not as high as in compressive strength results, varying between 9.9 and $11.6 \mathrm{MPa}$ after 28 days. This behavior is related to the more elaborate material properties (e.g. tensile first cracking strength, ultimate tensile strength and tensile strain capacity) governing flexural strength results (Qian et al., 2009). When mid-span beam deflection results (which reflect the ductility of ECC materials) are considered, it can be seen that ECC_S mixture showed the lowest average flexural deformation at the end of 28 days (2.4 $\mathrm{mm}$ ), while ECC_L mixture was almost two times that of the ECC_S specimens, at $4.4 \mathrm{~mm}$. The increased ductility of ECC_L specimens was attributed to the tendency of low-calcium fly ash particles to reduce PVA fiber/matrix interface chemical bond and matrix toughness, while increasing the interface frictional bond in favor of attaining high tensile strain capacity (Wang and 
$\mathrm{Li}, 2007)$. When 90- and 180-day-old basic mechanical properties of ECCs are considered, it is evident that despite changes in the values compared to 28-day-old specimens, results did not show marked changes between individual curing ages. This implies that 90 days of curing was almost adequate for full maturity attainment.

\subsubsection{Sample preparation and pre-cracking}

As previously stated, the main purpose of this study was to investigate the effect of progressively increasing sustained loading on self-healing of microcracked ECCs. To do this, a number of prism specimens measuring $360 \times 75 \times 50 \mathrm{~mm}$ (length $\times$ width $\times$ depth) was produced from each mixture. After 24 hours inside the molds at $23 \pm 2{ }^{\circ} \mathrm{C}, 50 \pm 5 \% \mathrm{RH}$, all of the specimens were removed and put inside plastic bags at $23 \pm 2{ }^{\circ} \mathrm{C}, 95 \pm 5 \%$ RH for 180 days. A 180-day curing period was selected to make sure that specimens had completed most of their hydration reactions and reached a very mature state. As discussed previously, attainment of very high maturity after 180 days of initial curing can clearly be monitored from mechanical property characterization. For observation of self-healing in damaged ECCs, microcracks were produced in the specimens using four-point bending loading in a controlled manner at the age of 180 days. Since it is not easy to create similar microcracking damage on ECC mixtures incorporating MAs with greatly varying chemical compositions, initial pre-loading level of specimens was selected to be $75 \%$ of the mid-span beam deflection values at 180 days. It should be noted that 180-day-old average mid-span beam deflection results of at least six different specimens from ECC_L, ECC_M, ECC_H and ECC_S mixtures were 4.1, 3.1, 2.9 and $2.2 \mathrm{~mm}$, respectively, as seen in Table 5.1. After the application of initial pre-loading, some of the pre-loaded beam specimens underwent progressively increasing sustained loading for an additional 150 days, while others did not. For the specimens kept in sustained loading device, after initial pre-loading the level of the first sustained loading was set to $40 \%$ of ultimate flexural load. After each 30-day period under water, sustained loading level was increased by $10 \%$ until the end of 120 days (80\%) (Figure 5.1). 


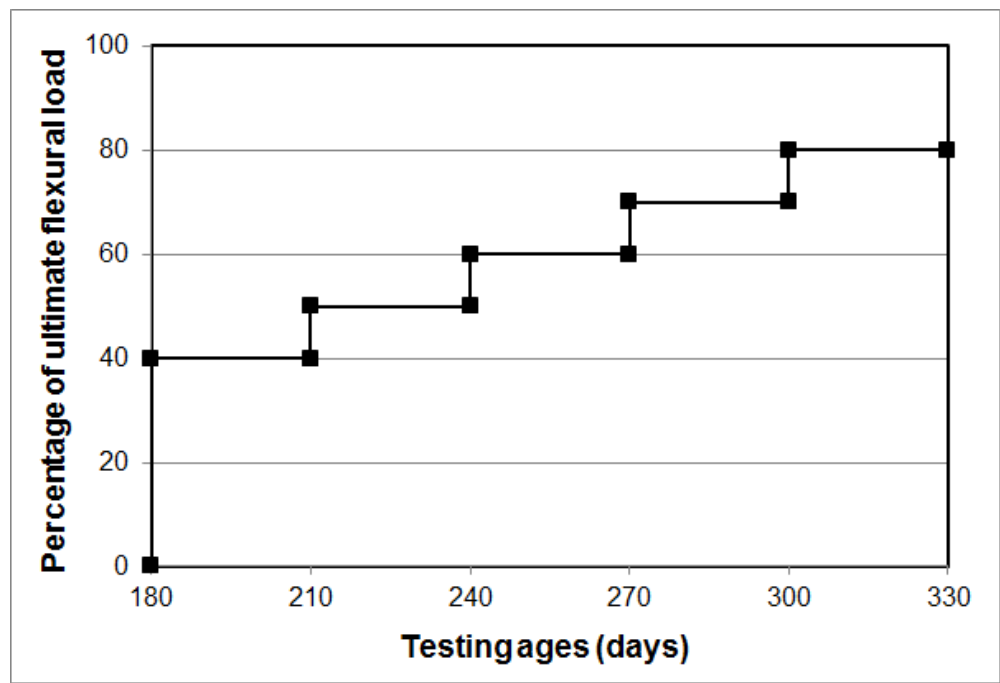

Figure 5.1: Changes in sustained loading level with time

A torque wrench was used to control periodic increments in sustained flexural loading level (Figure 5.2). A pair of steel rollers supported the beams and loading was applied with similar rollers placed over one third of the supporting span length. Specimens were loaded using threaded rods tightened by a torque wrench. To evaluate the load placed on the specimens, a load cell was initially inserted in place of the specimen and torque was applied with the torque wrench, which was then calibrated against the load cell. This calibration was determined via two separate trials before testing, with results shown in Figure 5.3. As seen, there is a linear relationship between the applied torque and the loading itself, and the results correspond well with each other. It should be mentioned that during each 30-day period of sustained loading, slight relaxations took place. However, these were accounted for in the curing periods of each sustained loading level. Along with the pre-loaded specimens, some beam specimens without pre-loading (sound) were kept for comparison. To evaluate self-healing, longitudinal ultrasonic pulse velocity (UPV) measurements were performed on specimens with and without progressively increasing sustained loading after each 30-day period of water exposure, according to ASTM C 597. Moreover, mechanical properties (modulus of rupture [MOR] and mid-span beam deflection) of ECCs with and without sustained loading were investigated before and after 150 days of moist curing. 


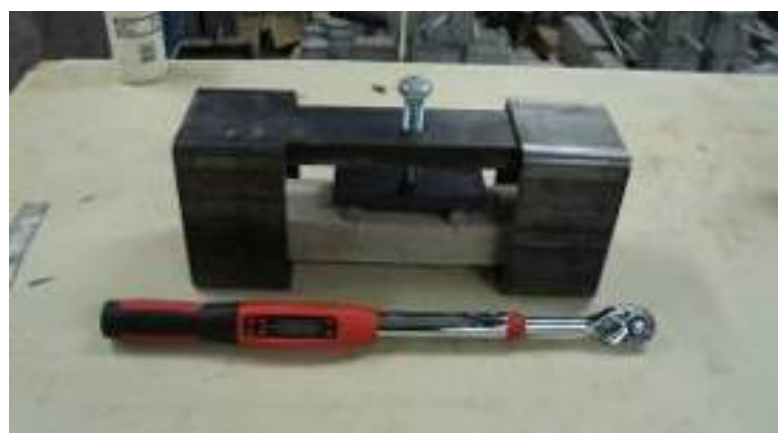

(a)

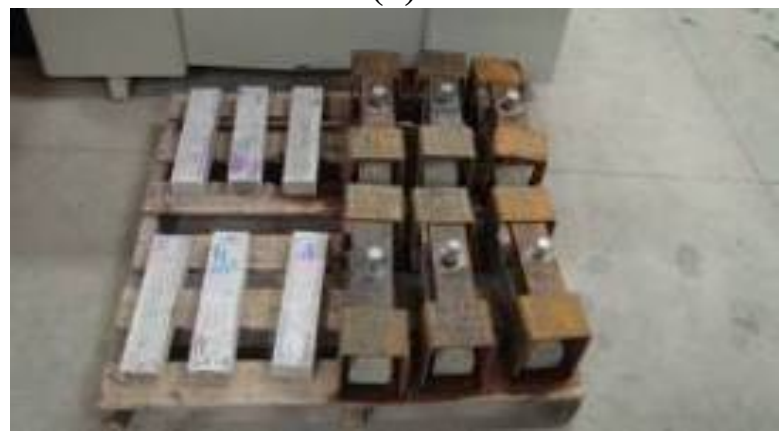

(c)

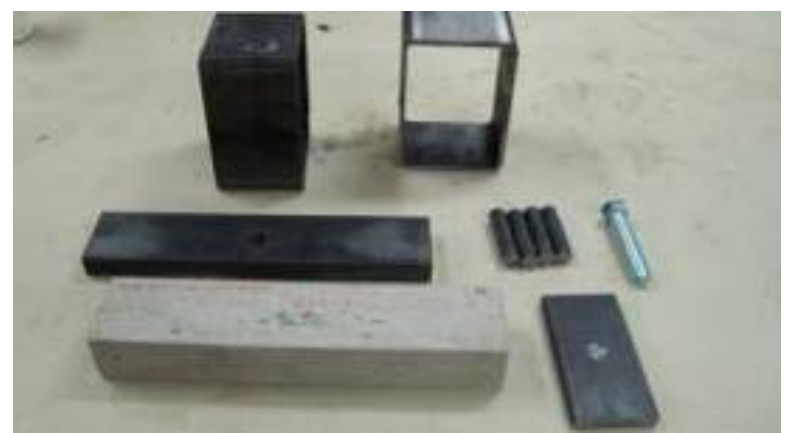

(b)

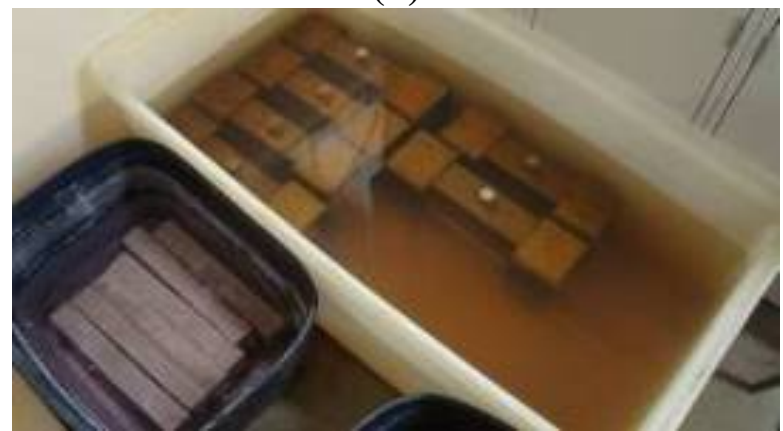

Figure 5.2: Torque wrench, application of sustained loading and curing of specimens

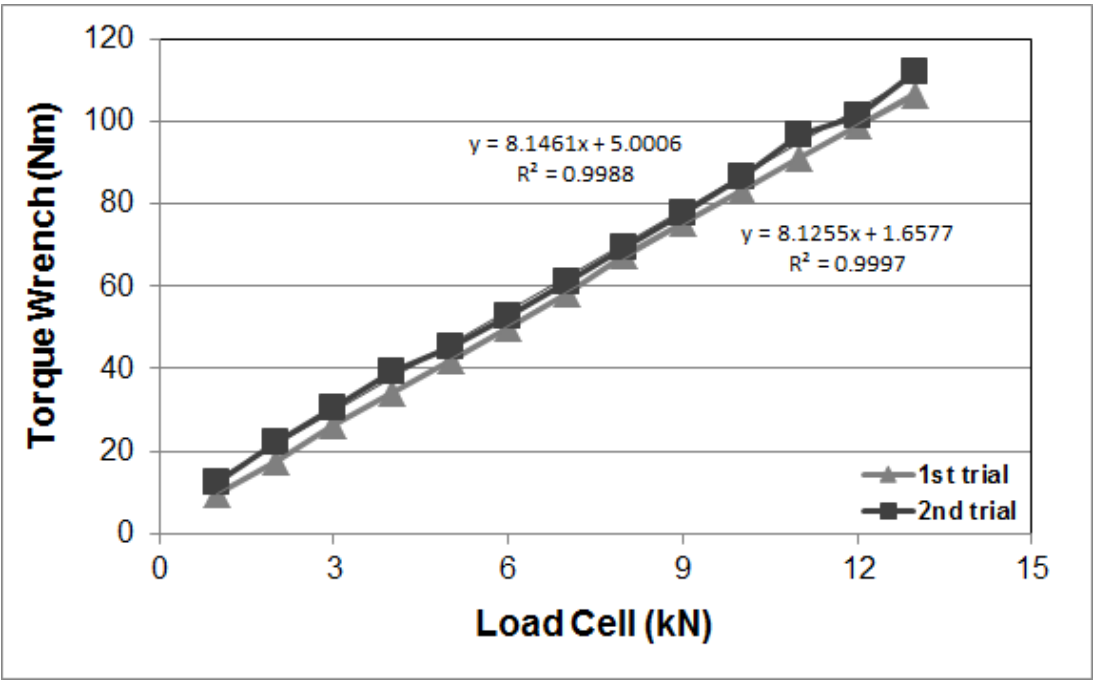

Figure 5.3: Calibration of torque wrench against the load cell two weeks before the start of actual tests

\subsection{Results and discussion}

\subsubsection{Recovery of mechanical properties}

\subsubsection{Modulus of rupture (MOR)}

Modulus of rupture (MOR) results of ECC specimens, calculated by using the maximum flexural loads obtained from load-deflection curves, are displayed in Table 5.2. All the values presented in 
this table were calculated by averaging at least eight different beams tested under four-point bending loading. The table includes the results of sound ECC specimens tested up to failure after 180 days of initial curing. After 150 days of further moist curing with specimens pre-loaded up to $75 \%$ of their mid-span beam deflection capacities on their 180th days, they were released and reloaded up to failure. Moreover, the effect of progressively increasing sustained loading on selfhealing capability was monitored in specimens pre-loaded up to $75 \%$ of their mid-span beam deflection capacities after 180 days of initial curing and cured for 150 days under water, with or without progressively increasing sustained loading. Considering the 180-day MOR results of sound ECCs with different MAs, it can be clearly stated that results were close to each other, ranging between 11.1 and 12.1 MPa levels. Despite the minor differences in results, the highest average MOR values were acquired from the ECC_S specimens, while the lowest were from ECC_L. This finding regarding the results of mixtures incorporating different MAs is believed to be in strict connection with the previously discussed factors affecting compressive strength.

Table 5.2: Flexural properties of ECC mixtures before and after continuous water exposure

\begin{tabular}{|c|c|c|c|c|c|c|c|c|c|c|}
\hline \multirow{3}{*}{ Mix ID. } & \multirow{2}{*}{\multicolumn{2}{|c|}{$\begin{array}{l}\text { Sound specimens } \\
\text { tested up to } \\
\text { failure at } 180 \text { days }\end{array}$}} & \multirow{2}{*}{\multicolumn{2}{|c|}{$\begin{array}{l}\text { Sound specimens } \\
\text { tested up to } \\
\text { failure at } 330 \text { days }\end{array}$}} & \multirow{2}{*}{\multicolumn{2}{|c|}{$\begin{array}{l}\text { 180-day-old sound } \\
\text { specimens pre- } \\
\text { loaded, released } \\
\text { and re-loaded until } \\
\text { failure }\end{array}$}} & \multicolumn{4}{|c|}{$\begin{array}{c}\text { Specimens pre-loaded after } \\
180 \text { days and exposed to } \\
150 \text { days of continuous } \\
\text { moist curing }\end{array}$} \\
\hline & & & & & & & \multicolumn{2}{|c|}{$\begin{array}{c}\text { without } \\
\text { sustained loading }\end{array}$} & \multicolumn{2}{|c|}{$\begin{array}{c}\text { with } \\
\text { sustained loading }\end{array}$} \\
\hline & $\begin{array}{l}\text { MOR } \\
\text { (MPa) }\end{array}$ & $\begin{array}{l}\text { Deflection } \\
\text { (mm) }\end{array}$ & $\begin{array}{l}\text { MOR } \\
\text { (MPa) }\end{array}$ & $\begin{array}{l}\text { Deflection } \\
\text { (mm) }\end{array}$ & $\begin{array}{l}\text { MOR } \\
\text { (MPa) }\end{array}$ & $\begin{array}{l}\text { Deflection } \\
\text { (mm) }\end{array}$ & $\begin{array}{l}\text { MOR } \\
\text { (MPa) }\end{array}$ & $\begin{array}{l}\text { Deflection } \\
\text { (mm) }\end{array}$ & $\begin{array}{l}\text { MOR } \\
\text { (MPa) }\end{array}$ & $\begin{array}{l}\text { Deflection } \\
\text { (mm) }\end{array}$ \\
\hline ECC_L & 11.1 & 4.1 & 11.4 & 3.7 & 10.4 & 2.6 & 11.2 & 3.8 & 11.0 & 3.5 \\
\hline ECC_M & 11.2 & 3.1 & 11.6 & 3.0 & 9.9 & 2.0 & 10.8 & 2.7 & 10.6 & 2.4 \\
\hline ECC_H & 11.8 & 2.9 & 12.0 & 2.7 & 9.3 & 1.8 & 11.1 & 2.3 & 10.3 & 1.9 \\
\hline ECC_S & 12.1 & 2.2 & 12.2 & 2.1 & 9.0 & 1.5 & 11.2 & 2.0 & 10.0 & 1.6 \\
\hline
\end{tabular}

When focus is placed on the effect of pre-loading on MOR results, it is clear that pre-loading up to $75 \%$ of the mid-span beam deflection capacities of specimens resulted in decrements that differed from the initial values by 6.3\%, 11.6\%, 23.7\%, and 25.6\% in ECC_L, ECC_M, ECC_H and ECC_S mixtures, respectively. These findings show that the effectiveness of pre-loading in lowering MOR results varied and that the ductility of different mixtures was a decisive parameter in damage tolerance. To put it differently, initial pre-loading was less harmful in terms of MOR results for specimens with higher ductility. This was an anticipated outcome since some amount of loss in load-carrying capacity is inevitable upon pre-loading, regardless of MA type. In addition, cracks re-open from the same place with load application, despite significant crack closure upon load removal. However, one important point requiring further attention is that after pre-loading, 
minimum MOR results were achieved by ECC_S (9.0 MPa) specimens while the maximum results were obtained in ECC_L (10.4 MPa), which differs from the results of sound specimens tested up to failure with no initial pre-loading. This result suggests that slag-incorporating ECCs are more sensitive to pre-loading compared to those with fly ash (especially class-F fly ash), resulting in less recovery upon removal of the existing load. As suggested above, this can be related to the reduced sensitivity of mixtures with lower deflection capacity (i.e. ductility) against loading. However, it is important to note that although all of these specimens almost failed with initial preloading up to 75\% deflection capacity, they were able to recover most of their flexural strength values in the unloaded state, with the minimum average value reaching 9.0 $\mathrm{MPa}$ at the age of 180 days.

Although the effect was rather restricted, there were increments in the MOR results of 180-dayold sound specimens as they were aged for an additional 150 days under water. As indicated in Table 5.2, increases in MOR results were less pronounced in slag-bearing ECCs compared to those produced with fly ash (especially class-F fly ash). This behavior was due to the fact that 180 days of initial curing was more than enough for almost complete stabilization of hydration reactions of highly reactive slag-containing specimens, and the additional 150 days of moist curing did not make a noticeable difference. On the other hand, fly ash particles were more likely to contribute to the improved MOR results, even after 330 days of complete curing due to the highly pozzolanic behavior of fly ash compared to slag, which manifested itself more in later ages.

Considering the effect of self-healing on specimens subjected to pre-loading up to $75 \%$ of their mid-span beam deflection values after 180 days and kept without sustained loading for further 150 days under water, one can see that recoveries resulting in values close or even slightly higher than that of those sound specimens are achievable through proper selection of MA type. For example, while MOR results of sound ECC_L, ECC_M, ECC_H and ECC_S specimens were 11.1, 11.2, 11.8 and 12.1 MPa after 180 days of curing, initial pre-loading and subsequent 150 days of water exposure resulted in final values of 11.2, 10.8, 11.1 and 11.2 MPa, respectively. This indicates the efficacy of self-healing on MOR results with no regard to MA type. These results show that after 150 days under water, the ECC_L specimens attained average results greater than the sound specimens. Moreover, the ECC_M specimens exhibited the second-highest recovery ratio compared to sound specimens. In the case of the ECC_S mixture however, pre-loaded specimens 
showed the lowest recovery amount in comparison to the sound specimens. These results therefore suggest that final self-healing products of ECCs with class-F fly ash were stronger than ECC_S specimens, considering the recovery of MOR results. Additionally, these findings offer an insight into the nature of final self-healing products, which were also visually monitored on the crack faces of different specimens.
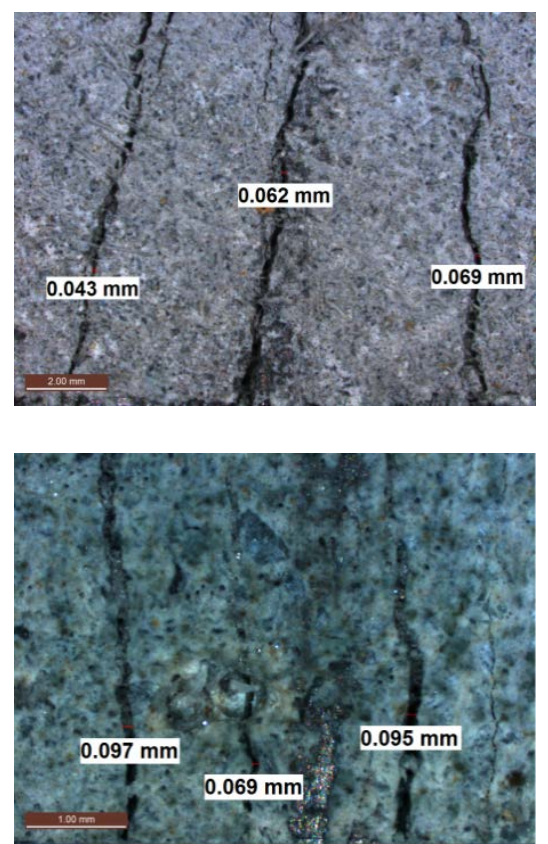

(a) 180 days

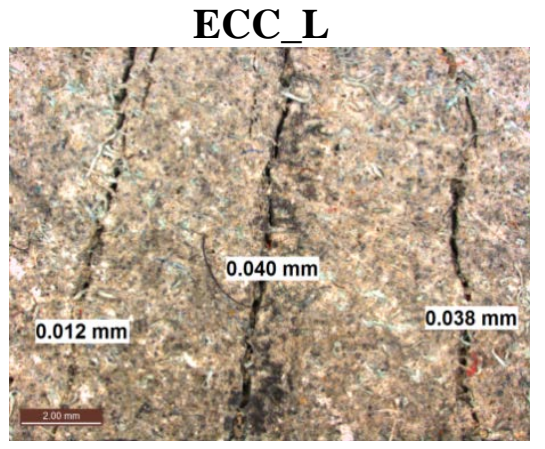

ECC_S

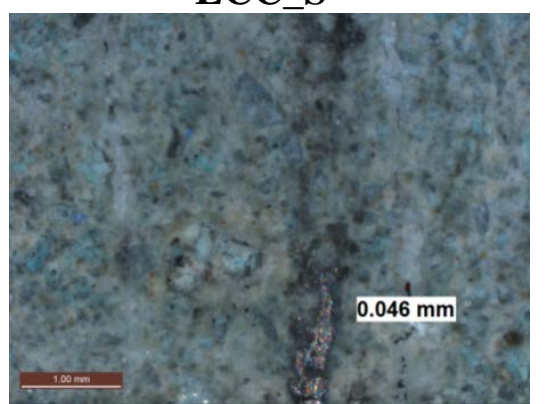

(b) 180+30 days
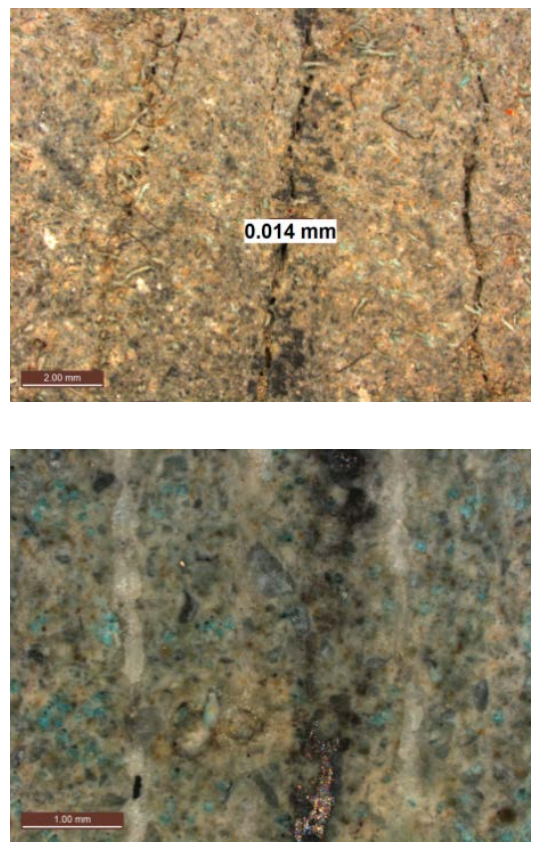

(c) $180+90$ days

Figure 5.4: View of microcracked surfaces after exposure to continuous moist curing

Figure 5.4 shows that after a certain amount of continuous water exposure of pre-loaded specimens, crack faces of ECC_S specimens were covered with traces of calcite, which manifested itself as white residue. This was not the case in specimens with fly ash, which suggests that furtherformed C-S-H gels were the dominant self-healing products, and are considerably stronger than calcite (Sahmaran et al., 2013). Overall, these findings could be the reasons why higher MOR recovery rates were obtained from ECCs with fly ash than from those with slag.

The effect of progressively increasing sustained loading on the recovery of MOR results was another point requiring further inspection. As seen in Table 5.2, the application of sustained loading, with intensity increased by $10 \%$ of ultimate flexural load after each 30 -day period until the end of 120 days, slightly lowered MOR results in comparison to pre-loaded specimens not subjected to sustained loading. For instance, 330-day MOR results of ECC_H mixtures were 11.1 
MPa when no sustained loading was applied. However, under progressive sustained loading, the result decreased to 10.3 MPa. This trend was valid for all mixtures produced in this study, and it was anticipated due to re-opening of microcracks after increments of sustained loading levels at 30-day intervals. However, it must be emphasized that with careful selection of MA, almost complete recovery of MOR results (according to sound specimens) is possible, even after severe initial pre-loading and subsequent exacerbation. It should be noted that despite having slightly lower recovery rates than specimens without sustained loading, the values obtained from specimens with sustained loading are still close to original values (180-day sound specimens), and higher than the specimens that failed directly after initial pre-loading. Like the specimens without sustained loading, ECC_S specimens showed the lowest recovery in MOR results compared to ECCs with fly ash (especially class-F fly ash [ECC_L and ECC_M]) when they were subjected to progressive sustained loading. Probable explanations for ECC_S behavior could be the very limited pozzolanic capacity of slag particles, which reduces the chance for self-healing as time passes, the tendency for slag-incorporating specimens to exhibit cracks with larger widths due to enhanced matrix fracture toughness and chemical bonding between PVA fibers and matrices (Sahmaran et al., 2012), and the lower capability of newly formed calcite particles to withstand monthly increased sustained loading compared to C-S-H gels.

\subsubsection{Mid-span beam deflection}

Table 5.2 presents mid-span beam deflection results of different ECC mixtures. Flexural deflection results, which can also be regarded as ductility results, were calculated by using at least eight different beam specimens from each mixture. During the calculation of deflection results, values corresponding to maximum flexural load points on load-deflection curves of ECC specimens were taken into consideration, and residual flexural deflection from pre-loading was not included in overall results for more reasonable estimation. Attaining adequate ductility in an HPFRCC such as ECC is a major goal; these types of materials are generally designed for high ductility. Table 5.2 shows substantial differences in the deflection results based on different MA usage, with 180day results of sound specimens showing a variation between 2.2 and $4.1 \mathrm{~mm}$ levels. When sound specimens were further cured under water for 150 days, slight reductions in deflection results were monitored due to continuous improvement of matrix and fiber-to-matrix interface properties; the same results ranged between 2.1 and $3.7 \mathrm{~mm}$ levels after this time. Considering the results of sound 
specimens from both ages, ECC_S specimens displayed the lowest mid-span beam deflection levels, with ECC_L specimens showing the highest. Potential causes of this finding have been discussed in previous sections.

When initial pre-loading was applied to the specimens before sudden re-loading, there were significant decrements in the final deflection results. For example, while average deflection capacity of 180-day-old sound ECC_L specimens was $4.1 \mathrm{~mm}$, the value went down to $2.6 \mathrm{~mm}$ with applied pre-loading; this situation held true for rest of the mixtures. Moreover, decrement rates in deflection results were higher compared to MOR values. For instance, the rate of decrease in MOR values of ECC_L specimens was 6.3\% after pre-loading, although the rate increased to $36.6 \%$ in mid-span beam deflection results. This might be attributed to faster localization of already-introduced individual microcracks, which cause final failure without significantly sacrificing maximum flexural load upon re-loading.

Exposure to 150 days of moist curing after initial pre-loading without any sustained loading led to marked improvements in mid-span beam deflection results, no matter what type of MA was used. When a comparison is made between specimens subjected to pre-loading at the age of 180 days, then released and re-loaded up to failure, and those subjected to pre-loading and left to self-heal for 150 days under water without any sustained load application, recoveries up to $46.1 \%$ were observed with appropriate MA usage (from 2.6 to $3.8 \mathrm{~mm}$ in ECC_L specimens). Similar MOR results were observed in specimens produced with slag and class-C fly ash, with lower recovery rates compared to those incorporating class-F fly ash. Reasons for the differing behaviors of different mixtures have been discussed in previous sections. In addition to results of pre-loaded specimens, results obtained after conditioning were close to those of sound specimens directly tested up to failure after 330 days of initial curing. For example, average deflection results of 330day-old sound specimens after failure were 3.7, 3.0, 2.7 and 2.1 mm for ECC_L, ECC_M, ECC_H and ECC_S mixtures, and 3.8, 2.7, 2.3 and $2.0 \mathrm{~mm}$ for the same mixtures kept in water for 150 days after severe pre-loading. Considering the results of specimens subjected to progressively increasing sustained loading after initial pre-loading on the 180th day and moist curing for 150 days, deflection results showed minor reductions compared to specimens without sustained loading; values dropped to 3.5, 2.4, 1.9 and $1.6 \mathrm{~mm}$ for ECC_L, ECC_M, ECC_H and ECC_S mixtures, respectively. Overall, these findings were also in line with the behavior observed in the 
MOR results. Despite slight decrements with applied progressive sustained loading, even the lowest deflection values (1.6 mm in ECC_S specimens) acquired under progressive sustained loading were still more than 100 times that of conventional concrete, which has an ultimate midspan beam deflection value nearly $0.01 \mathrm{~mm}$.

\subsubsection{Recovery of ultrasonic pulse velocity (UPV) measurements}

\subsubsection{ECCs without sustained loading}

In addition to mechanical property measurements, longitudinal ultrasonic pulse velocity (UPV) tests were also performed, according to ASTM C 597 standard, to monitor the effect of self-healing on ECC specimens. UPV test results of different ECC mixtures are provided in Table 5.3, which shows results for specimens subjected to initial pre-loading up to $75 \%$ of their 180 -day-old midspan beam deflection capacities, and for specimens subjected to monthly increased sustained loading starting from 180th day until the end of 330th day after the initial pre-loading. Values were found by taking average readings of eight beam specimens from each mixture. Table 5.3 clearly shows differences in UPV test results of sound ECCs with the usage of different MAs. 180-day UPV results were calculated by averaging results of sound specimens tested with and without progressive sustained loading; the results were 3954, 4018, 4005 and $4253 \mathrm{~m} / \mathrm{s}$ for ECC_L, ECC_M, ECC_H and ECC_S mixtures, respectively. These results indicate a close relationship with enhanced matrix maturity and high UPV results. Since relatively fine and reactive slag particles are more effective in modifying paste properties in favor of attaining more compact microstructure, higher UPV results are more likely to be acquired from these specimens.

With the application of initial pre-loading, decrements in UPV results occurred regardless of MA type. For example, as specimens were initially pre-loaded, UPV results decreased by an average of 2.4\%, 3.6\%, 3.8\% and 6.0\% in sound specimens for ECC_L, ECC_M, ECC_H and ECC_S mixtures, respectively. These results show that with the application of pre-loading, the highest decrements were obtained from ECC_S specimens in terms of UPV results as well. This finding has been previously discussed and attributed to the beneficial effects of slag on improved matrix maturity results, which in turn, increased the chemical bonding between fibers and matrix and reduced the formation of cracks with narrower widths. Although, all of the specimens that were subjected to initial pre-loading were almost failed under four-point bending loading, percent 
changes in UPV results with the applied initial pre-loading were minimal and do not seem to show big variations especially in the case of fly ash incorporating specimens. This result can be due to the similarities in chemical compositions of different MAs, as well as the fact that UPV tests are not sensitive enough to detect changes in specimens after the introduction of microcracks. Moreover, it seems that UPV results are closely related to average crack width measurements, so in specimens with wider crack widths (e.g. ECC_S), the drop in UPV results is greater. It is important to note that when considering average crack widths measured in different ECCs of the same age, ECC_S specimens showed the largest crack formation with 110-70 $\mu$ m levels, while the same values were near 80-30 $\mu \mathrm{m}$ for ECCs with different types of fly ash after 180 days of initial curing.

Table 5.3: Ultrasonic pulse velocity (UPV) test results of ECC specimens (units in m/s)

\begin{tabular}{|c|c|c|c|c|c|c|c|c|c|c|c|c|c|c|}
\hline \multirow{3}{*}{ Mix ID. } & & \multicolumn{3}{|c|}{$180 \mathrm{~d}}$. & \multicolumn{2}{|c|}{$180+30 \mathrm{~d}}$. & \multicolumn{2}{|c|}{$180+60 \mathrm{~d}}$. & \multicolumn{2}{|c|}{$180+90 \mathrm{~d}$} & \multicolumn{2}{|c|}{$180+120 \mathrm{~d}}$. & \multicolumn{2}{|c|}{$180+150 \mathrm{~d}}$. \\
\hline & & \multirow{3}{*}{$\frac{\text { Sound }}{3965}$} & \multirow{3}{*}{$\frac{\text { PL }^{*}}{3888}$} & \multirow{2}{*}{$\begin{array}{c}40 \%^{+} \\
\text {PL+SL } \\
\end{array}$} & \multirow{3}{*}{$\frac{\text { PL }}{4036}$} & $50 \%$ & \multirow[b]{2}{*}{ PL } & \multirow{2}{*}{$\begin{array}{c}60 \% \\
\text { PL+SL }\end{array}$} & \multicolumn{2}{|r|}{$70 \%$} & \multicolumn{2}{|r|}{$80 \%$} & \multirow[b]{2}{*}{ PL } & \multirow{2}{*}{$\begin{array}{c}- \\
\mathbf{P L}+\mathrm{SI}\end{array}$} \\
\hline & & & & & & $\mathbf{P L}+\mathbf{S L}$ & & & PL & $\mathbf{P L}+\mathbf{S L}$ & PL & $\mathbf{P L}+\mathbf{S L}$ & & \\
\hline \multirow{2}{*}{ ECC_L } & w/o SL & & & - & & - & 4058 & - & 4080 & - & 4114 & - & 4121 & - \\
\hline & with SL & 3943 & 3831 & 3763 & - & 4014 & - & 4022 & - & 4067 & - & 4063 & - & 4101 \\
\hline \multirow{2}{*}{ ECC_M } & w/o SL & 4004 & 3822 & - & 4098 & - & 4109 & - & 4131 & - & 4168 & - & 4192 & - \\
\hline & with SL & 4031 & 3922 & 3874 & - & 4001 & - & 4028 & - & 4093 & - & 4126 & - & 4179 \\
\hline \multirow{2}{*}{ ECC_H } & w/o SL & 4002 & 3841 & - & 4113 & - & 4194 & - & 4213 & - & 4256 & - & 4268 & - \\
\hline & with SL & 4008 & 3866 & 3835 & - & 4069 & - & 4140 & - & 4079 & - & 4206 & - & 4283 \\
\hline \multirow{2}{*}{ ECC_S } & w/o SL & 4264 & 3999 & - & 4357 & - & 4365 & - & 4352 & - & 4402 & - & 4423 & - \\
\hline & with SL & 4241 & 3996 & 3944 & - & 4286 & - & 4281 & - & 4332 & - & 4357 & - & 4430 \\
\hline
\end{tabular}

*Pre-loaded, †Pre-loaded \& sustained loading, ${ }^{+}$Numbers in percentages are the levels of sustained loading

In addition to the raw data in Table 5.3, Figure 5.5-a shows the percentage variations in UPV measurements of ECC mixtures not subjected to progressively increasing sustained loading after severe initial pre-loading. This figure also reflects the monthly self-healing performance of beam specimens in terms of UPV measurements. According to Figure 5.5-a and Table 5.3, one can easily conclude that there were improvements in UPV measurements with time, irrespective of MA used in ECC mixtures showing self-healing up to a certain extent. Taking ECC_S specimens as an example, average UPV results increased from 3999 to 4357 and 4423 m/s after 30 and 150 days of moist curing, respectively. In addition to the final UPV results after 330 days, values continuously increased after each 30-day interval and showed the formation of self-healing in microcracks. However, it should be noted that self-healing of microcracks upon exposure to continuous moist curing and continuing hydration reactions are not the only factors responsible for the increments in UPV results with time. Although the tests were started after 180 days of 
initial curing to reduce further hydration effect, the results were directly influenced by the integrity of the materials.

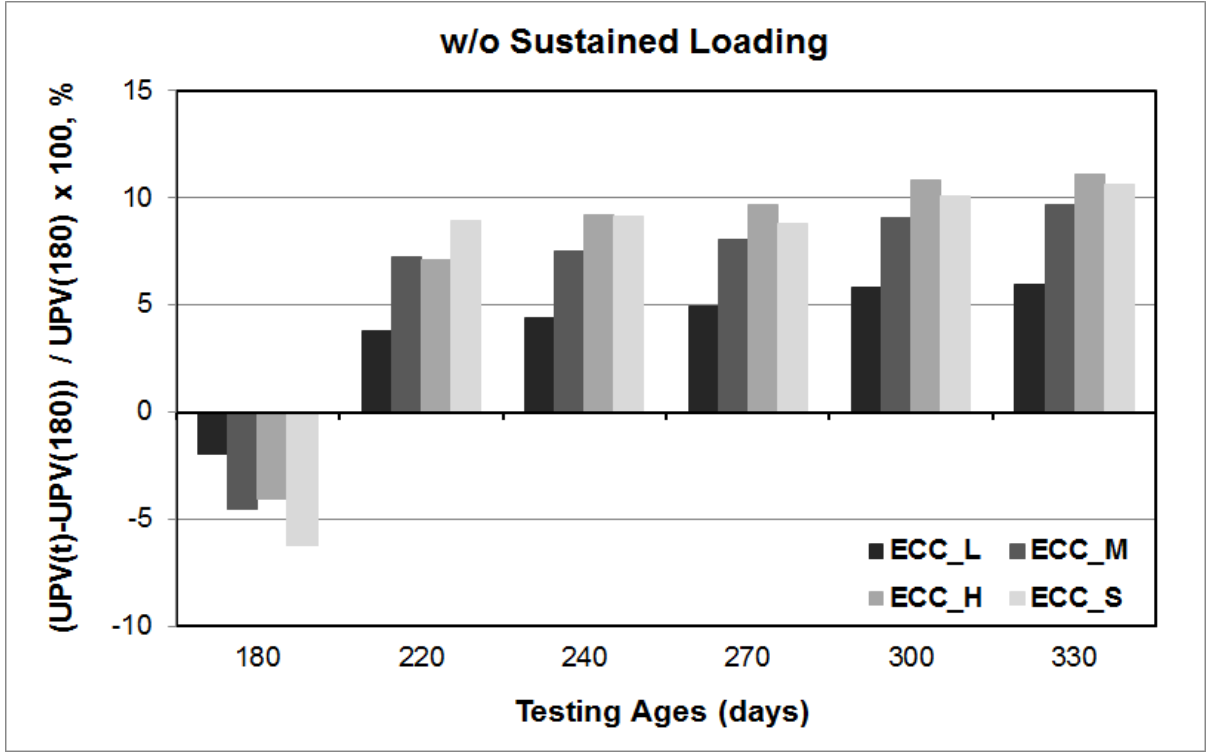

(a)

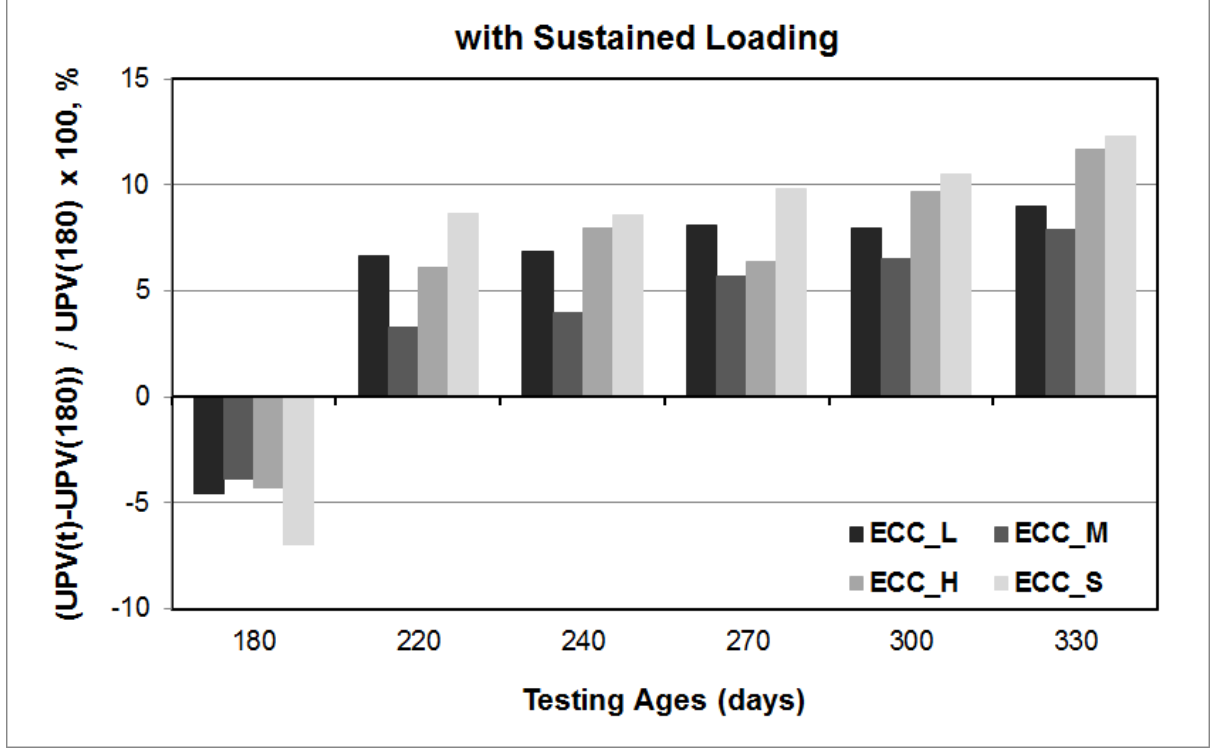

(b)

Figure 5.5: Percentage variations in UPV results of ECCs with the applied initial pre-loading and further curing a) without b) with progressively increasing sustained loading

Despite the varying changes in UPV results with further moist curing, 30 days under water was more than adequate for all ECC mixtures to attain UPV results greater than those of sound specimens. For example, UPV results of sound ECC specimens were 3965, 4004, 4002 and 4264 m/s after 180 days of initial curing for ECC_L, ECC_M, ECC_H and ECC_S specimens, while 
after severe initial pre-loading and 30 days of continuous moist curing, they were 4036, 4098, 4113 and $4357 \mathrm{~m} / \mathrm{s}$, respectively. In addition, most of the improvements in UPV results were achieved in the first 30 days of moist curing, although there was continuity to the end of the full 150-day period. This was most probably due to the fact that the anhydrous cementitious materials diminished significantly after 210 days of curing, lowering the chance for both crack healing and marked hydration. An increasing trend in UPV results was valid for all ECC mixtures, although the extent differed significantly depending on the type of MA selected. Figure 5.5-a shows a maximum $11.1 \%$ improvement in UPV results of initially pre-loaded specimens, depending on the age of curing and the MA used in the mixtures. Based on this figure, the higher UPV recovery results obtained from ECC_H and ECC_S mixtures in general had values that were comparable to each other at the end of each 30-day interval until the end of 150 days. The ECC_M and ECC_L mixtures followed in terms of improvements in UPV results with further curing. This result does not correspond with the recovery of mechanical property results, and was unexpected since the superior pozzolanic capacity of class-F fly ash particles is likely to contribute to the formation of self-healing and more mature paste, especially at later ages. In a different study, similar results showed the dominance of self-healing in ECCs with class-C fly ash and, in particular, slag (Sahmaran et al., 2013). That study reported that lower $\mathrm{SiO}_{2}$ amounts present - especially in the composition of slag particles - could lower the chance for pozzolanic reactions, thereby increasing the amount of portlandite and, relatedly, $\mathrm{pH}$ value of pore solution. When pore solution has higher $\mathrm{pH}$ values, carbonic acid (a product of reaction between water and $\mathrm{CO}_{2}$ ) can disassociate faster as bicarbonate $\left(\mathrm{HCO}^{3}-\right)$ and carbonate ions $\left(\mathrm{CO}_{3}{ }^{2-}\right)$, which are used in the formation of calcite by combining with $\mathrm{Ca}^{2+}$ ions leached away from C-S-H gels and portlandite in the presence of $\mathrm{CO}_{2}$ abundant water (Jooss, 2001; Edvardsen, 1999). Therefore, increased self-healing capability displayed by slag-incorporating ECCs was attributed to the formation of calcite up to a considerable extent, while specimens bearing Class- $C$ fly ash were influenced by both calcite and C-S-H gel formation (Sahmaran et al., 2013). The discrepancy in the self-healing behaviors of ECCs with different MAs is believed to be due to the varying natures of different tests. The precise detection of self-healing in terms of UPV measurements is not an easy task, due most probably to the lower sensitivity of test technique itself. This could be another possible reason behind the lower self-healing rates in terms of UPV results from ECC mixtures containing class-F fly ash. 180 days of initial aging is quite a long period for most pozzolanic reactions to take place, and thus 
pozzolanic capacity might not have been adequate to show itself worthy at the final stage of curing due to reduced precision of UPV testing. However, it is highly likely that it contributed substantially to the formation of the further C-S-H gels, which densified the matrices. As a concluding remark related to UPV improvement results of pre-loaded specimens, final UPV results of different mixtures were found to be very close to each other, although all the specimens showed self-healing performances with varying rates (Table 5.3).

\subsubsection{ECCs with progressively increasing sustained loading}

The effects of progressively increasing sustained flexural loading on self-healing capability of ECCs initially subjected to severe pre-loading up to $75 \%$ of their mid-span beam deflection capacities are shown as raw UPV data in Table 5.3 and as percentage changes in Figure 5.5-b. As in specimens with no sustained loading, initial pre-loading led to decreased UPV results in all 180day-old ECC mixtures. After initial pre-loading, 180-day-old beam specimens were put into the sustained loading device and further loaded until $40 \%$ of ultimate flexural loads were reached. As seen from Table 5.3, application of sustained loading, together with the initial pre-loading, caused UPV results to go down even more, although the effect was not as intense as in initial pre-loading. For instance, while a 5.8\% decrease took place in the UPV results after initial pre-loading of 180day-old sound ECC_S specimens, the rate increased up to 7.0\% with additional sustained loading. This behavior generally applied to mixtures with different fly ash types as well, which was expected due to further opening of already-introduced microcracks. From Figure 5.5-b, it can be concluded that comparable self-healing performances were acquired from specimens that were only subjected to initial pre-loading before moist curing, and those subjected to progressively increasing sustained loading after initial pre-loading. Moreover, the increasing trend in healing rates was mostly continuous, despite the fact that after each 30-day interval of moist curing, the torque wrench was adjusted to $10 \%$ more than the initial $40 \%$ until reaching $80 \%$ ultimate flexural load after 300 days. Taking the ECC_S mixture as an example, the healing rates after 30, 60, 90, 120 and 150 days of moist curing were $8.7 \%$, 8.5\%, 9.8\%, 10.5\% and 12.3\%, respectively. These healing rates were obtained when the sustained loading levels were 40\%, 50\%, 60\%, 70\% and 80\% of the ultimate flexural loads of ECC_S specimens. Enhanced self-healing performance in terms of UPV measurements was surprising in the presence of continuously increased sustained loading, since it was presumed that application of higher rates of sustained loading in every 30-day interval 
would overshadow the beneficial effects of self-healing in opposite crack faces. This might be explained by the initial crack opening factor and its possible effects on self-healing performance. When cracks with smaller openings were considered, self-healing was anticipated to occur at a slower rate due to space restriction for new self-healing products and slower diffusion of necessary species (i.e. water and $\mathrm{CO}_{2}$ ) into smaller cracks. When sustained loading level was increased however, cracks were likely to open more, leading to easier ingress of required substances for selfhealing to accelerate and providing more space for final healing products. Although some mixtures showed enhanced improvements in UPV recovery results, it can generally be stated that there were reductions in the healing rates of certain mixtures (ECC_M and ECC_H) when sustained loading was applied. However, it should not be overlooked that all of the mixtures with different MAs under the effect of progressively increasing sustained loading reached UPV results greater than those of sound specimens after only 30 days of water exposure. Throughout 150 days of complete curing, results did not show significant variations from each other. Overall this finding also provided an insight into how sensitive the UPV tests were to changes after self-healing.

\subsection{Chapter summary}

This Chapter discussed the effects of progressively increasing sustained loading on self-healing behavior of initially pre-loaded mature ECC specimens produced with different MAs with a wide variety of chemical compositions. During the experimental program, self-healing was evaluated with UPV measurements and mechanical property (modulus of rupture [MOR] and mid-span beam deflection) characterizations. 


\section{Chapter 6}

\section{Self-healing behavior of full-scale Cementitious Composites with different shear span to depth ratio}

\subsection{Introduction}

This Chapter presents the self-healing behavior of large scale ECC reinforced beams under load influence by giving priority to the individual effects of ultra-high ductility with moderate strength of ECC beams. To quantify the healing, flexural properties such as stiffness, deflection, ductility ratio and absorption capacity of the virgin, pre-loaded and re-loaded beams were examined before and after inducing the cracks.

\subsection{Experimental program}

\subsubsection{Pre-cracking and testing}

Twelve reinforced ECC beams were produced and tested. To evaluate the self-healing behavior of reinforced beam under shearing effect, ratios of shear span (a) to effective height or depth (d) were selected to be 'a/d' 1,2 and 3 . A low ratio of a/d between 1 and 3 was selected to monitor the behavior of test specimens under the influence of shear forces.

Four beams were prepared for each a/d ration. One beam was tested up to failure at the age of 28 days (ECC_V_28D) and another one was preloaded and reloaded at zero healing age (28 day-old) (ECC_PLRL_28D). For the remaining two beams of each a/d ratio, one beam was preloaded at the age of 28 days then left to heal under continuous water curing for 90 days before being reloaded to failure (ECC_RL_28+90D). The last/fourth beam was left as virgin beam (without preloading) and tested to failure at the end of 118 days (ECC_V_28+90D).

For each a/d ratio longitudinal tensile reinforcement of $2 \phi 16$ was used in the beams. To prevent local failure at the support points of reinforced beam specimens, a small number of shear reinforcements were used in these areas. No additional shear reinforcements were added along the beam length. Details are presented in Figure 6.1. Reinforcement properties were tested under uniaxial tension with five coupon specimens. Ribbed steel bars with $16 \mathrm{~mm}$ and $10 \mathrm{~mm}$ diameters were used as longitudinal and shear reinforcements over end supports, respectively. The yield 
strength (fsy), ultimate strength (fsu) and elastic modulus (E) of the $16 \mathrm{~mm}$ reinforcement were $520 \mathrm{MPa}, 625 \mathrm{MPa}$ and $205 \mathrm{GPa}$, respectively. The corresponding values for $10 \mathrm{~mm}$ reinforcement were $428 \mathrm{MPa}, 535 \mathrm{MPa}$ and $198 \mathrm{GPa}$, respectively.
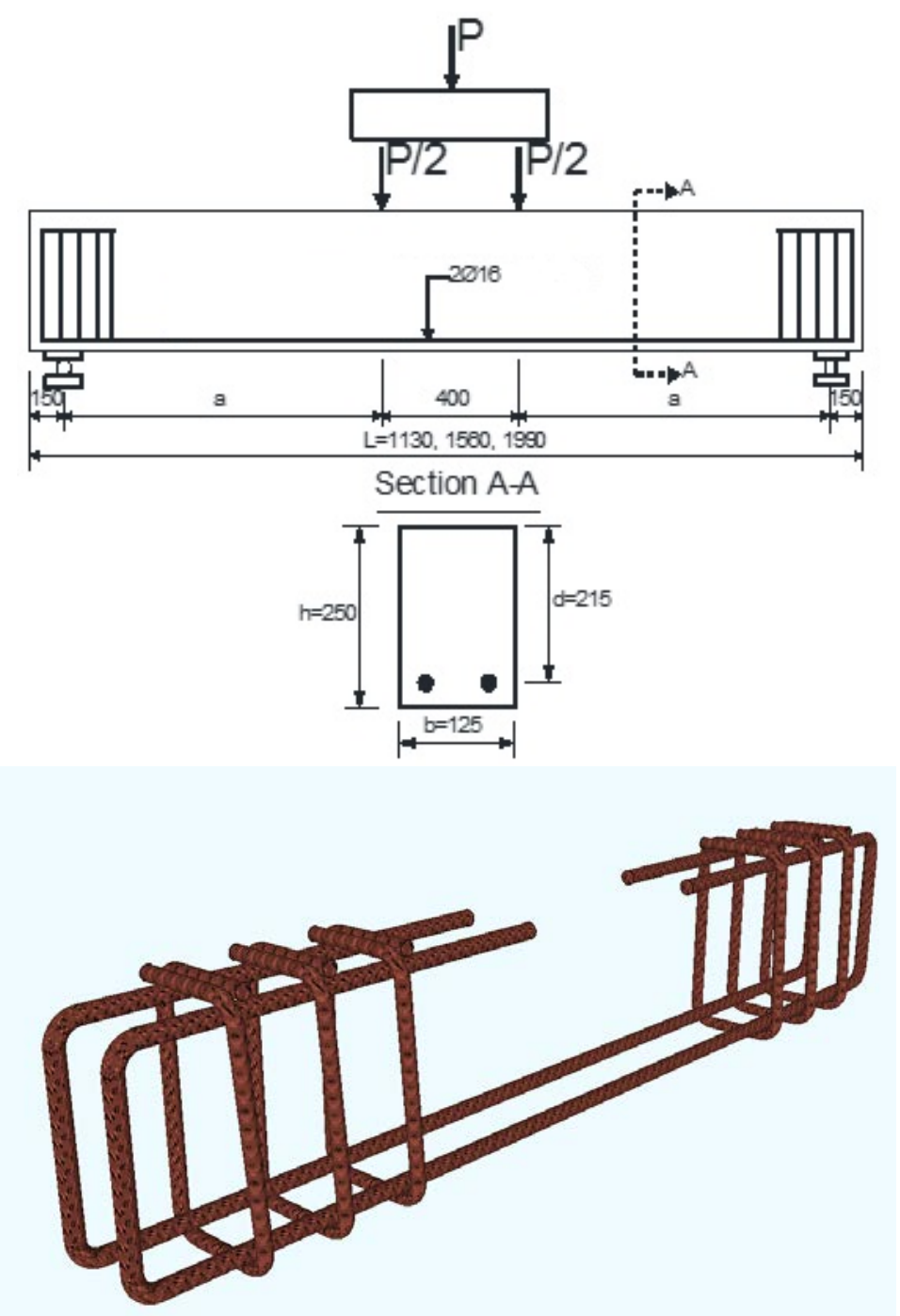

Figure 6.1: Reinforcement details of the specimens

For observation of self-healing in preloaded damaged ECC beams, microcracks were produced in the specimens using four-point bending loading in a controlled manner at the age of 28 days. Before the test, one beam from each a/d ratio was loaded up to failure under four-point bending loading for the determination of ultimate bending deformation capacities. $50 \%$ of the ultimate bending deformation capacity of ECC beams was set as a pre-cracking limit for all a/d ratios. After the application of initial pre-loading, the pre-loaded cracked beams were left to heal under continuous water curing for 90 days before tested to failure. 


\subsubsection{Material properties}

ECC mixtures used for all twelve test beam specimens were produced to have similar compressive strengths. The ECC mixture had a water to cementitious material ratio (W/CM) of 0.27 and a fly ash to Portland cement ratio (FA/PC) of 2.2, by mass. Components similar to those of typical fiberreinforced concrete were used for ECC production, including CEM I 42.5 type cement (similar to ASTM Type I), sand, Class-F FA with lime content of 5.57\%, water, fibers, and a high-range water-reducing admixture (HRWRA). ECC mix proportion details are presented in Table 6.1.

\begin{tabular}{lc}
\multicolumn{2}{l}{ Table 6.1: Mixture proportions and basic mechanical properties } \\
\hline Ingredients, $\mathbf{( k g / \mathbf { m } ^ { 3 } )}$ & ECC \\
\hline Portland cement & 375 \\
Fly ash & 823 \\
Water & 318 \\
Silica sand & 446 \\
PVA fiber & 26.0 \\
HRWRA & 2.3 \\
\hline Mechanical Properties (28-day) & \\
\hline Compressive strength (MPa) & 46.1 \\
Flexural strength (MPa) & 7.41 \\
Flexural deformation (mm) & 4.52 \\
\hline
\end{tabular}

To minimize matrix fracture toughness, which is necessary to obtain multiple microcracking and consequently strain-hardening behavior, smaller size aggregates are preferred. Therefore, silica sand with a maximum aggregate size of $1 \mathrm{~mm}$ was incorporated into the ECC mixtures. Fibers used in ECC were poly-vinyl alcohol (PVA) type with an average diameter of $39 \mu \mathrm{m}$, an average length of $8 \mathrm{~mm}$, and a tensile strength of $1610 \mathrm{MPa}$. HRWRA was added to the mixture until the desired fresh ECC characteristics (described in another study) were visually observed (Yang et al., 2009).

Six $\varnothing 100 \times 200 \mathrm{~mm}$ cylinder specimens were prepared for the determination of compressive strength of ECC. Flexural parameters (flexural strength and deflection) were evaluated with fourpoint bending tests, using six 400×100×75 mm beam specimens made of ECC mixture. Four-point bending tests were performed at a loading rate of $0.005 \mathrm{~mm} / \mathrm{s}$ using a universal testing machine. Typical bending test results are displayed in Figure 6.2 as flexural stress-deflection response for ECC at the age of 28 days. As seen from Table 6.1, mean compressive strength of ECC at 28 days 
was 46.1 MPa and mean flexural strength at 28 days was 7.41 MPa. Mean ultimate deflection capacity from the four-point bending test, which reflects the material ductility of ECC mixture, was $4.52 \mathrm{~mm}$ after 28 days.

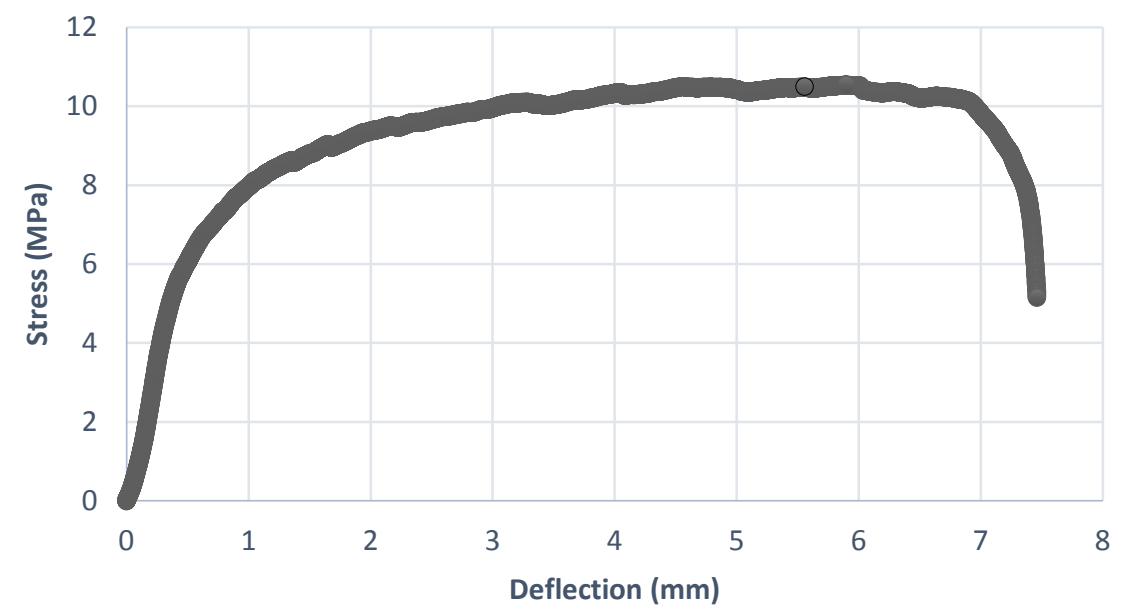

Figure 6.2: Typical flexural stress-deflection graphs of ECC after 28 days

\subsubsection{Test setup and instrumentations}

Four-point bending test setup was adopted to evaluate the behavior of reinforced ECC beams under shear loading. Shear force was applied monotonically to the specimens until failure in case of sound/virgin beams and up to $50 \%$ of the ultimate load in the case of pre-loaded beams. The midspan beam deflection, crack width and strain in tensile reinforcements were recorded through a computer data acquisition system. The test setup and locations of linear voltage displacement transducers (LVDTs) are shown in Figure 6.3.

For sound/virgin, pre-loaded and re-loaded (healed) beams, the load-deflection curves were plotted by considering the vertical deflection values recorded at the mid-span of the maximum moment region and three points on both symmetrical sides of the beams. Strength, stiffness, ductility ratios and energy absorption capacities of the specimens wereobtained from the load-deflection response. Strain measurements were recorded at the maximum moment regions for tensile reinforcement of each beam specimen. Strain gauges placed at the middle of each steel bar before casting are shown in Figure 6.4. 


\subsection{Experimental results and discussion}

\subsubsection{General behavior and failure modes of the virgin specimens at the age of 28 days}

Load-deflection curves of beams obtained from four-point bending loading tests are shown in Figure 6.5 and the results are summarized in Table 6.2Error! Reference source not found.. By using the load-deflection curves, general behavior, failure modes, strength, stiffness, ductility and energy absorption capacity of beam specimens were obtained and interpreted. The relationships among the abovementioned parameters and $\mathrm{a} / \mathrm{d}$ ratio, tensile reinforcement ratio and different concrete types were assessed, as well. Crack distributions and failure modes of beam specimens after testing are shown in Figure 6.6.

With the effects of increased loading during initial testing, bending cracks occurred in ECC beam with a/d ratios of 1.0 (ECC1_V_28D). Upon further increase in loading, the width of bending cracks increased and tensile reinforcements reached their yielding stress at $3.71 \mathrm{~mm}$ deflection with $403.6 \mathrm{kN}$ load. Failure occurred after exhibiting highly ductile behavior at maximum load of $501.47 \mathrm{kN}$ and mid-span deflection of $16.01 \mathrm{~mm}$. ECC beam with a/d ratio of 1.0 (ECC1_V_28D) failed in a typical flexural mode due to crushing at the top portion of the maximum moment region after yielding of the main longitudinal bottom steel reinforcement (Figure 6.6).

In ECC beam with a/d ratio of 2.0 (ECC2_V_28D, load increase initially caused the formation of bending cracks at the maximum moment region. Then shear cracks formed in the right shear span. The beam showed yielding of flexural reinforcement at $211.45 \mathrm{kN}$ loading with corresponding $12.58 \mathrm{~mm}$ deflection. The specimen failed after reaching maximum load carrying capacity of $220.66 \mathrm{kN}$. After the yielding of tensile reinforcements, flexural-shear failure took place due to excessive widening of shear cracks in the right shear span, and subsequent propagation of these cracks to the right loading point (Figure 6.6).

ECC beam with a/d ratio of 3.0 (ECC3_V_28D) showed flexural failure due to crushing of concrete at the maximum moment region after the yielding of tensile reinforcement and reaching its yield and maximum load carrying capacities of $121 \mathrm{kN}$ (at $11.79 \mathrm{~mm}$ deflection) and $143 \mathrm{kN}$, respectively. 

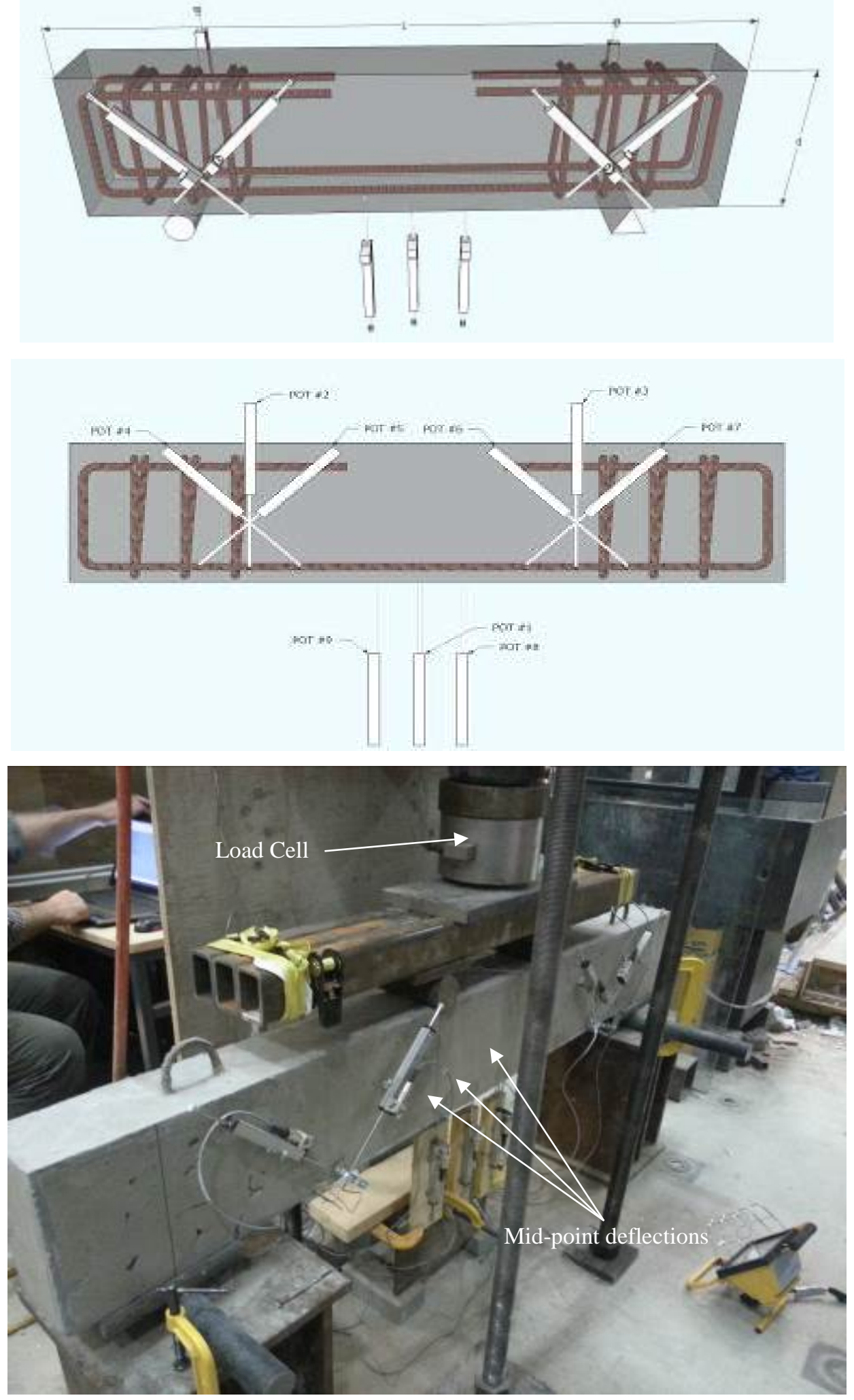

Figure 6.3: Test setup and instrumentation 
Although ECC beams were with no shear reinforcements, the beams resulted in flexural failure. The reason for that could be due to the nature of ECC mix design; thanks to the multiple cracking and fiber bridging behaviors. The beams failed under flexure after displaying ductility and energy absorption capacity.

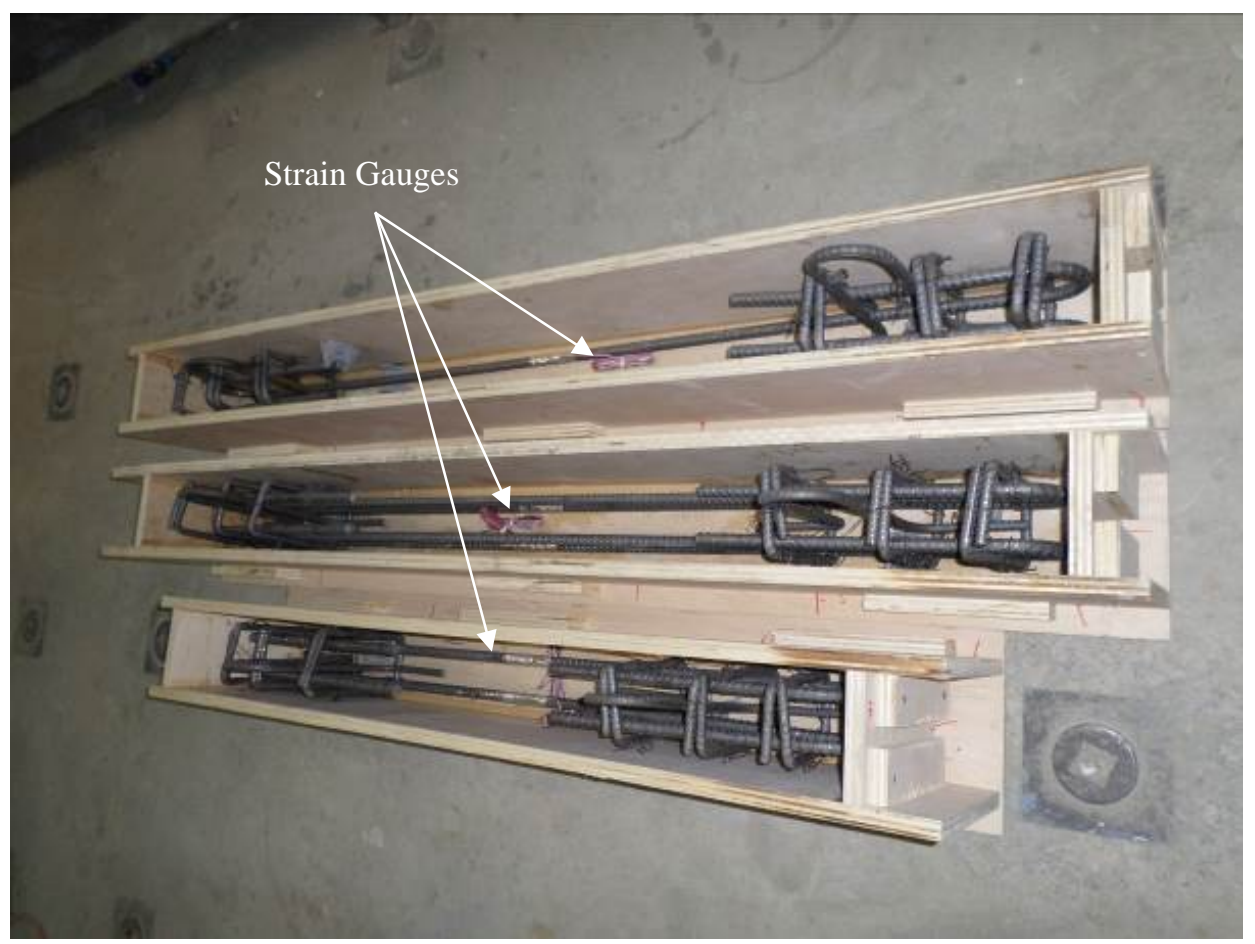

Figure 6.4: Strain measurements of longitudinal tensile reinforcements

\subsubsection{Strength and stiffness of beams and influence of self-healing}

Strength values of the beam specimens were determined for two levels and are presented in Table 6.2. These values, which correspond to the steel yielding and ultimate (peak) loads, were obtained from load-deflection curves (Figure 6.5). As expected, both yield and ultimate strengths of virgin ECC beams decreased with the increase of shear span to effective height ratio $(a / d)$. For virgin ECC beams, yield strength dropped by $91 \%$ when the a/d ratio was increased from 1 to 2 . When the a/d ratio was increased from 2 to 3, the reductions were less pronounced, $75 \%$ decrease was observed.

The stiffness values of the tested beams as presented in Table 6.2 were calculated at the point of steel yielding from load-deflection responses. In general, the stiffness of virgin ECC beam with a/d ratio of 1 (ECC1_V_28D) was higher than virgin ECC beams with a/d ratios of 2 and 3 (ECC2_V_28D and ECC3_V_28D). 


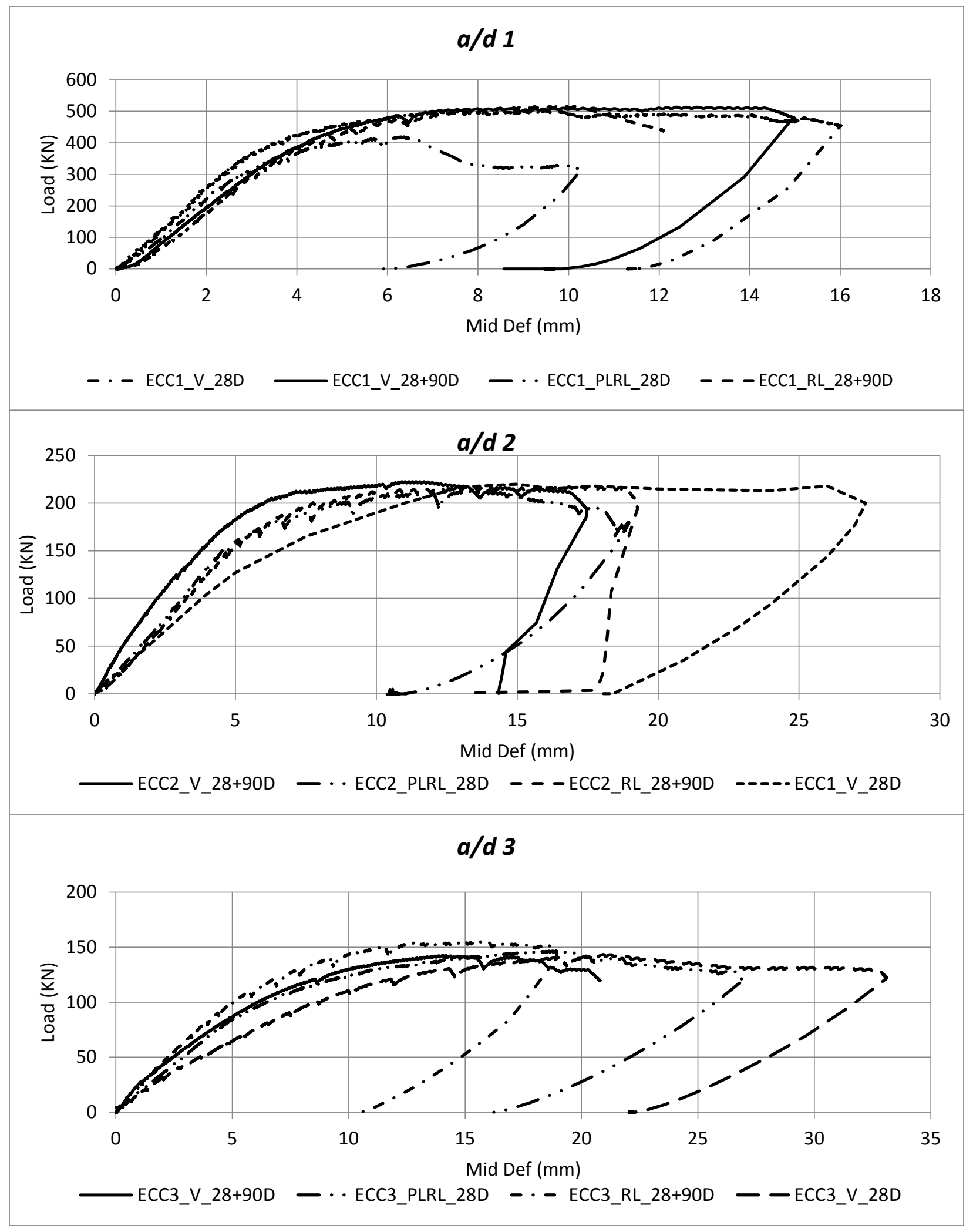

Figure 6.5: Load-deflection curves of specimens 

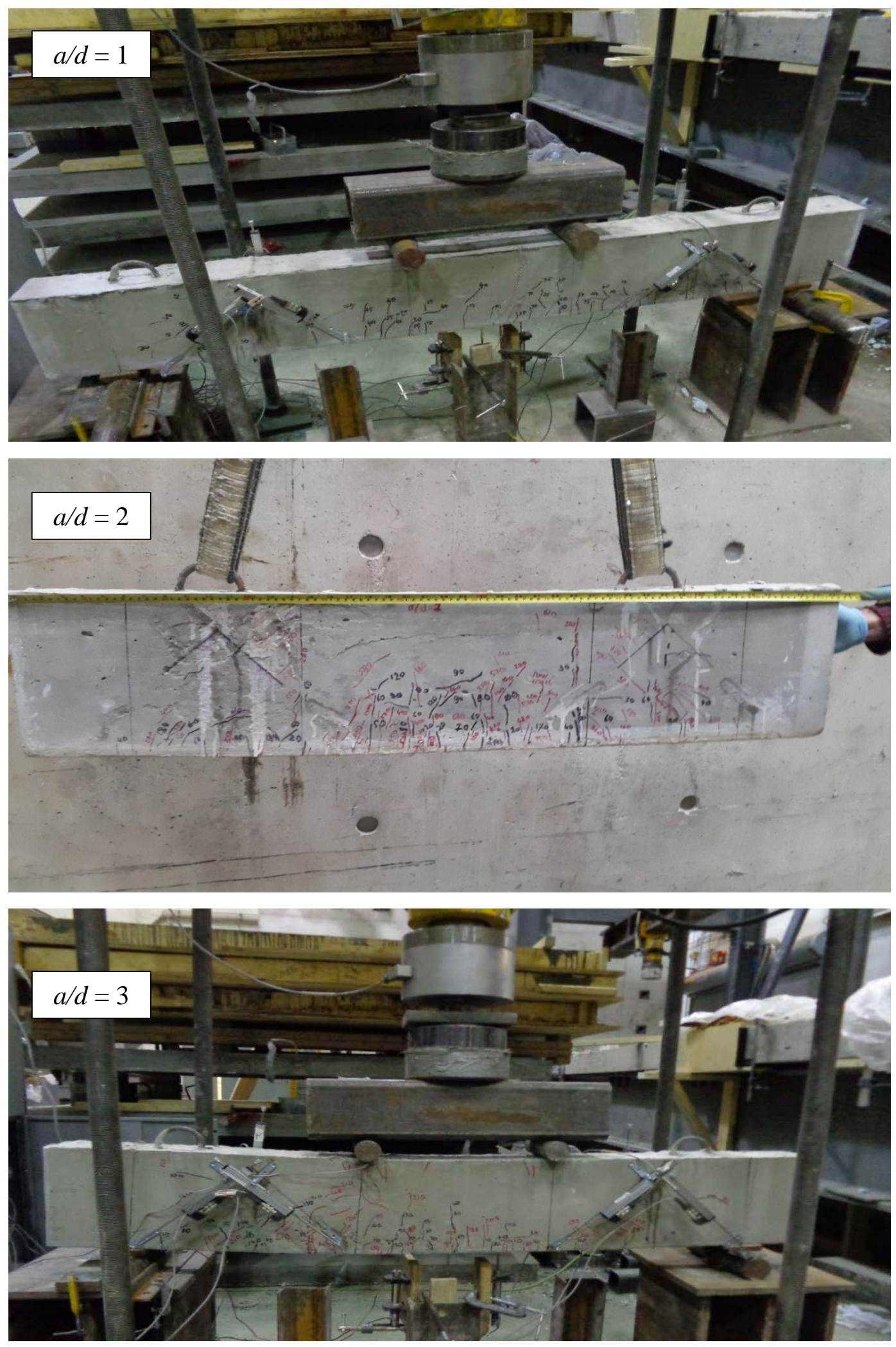

Figure 6.6: Crack distributions and failure modes of ECC specimens 
Table 6.2: Overall results of the beam specimens after four-point bending tests

\begin{tabular}{|c|c|c|c|c|c|c|c|c|c|}
\hline \multirow{2}{*}{$\begin{array}{l}\text { Spec } \\
\text { No. }\end{array}$} & \multirow{2}{*}{$\begin{array}{c}\text { Concrete } \\
\text { Type/Beam Name }\end{array}$} & \multicolumn{2}{|c|}{$\begin{array}{c}\text { Load Capacity } \\
(\mathbf{k N})\end{array}$} & \multicolumn{2}{|c|}{$\begin{array}{c}\text { Mid Span Deflection } \\
\text { (mm) }\end{array}$} & \multirow{2}{*}{$\begin{array}{c}\text { Ductility } \\
\text { Ratio }\end{array}$} & \multirow{2}{*}{$\begin{array}{c}\text { Yield Stiffness } \\
\text { (kN/mm) }\end{array}$} & \multirow{2}{*}{$\begin{array}{c}\text { Energy } \\
\text { Absorption } \\
\text { Capacity } \\
\text { (Joules) }\end{array}$} & \multirow{2}{*}{ Failure Mode } \\
\hline & & Yield & Ultimate & At Yield & At Failure & & & & \\
\hline 1 & ECC1_V_28D & 403.60 & 501.47 & 3.71 & 16.01 & 4.32 & 108.79 & 6113 & Flexure \\
\hline 2 & ECC1_PLRL_28D & 335.82 & 419.12 & 3.30 & 10.26 & 3.11 & 110.05 & 2673 & Flexure \\
\hline 3 & ECC1_RL_28+90D & 320.55 & 516.56 & 3.39 & 12.12 & 3.58 & 90.08 & 4560 & Flexure \\
\hline 4 & ECC1_V_28+90D & 432.32 & 513.81 & 4.77 & 14.99 & 3.15 & 117.06 & 5308 & Flexure \\
\hline 5 & ECC2_V_28D & 211.45 & 220.66 & 12.58 & 27.36 & 2.17 & 16.81 & 4269 & Flexure-Shear \\
\hline 6 & ECC2_PLRL_28D & 159.63 & 213.96 & 4.99 & 18.96 & 3.79 & 33.16 & 2643 & Flexure \\
\hline 7 & ECC2_RL_28+90D & 158.53 & 217.49 & 5.09 & 19.27 & 3.80 & 33.40 & 3138 & Flexure \\
\hline 8 & ECC2_V_28+90D & 150.24 & 222.72 & 4.21 & 17.46 & 4.14 & 39.17 & 2854 & Flexure \\
\hline 9 & ECC3_V_28D & 120.96 & 143.27 & 11.79 & 32.75 & 2.78 & 10.26 & 3093 & Flexure \\
\hline 10 & ECC3_PLRL_28D & 96.65 & 146.64 & 6.17 & 26.92 & 4.37 & 17.36 & 2529 & Flexure \\
\hline 11 & ECC3_RL_28+90D & 103.86 & 154.96 & 5.3 & 18.94 & 3.58 & 21.18 & 1776 & Flexure \\
\hline 12 & ECC3_V_28+90D & 100.65 & 142.53 & 6.14 & 20.79 & 3.39 & 16.75 & 2267 & Flexure \\
\hline
\end{tabular}


When focus is placed on the effect of pre-loading on the strength and stiffness results, it is found that the pre-loading up to $50 \%$ of the ultimate bending deformation resulted in the decrement of both yield and ultimate loads but in an increase of stiffness of ECC1_PLRL_28D (1.14\% increase), ECC2_PLRL_28D (49\% increase), ECC3_PLRL_28D (40.9\% increase) beams compared to their ECC1_V_28D, ECC2_V_28D and ECC3_V_28D counterparts (Table 6.2). These findings showed the effectiveness of pre-loading in lowering strength and increasing the stiffness results and that the $\mathrm{a} / \mathrm{d}$ ration was a decisive parameter in damage tolerance. The reason for this finding could be due to the nature of ECC mixture; the multiple cracking and damage tolerance behavior.

Considering the effect of self-healing on damaged specimens (subjected to pre-loading up to 50\% of their ultimate bending deformation capacity after 28 days and cured under water for further 90 days), one can see that recoveries resulting in strength values close or even slightly higher are achievable regardless of a/d ratio (Table 6.2). However, more healing was anticipated for beam specimens with a/d ratio of 1.0 and the reason for this finding could be due to the difference in the crack formation and crack width between the specimens with different a/d ratios. Specimens with a/d ratio of 1.0 showed more cracks with average crack width between $50-100 \mu \mathrm{m}$ and those cracks are more likely to express autogenous healing over the larger cracks in the case of a/d ratios of 2 and 3 (Figure 6.6). Also, the crack re-opening is dominant factor following the reloading of the beams after the curing period. If the same crack opened again after the reloading, then this will affect the regain of the properties. For example, ultimate load carrying capacities of ECC1_PLRL_28D, ECC2_PLRL_28D and ECC3_PLRL_28D beams (419.12 kN, 213.96 kN and $146.64 \mathrm{kN}$, respectively) were surpassed by ECC1_RL_28+90D, ECC2_RL_28+90D and ECC3_RL_28+90D beams (516.56 kN, 217.49kN and 154.96 kN, respectively (Figure 6.7).

To support this finding, a comparison was made between large scale beams and small prism specimens (discussed in chapter 5). Similar procedure was used to evaluate the healing in both large and small sale specimens. The prisms were subjected to pre-loading up to $75 \%$ of their midspan beam deflection values and kept without sustained loading for curing. One can see that recoveries resulting in values close or even slightly higher than that of those sound specimens. For example, while MOR results of sound ECC_L prism specimens was 11.1 MPa curing, initial preloading and subsequent water exposure resulted in final values of 11.2 MPa. These results show that after water curing, the ECC_L prism and large scale specimens attained average results greater 
than or close to the sound specimens. Additionally, these findings offer an understanding into the nature of final self-healing products, which were also visually monitored on the crack faces of different specimens (Figure 6.5 and Figure 6.6). This suggest that specimens with fly ash (regardless of their size) further-formed C-S-H gels and it was the dominant self-healing products.

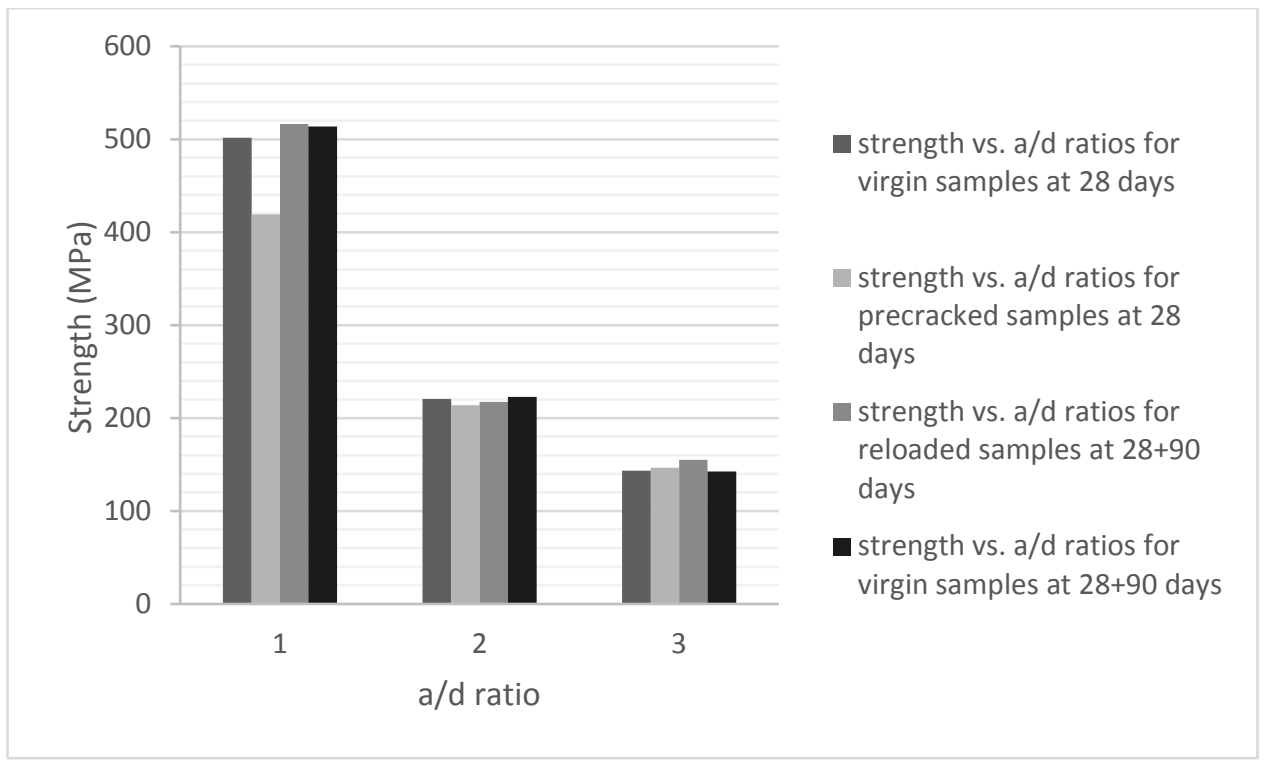

Figure 6.7: Strength vs a/d ratios

In general, reloaded healed ECC beams showed lower yield load compared to their virgin counterparts. For example, yield load as \% of ultimate load for ECC1_RL_28+90D (pre-cracked and left to heal for additional 90 days) beam was $62 \%$ compared to $84 \%$ of its virgin counterpart ECC1_V_28+90D. The rebar yielding at lower load indicated that healed pre-cracked/damaged beams did not recover fully (through self-healing) which allowed ECC matrix to contribute less to the overall load resistance due to early cracking and subsequent transfer of load to the reinforcing steel causing rebar to yield at lower load. Although ECC beams exhibited self-healing characteristics during 90 days of curing period, long-term study should be conducted to reveal fully the self-healing potential.

\subsubsection{Deflection ductility and self-healing}

The deflection ductility was defined as the ratio of deflection at $85 \%$ of post-peak/ultimate load (considered as failure point) to that at steel yielding. The calculated ductility ratios of the beam specimens are shown in Table 6.2. Based on the deflection ductility ratio, it can be concluded that higher ductility ratio was acquired by virgin ECC beam with a/d ratio of 1.0 (ECC1_V_28D). In 
general, deflection ductility ratios show a decreasing trend with the increase of a/d ratio with the exception of virgin ECC beam with a/d of 2.0 (ECC2_V_28D). The ductility of virgin ECC beam with a/d ratio of 1.0 (ECC1_V_28D) was 49\% and 35\% higher than the ductility of ECC beams with a/d ratio of 2.0 and 3.0 (ECC1_V_28D and ECC2_V_28D), respectively (Figure 6.8).

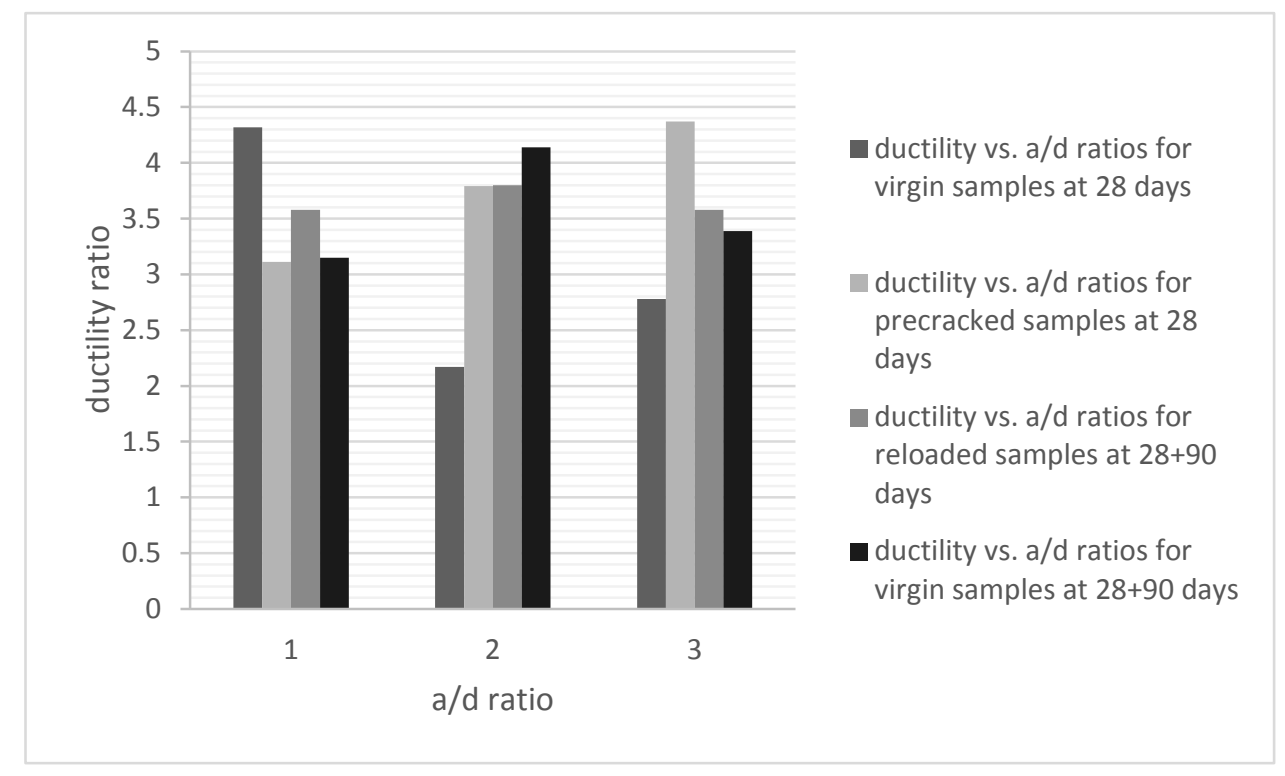

Figure 6.8: Ductility ratio vs a/d ratios

Initial pre-loading of the specimens before sudden re-loading significantly reduced the deflection evolution. For example, the average ultimate deflection of 28-day-old virgin ECC1_V_28D specimen was reduced from $16.01 \mathrm{~mm}$ to $10.26 \mathrm{~mm}$ due to pre-loading. Moreover, decrement rates in deflection were more than the decrements in strength values. For instance, the rate of decrease in strength values of ECC1_V_28D specimens was 16.42\% after pre-loading compared to 35.9\% of deflection. This might be attributed to faster localization of already introduced individual microcracks, which cause final failure without significantly sacrificing maximum flexural load upon re-loading (Figure 6.8).

Comparison between beams (subjected to pre-loading at the age of 28 days then released and reloaded up to failure and those subjected to pre-loading and left to self-heal for 90 days under water before loaded to failure) exhibited ductility recoveries for a/d ratio of 1.0 (ductility ratio ranged from 3.10 to 3.58). This situation did not hold true for beams with a/d ratio of 3 - the recovery in the deflection ductility ratio was not visible. For example, the deflection ductility ratio of ECC3_PLRL_28D beam was 4.37 to 3.58 for ECC3_RL_28+90D beam. Similar behavior (like 
pre-loaded specimens) was exhibited by those virgin specimens directly tested up to failure after 118 days (28 days + 90 days) of initial curing. For example, deflection ductility ratios of 118-dayold virgin ECC1_V_90D, ECC2_V_90D and ECC3_V_90D beams were 3.15, 4.14 and 3.39, respectively. For ECC1_PLRL_28D, ECC2_RL_28+90D and ECC3_RL_28+90D beams kept in water for 90 days after pre-loading, the ductility ratios were 3.58, 3.79 and 3.58, respectively (Figure 6.8).

\subsubsection{Energy absorption capacity and recovery due to self-healing}

The energy absorption capacity of beams was calculated as the area under load-deflection curves - only the area up to the failure point defined in ductility ratio calculations explained before. Energy absorption capacities of all the beams are presented in Table 6.2. Energy absorption capacity of the beams decreased dramatically with the increase of a/d ratio. For a/d ratios of 1, 2 and 3, energy absorption capacities of the virgin ECC beams (ECC1_V_28D, ECC2_V_28D and ECC3_V_28D) were 6113, 4269 and 3093 Joules, respectively (Figure 6.9).

The application of initial pre-loading decreased the energy absorption capacity of ECC beams regardless of a/d ratio. For example, the energy absorption capacity of 28-day-old virgin ECC2_V_28D beam reduced from 4269 Joules to 2643 Joules due to applied pre-loading; this situation held true for rest of beams. Moreover, decrement rates in the energy absorption results were higher in the case of a/d ration of 1 . For instance, the rate of decrease in the energy absorption values of ECC1_V_28D specimens was 35.9\% after pre-loading. The lowest decrement was noticed in the case of a/d ratio of 3 (ECC3_V_28D) (Figure 6.9).

As the curing period was extended, improvement in the energy absorption capacity results was noted due to the continuous evolution of cementitious composites with time (especially for beams with a/d ratio of 1 and 2). This situation did not hold true for beams with a/d ratio of 3 . For example, the energy absorption capacity of ECC3_PLRL_28D beam was 2529 Joules and it reduced to 1776 for ECC3_RL_28+90D beams - which signified that no improvement in energy absorption capacity due to self-healing. Same behavior was exhibited by those of virgin specimens directly tested up to failure after 118 days (28 days +90 days) of initial curing. For example, the energy absorption capacities of 118-day-old virgin ECC1_V_90D, ECC2_V_90D and ECC3_V_90D beams were 5308, 2854 and 2267 Joules, respectively compared to 4560, 3138 and 1776 Joules, 
respectively of ECC1_PLRL_28D, ECC2_RL_28+90D and ECC3_RL_28+90D beams in water for 90 days after pre-loading - no energy recovery due to self-healing (Figure 6.9). The reason for this behavior could be due to the nature of the crack formation and crack width as was explained above in the strength and stiffness section.

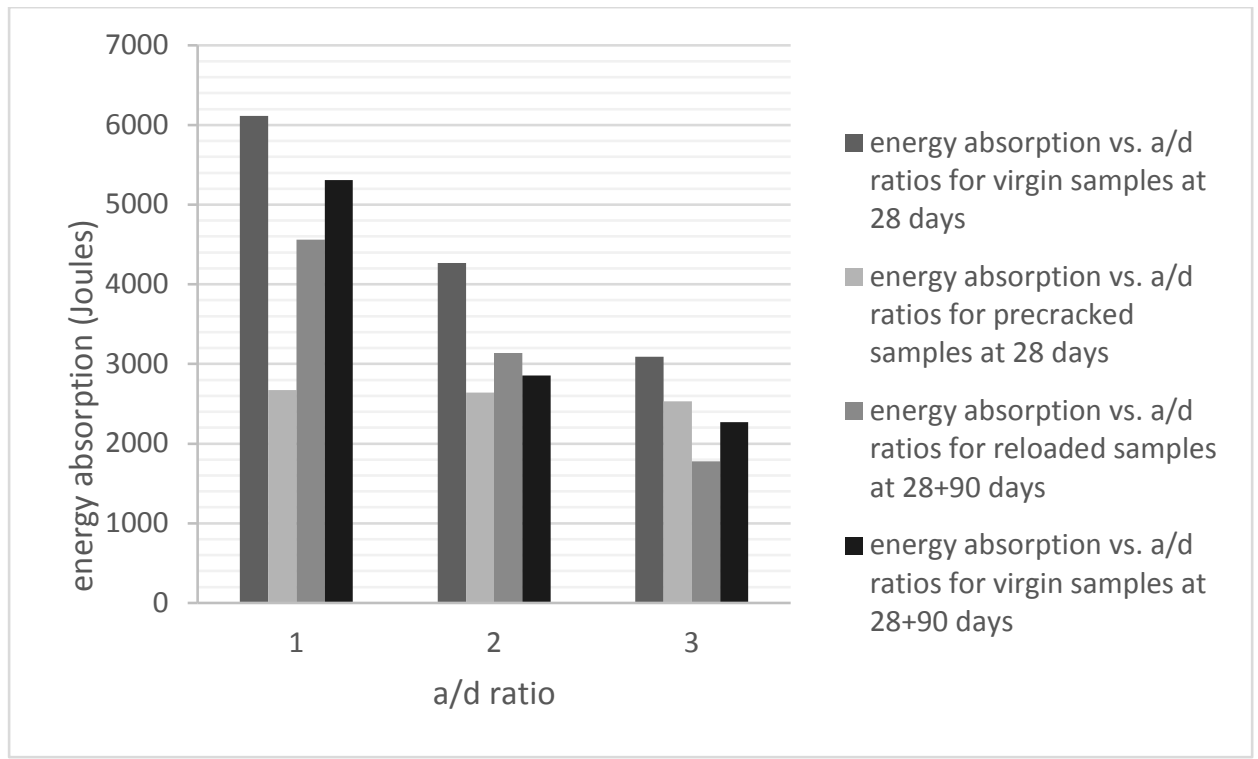

Figure 6.9: Energy absorption vs a/d ratios

\subsection{Chapter summary}

In this chapter, the self-healing behavior of reinforced ECC beams under four-point bending loading (with shear span to effective height 'a/d' ratio as the main variable parameter) was described. Initially 28 day cured twelve beams (virgin + loaded to failure-28day, preloaded+reloaded to failure-28day, 28day cured+preloaded+90-day water cured + reloaded to failure and virgin + cured in water 118day + loaded to failure) were tested under varying loading and curing schemes to study the self-behavior based on the recovery of strength, stiffness, ductility and energy absorbing capacity. The relationships among a/d ratio, load carrying capacity, ductility ratio, energy absorption capacity and stiffness of virgin and healed beams as well as failure modes and load-deflection responses of beam were used to quantify the self-healing of the ECC beams. 


\section{Chapter 7}

\section{Conclusions and recommendations for future studies}

\subsection{Introduction}

This research focused on, the durability of microcracked ECCs incorporating different mineral admixtures with varying chemical compositions and, the effects of progressively increasing sustained loading on ECC samples. Moreover, the self-healing capability of ECC was investigated based on structural performance of reinforced ECC beams.

\subsection{Conclusions}

Through this research, the robustness of self-healing was quantified and the following conclusions were obtained:

1. Chapter 3, entitled "Effects of Microcracking and Type of Cementitious Materials on the Durability Performance of Cementitious Composites” presented the relationship among the microcracking and chloride ion permeability of concrete mixtures that contain different supplementary cementitious materials along with the effects of different SCM types on some of the mechanical properties. The following conclusions were drawn from the results:

- Compressive strength values of ECC_S specimens were found the highest at the end of each specified curing age. However, even the lowest compressive strength value obtained from mixtures was more than needed for many civil engineering applications.

- Flexural strength results showed variation between the values of 7.5 and $11.9 \mathrm{MPa}$ based on the changes in initial curing ages and SCM types. As in compressive strength results the highest flexural strength values were obtained in the case of ECC_S specimens and there was an increase in the results with time although the extent was less pronounced compared to compressive strength results.

- Flexural deformation values of ECC specimens were in between the values of 2.2 and $5.6 \mathrm{~mm}$ depending on age and mixture type. Lowest flexural deformation capacity was obtained from ECC_S specimens at the end of each age which was related with the improved matrix maturity. However, the lowest flexural deformation capacity obtained 
from ECC_S specimens was nearly 200 times that of normal concrete and conventional fiber reinforced concrete.

- As opposed to water permeability, cracking significantly affected the chloride ion penetrability of ECC specimens. Despite the increments in RCPT results with the application of pre-loading however all of the final values at the end 90 days were in either in low or in moderate level as stated by ASTM C 120.

- Air-void characteristics appear to not be the sole factor influencing the frost durability of different ECC mixtures in terms of sorptivity measurements. Although some parameters (i.e. specific surface and spacing factor) do not fall into the required limits for adequate freeze-thaw durability, results from both sound and pre-loaded specimens are not alarming even after $300 \mathrm{~F} / \mathrm{T}$ cycles.

- Application of pre-loading caused exponential increments in water sorptivity results of different ECCs, regardless of the number of cracks introduced. Although increased cracks numbers were accompanied by increased sorptivity results for all mixtures, there were more sudden escalations in the sorptivity results of ECC_S specimens when more cracks were introduced, which is correlated with the effects of slag utilization on different crack characteristics. Despite the higher increments in sorptivity results with the increased crack numbers, however, the lowest values were acquired from the sound ECC_S mixture specimens, even after severe microcracking.

- All ECC mixtures incorporating different fly ash types showed marked reductions in the sorptivity results of both sound and microcracked specimens, up to the end of 150 $\mathrm{F} / \mathrm{T}$ cycles in some cases. Beyond $150 \mathrm{~F} / \mathrm{T}$ cycles, however, results started to increase for both sound and pre-loaded specimens, although increments were more visible for microcracked specimens. On the other hand, no reduction was monitored for ECC_S specimens at any stage of the F/T cycles. This opposition between the fly ash and slag bearing ECCs was found to be attributable to the differences in the formation processes of different self-healing products for pre-loaded specimens, as well as differences in further binding capability in sound specimens.

- Although sorptivity measurements of ECCs substantially varied depending on crack characteristics, number of $\mathrm{F} / \mathrm{T}$ cycles and so on, even the highest sorptivity value obtained after $300 \mathrm{~F} / \mathrm{T}$ cycles was restricted to $0.1490 \mathrm{~mm} / \mathrm{min}^{1 / 2}$, which is, according 
to the literature, equivalent to almost half of the value obtained from sound conventional concrete.

2. Chapter 4, "Effect of Self-Healing on the Different Transport Properties of Cementitious Composites incorporating different mineral admixtures”, focused on the effects of selfhealing on the different transport properties of microcracked Engineered Cementitious Composites with different maturity levels and incorporating different mineral admixtures. The following conclusions from chapter 4 were drawn:

- The influence of pre-cracking on sorptivity results of different ECC mixtures showed exponential increments in values when microcracks were introduced into the specimens. The effect of microcracking on sorptivity results appeared to increase its intensity when specimens were cured for longer periods.

- Despite the rapid escalations in results with the application of pre-loading, $92 \%$ of healing in the sorptivity measurements was shown to be possible depending on the type of mineral admixture used in the composites, the duration of the initial aging (maturity level) and further CW curing. Contrary to expectations, ECC_S specimens were influential in lowering sorptivity results through self-healing even more than fly-ashbearing ECCs in some cases.

- Chloride ion penetrability results of ECC mixtures increased markedly in the presence of microcracks, although the extent was highly dependent on maturity level and type of mineral admixture used in the mixtures.

- When additional CW curing was applied to the specimens, self-healing realized through improvements in chloride ion penetrability attracted great attention; the RCPT results of most of the almost-failed ECC specimens decreased to low levels according to ASTM C 1202 at 30 days of CW curing.

- When a comparison is made between the healing rates obtained from sorptivity and RCPT measurements, sorptivity results showed sudden drops after only seven days of CW curing, while this period was longer (30 days) for RCPT results. The reason for the differences in self-healing performance in the different tests is believed to be due to significantly varying parameters, which have major effects on the individual tests.

3. Chapter 5, entitled “Assessing the Self-Healing Capability of Cementitious Composites under Progressively Increasing Mechanical Loading”, investigated the effects of 
progressively increasing sustained loading on self-healing behavior of 180-day-old microcracked ECC incorporating different mineral admixtures. The following conclusions have been drawn based on the results:

- 150 days of continuous moist curing led to MOR results of initially pre-loaded, 180day-old ECC specimens not subjected to progressive sustained loading to be close and/or higher than those of sound specimens, depending on the type of MA used in the mixtures. This finding was informative regarding the strength of final self-healing products stemming from different ECCs with varying MA utilization.

- Application of sustained loading, intensified monthly beyond severe initial pre-loading, did not cause significant changes in MOR results when specimens were subjected to moist curing for 150 days. Results similar to those of sound specimens were achievable, although recovery rates were slightly lower than in specimens with no sustained loading.

- Deflection results were very close for specimens failed after 180 days of initial curing and those initially pre-loaded on the $180^{\text {th }}$ day and exposed to 150 days of moist curing without any sustained loading. When a similar comparison was made with specimens subjected to progressive sustained loading, there were reductions in the recovery results. However, even the lowest deflection values acquired under progressive sustained loading (1.6 mm in ECC_S) were still more than hundred times those of conventional concrete.

- Initial pre-loading and subsequent exacerbation with sustained loading after 180 days did not make a significant difference in the original UPV results. This is believed to be due to the lower precision of UPV testing in capturing related data.

- Self-healing performance results in terms of UPV measurements were close to each other in specimens subjected only to initial pre-loading before moist curing, and in those subjected to progressively increasing sustained loading after initial pre-loading. Although UPV recovery rates were not that marked, 30 days of continuous moist curing was more than enough for all ECCs to achieve UPV results higher than those of the sound specimens.

4. Chapter 6, "Self-healing behavior of full-scale Cementitious Composites with different shear span to depth ratio", investigated the self-healing behavior of large scale ECC 
reinforced beams under load influence by giving priority to the individual effects of ultrahigh ductility with moderate strength of ECC beams. This study has reached the following conclusions:

- The use of ECC in beam specimens without shear reinforcement successfully prevented the shear failure and resulted in flexural failure due to the damage tolerance of ECC mix. Tensile reinforcements of all reinforced beams with a/d ratio ranging from 1 to 3 (expected to fail in shear) reached their yielding capacity. Both yield and ultimate strengths of ECC beams decreased with the increase of shear span to effective height ratio. ECC beams showed ductile failure with displacement ductility of more than 2.0.

- ECC beams with no regards to their a/d showed strength recovery after 90 days of extended moisture curing after pre-cracking. However, ECC beams with $a / d$ of 1.0 exhibited the highest recovery compared to the those pre-loaded up to $50 \%$ of their ultimate load then re-loaded up to failure at the age of 28 days.

- Based on the deflection ductility ratio, ECC beams with a/d of 1.0 and 2.0 showed recovery in the ductility and it was more pronounced beams with a/d of 1.0. On the contrary, no ductility recovery was noticed for beams with $a / d$ of 3 . The finding could be related to the crack width and the possible reopening of the healed cracks which affected the recovery of the properties.

- ECC beams with $a / d$ of 1.0 and 2.0 displayed better recovery in the energy absorption capacity than those having a/d of 3.0. Energy absorption capacities and the recovery decreased with the increase of a/d.

- Experimental findings showed that ECC beams with a/d ratio of 1 showed the highest recovery in the results in different parameters that were considered in this chapter. The recovery in the values reduced as the $a / d$ ratios increased in general. Although, beams with a/d ratio of 3 showed less or no recovery, the partial healing that happened in this $a / d$ ratio beam is still considered acceptable in most civil engineering applications.

\subsection{Future work}

The research presented in this thesis provides a preliminary database on the quantification of selfhealing capability ECC mixtures incorporating pozzolanic mineral admixtures showing promising 
results. Indeed, it may be of interest to investigate new aspects related to the achievement of robust self-healing by considering the following points as future research:

- Continue the research on self-healing using other types of supplementary cementitious materials such as nano-silica and glass powder.

- Expand the work on self-healing behavior of full-scale ECC beams/frames) using other supplementary materials and different curing regimes.

- Study the life-cycle cost analysis for self-healing ECC using intrinsic healing with selfcontrolled tight crack width as a base to compare with other available self-healing techniques.

- Conduct research on self-healing under field exposures (such as highly variable temperature and precipitation) which may validate self-healing methods/techniques that look promising under highly controlled conditions in the laboratory. 


\section{References}

ACI Committee 116R. (2005). Cement and Concrete Terminology. American Concrete Institute. Farmington Hills, Michigan.

ACI Committee 318. (2014). Building Code Requirements for Structural Concrete and Commentary. American Concrete Institute. Farmington Hills, Michigan.

ACI Committee 345. (2011). Guide for concrete highway bridge deck construction. American Concrete Institute. Farmington Hills, Michigan.

ASTM Standard C39/C39M-16. (2016). Standard Test Method for Compressive Strength of Cylindrical Concrete Specimens. ASTM International. West Conshohocken, PA.

ASTM Standard C120/C120M-15a. (2015). Standard Test Methods for Flexure Testing of Structural and Roofing Slate. ASTM International. West Conshohocken, PA.

ASTM Standard C231/C231M-14, (2014). Standard Test Method for Air Content of Freshly Mixed Concrete by the Pressure Method. ASTM International. West Conshohocken, PA.

ASTM Standard C457/C457M-12, (2012). Standard Test Method for Microscopical Determination of Parameters of the Air-Void System in Hardened Concrete. ASTM International. West Conshohocken, PA.

ASTM Standard C597-16. (2016). Standard Test Method for Pulse Velocity Through Concrete. ASTM International. West Conshohocken, PA.

ASTM Standard C618-15, (2015). Standard Specification for Coal Fly Ash and Raw or Calcined Natural Pozzolan for Use in Concrete. ASTM International. West Conshohocken, PA.

ASTM Standard C666/C666M-15, (2015). Standard Test Method for Resistance of Concrete to Rapid Freezing and Thawing. ASTM International. West Conshohocken, PA.

ASTM Standard C1202-12, (2012). Standard Test Method for Electrical Indication of Concrete's Ability to Resist Chloride Ion Penetration. ASTM International. West Conshohocken, PA. 
ASTM Standard C1585-13, (2013). Standard Test Method for Measurement of Rate of Absorption of Water by Hydraulic-Cement Concretes, ASTM International. West Conshohocken, PA.

Aldea, C., Song, W., Popovics, J. and Shah, S. (2000). Extent of Healing of Cracked Normal Strength Concrete. J. Mater. Civ. Eng., 12(1), pp.92-96.

Alexander, M., Jaufeerally, H. and Mackechnie, J. (2003). Structural and Durability Properties of Concrete Made by Corex Slag. Research Monograph No. 6. South Africa: Departments of Civil Engineering University of Cape Town.

Alexander, M. and Stanish, K. (2005). Durability design and specification of reinforced concrete structures using a multi-factored approach. In: ConMat'05.

Anstice, D. J., Page, C. L., \& Page, M. M. (2005). The pore solution phase of carbonated cement pastes. Cement and Concrete Research, 35(2), 377-383.

Bakker, R. (1988). Initiation Period of Corrosion. RILEM Report: Corrosion of Steel in Concrete. pp.22-55.

Birchall, J., Howard, A. and Kendall, K. (1981). Flexural strength and porosity of cements. Nature, 289(5796), pp.388-390.

Bentz, D. (2006). Influence of alkalis on porosity percolation in hydrating cement pastes. Cement and Concrete Composites, 28(5), pp.427-431.

Bleedens, A. and Vandewalle, L. (2001). Durability of high strength concrete for highway pavement restoration. In: CONSEC '01: Third International Conference on Concrete Under Severe Conditions. pp.1230-1238.

Ciampoli, M. (2001). Aging Effects, Maintenance, and Structural Health Monitoring. In: Structural Safety and Reliability. Swets \& Zeitlinger.

Chang, P., Peng, Y. and Hwang, C. (2001). A design consideration for durability of highperformance concrete. Cement and Concrete Composites, 23(4-5), pp.375-380. 
Chindaprasirt, P., Jaturapitakkul, C. and Sinsiri, T. (2005). Effect of fly ash fineness on compressive strength and pore size of blended cement paste. Cement and Concrete Composites, 27(4), pp.425428.

Drabik, M. and Slade, R. (2004). Macrodefect-Free Materials: Modification of Interfaces in Cement Composites by Polymer Grafting. Interface Science, 12(4), pp.375-379.

Edvardsen, C. (1999). Water Permeability and Autogenous healing of cracks in concrete, ACI Materials Journal, 96(4), pp.48-455.

Fan S., Li M. (2015). Computed Microtomography of Three-Dimensional Microcracks and SelfHealing in Engineered Cementitious Composites. Smart Mater. Struct., 24(1):015021.

Farage, M., Sercombe, J. and Gallé, C. (2003). Rehydration and microstructure of cement paste after heating at temperatures up to $300^{\circ} \mathrm{C}$. Cement and Concrete Research, 33(7), pp.1047-1056.

Glasser, F. (1991). Chemical, Mineralogical, and Microstructural Changes Occuring in Hydrated Slag-Cement Blends. Materials Science of Concrete II, pp.41-81.

Gerard, B., Reinhardt, H. and Breysse, D. (1997). Measured transport in cracked concrete. Penetration and Permeability of Concrete: RILEM Report 16. pp.265-331.

Hannant, D. and Keer, J. (1983). Autogenous healing of thin cement based sheets. Cement and Concrete Research, 13(3), pp.357-365.

Hassan, A., Lachemi, M. and Hossain, K. (2012). Effect of metakaolin and silica fume on the durability of self-consolidating concrete. Cement and Concrete Composites, 34(6), pp.801-807.

Herbert, E. and Li, V. (2001). Self-healing of engineered cementitious composites in the natural environment. In: High Performance Fiber Reinforced Cement Composites.

Hossain, K. (2003). Blended cement using volcanic ash and pumice. Cement and Concrete Research, 33(10), pp.1601-1605.

Hossain, K. (2004). Properties of volcanic pumice based cement and lightweight concrete. Cement and Concrete Research, 34(2), pp.283-291. 
Hossain, K. (2005). Volcanic ash and pumice as cement additives: pozzolanic, alkali-silica reaction and autoclave expansion characteristics. Cement and Concrete Research, 35(6), pp.11411144.

Engineers Canada, (2008). Adapting to climate change: Canada's first national engineering vulnerability assessment of public infrastructure.

Hearn, N. (1999). Effect of shrinkage and load-induced cracking on water Permeability of concrete, ACI Materials Journal, 96(2), pp.234-241.

Hwang, C., Liu, J., Lee, L. and Lin, F. (1996). Densified Mixture Design Algorithm and Early Properties of High Performance Concrete. Journal of the Chinese Institute of Civil and Hydraulic Engineering, 8(2), pp.217-229.

Ismail, M., Toumi, A., François, R. and Gagné, R. (2004). Effect of crack opening on the local diffusion of chloride in inert materials, Cement and Concrete Research, 34(4), pp.711-716.

Jacobsen, S., Marchand, J. and Hornain, H. (1995). SEM observations of the microstructure of frost deteriorated and self-healed concretes, Cement and Concrete Research, 25(8), pp.1781-1790.

Jacobsen, S., Marchand, J. and Boisvert, L. (1996). Effect of cracking and healing on chloride transport in OPC concrete. Cement and Concrete Research, 26(6), pp.869-881.

Jawed, I. and Skalny, J. (1978). Alkalis in cement: A review II. Effects of alkalis on hydration and performance of Portland cement. Cement and Concrete Research, 8, pp.37-52.

Jensen, O. and Hansen, P. (2002). Water-entrained cement-based materials. Cement and Concrete Research, 32(6), pp.973-978.

Jooss, M. (2001). Leaching of concrete under thermal influence. Otto-Graf-Journal, 12, pp.51-68.

Juenger, M. and Jennings, H. (2001). Effects of high Alkalinity on cement pastes, ACI Materials Journal, 98(3), pp.251-255.

Kan L.L., Shi H.S., Sakulich A.R., Li V. C. (2010). Self-Healing Characterization of Engineered Cementitious Composite Materials. ACI Materials Journal, 107(6): 617-624. 
Kanda, T. and Li, V. (1999). New Micromechanics Design Theory for Pseudostrain Hardening Cementitious Composite. J. Eng. Mech., 125(4), pp.373-381.

Karahan, O., Hossain, K., Ozbay, E., Lachemi, M. and Sancak, E. (2012). Effect of metakaolin content on the properties self-consolidating lightweight concrete. Construction and Building Materials, 31, pp.320-325.

Kim, J., Kim, J., Ha, G. and Kim, Y. (2007). Tensile and fiber dispersion performance of ECC (engineered cementitious composites) produced with ground granulated blast furnace slag. Cement and Concrete Research, 37(7), pp.1096-1105.

Kishi, T., Ahn, T., Morita, M. and Koide, T. (2011). Field test of self-healing concrete on therecovery of water tightness to leakage through cracks. In: 3rd International Conference on SelfHealing Materials.

Kosmatka, S. and Panarese, W. (1988). Design and control of concrete mixtures. 13th ed. Skokie, IL: Portland Cement Association, p.205.

Lauer, K. R. (1956, June). Autogenous healing of cement paste. In Journal Proceedings (Vol. 52, No. 6, pp. 1083-1098).

Lepech, M. and Li, V. (2005). Water permeability of cracked cementitious composites. In: Proceedings of 11th International Conference on Fracture. pp.20-25.

Lepech, M. and Li, V. (2009). Water permeability of engineered cementitious composites. Cement and Concrete Composites, 31(10), pp.744-753.

Leung, C. and Li, V. (1992). Effect of fiber inclination on crack bridging stress in brittle fiber reinforced brittle matrix composites. Journal of the Mechanics and Physics of Solids, 40(6), pp.1333-1362.

Leung, C. and Li, V. (1991). New strength-based model for the debonding of discontinuous fibres in an elastic matrix. J Mater Sci, 26(22), pp.5996-6010. 
Li, G. and Zhao, X. (2003). Properties of concrete incorporating fly ash and ground granulated blast-furnace slag. Cement and Concrete Composites, 25(3), pp.293-299.

Li, M., Sahmaran, M. and Li, V. (2007). Effect of cracking and healing on durability of engineered cementitious composites under marine environment. In: High Performance Fiber Reinforced Cement Composites (HPFRCC-5). pp.313-322.

Li, V. (1993). From Micromechanics to Structural Engineering - The Design of Cementitious Composites for Civil Engineering Application. Journal of Structural Mechanics and Earthquake Engineering, 10(2), pp.37-48.

Li, V. (1998). ECC - tailored composites through micromechanical modeling. In: Canadian Society for Civil Engineers Annual Conference. pp.64-97.

Li, V. (2003). On Engineered Cementitious Composites (ECC). Journal of Advanced Concrete Technology, 1(3), pp.215-230.

Li, V. (2012). Tailoring ECC for special attributes: A review. International Journal of Concrete Structures and Materials, 6(3). pp.135-44.

Li, V. and Herbert, E. (2012). Robust Self-Healing Concrete for Sustainable Infrastructure. Journal of Advanced Concrete Technology, 10(6), pp.207-218.

Li, V. and Stang, H. (1997). Interface property characterization and strengthening mechanisms in fiber reinforced cement based composites. Advanced Cement Based Materials, 6(1), pp.1-20.

Li, V., Wang, Y. and Backer, S. (1990). Effect of inclining angle, bundling and surface treatment on synthetic fibre pull-out from a cement matrix. Composites, 21(2), pp.132-140.

Li, V., Wang, Y. and Backer, S. (1991). A micromechanical model of tension-softening and bridging toughening of short random fiber reinforced brittle matrix composites. Journal of the Mechanics and Physics of Solids, 39(5), pp.607-625. 
Li, V., Wang, S. and Wu, C. (2001). Tensile strain-hardening behavior of polyvinyl alcoholengineered Cementitious composite (PVA-ECC), ACI Materials Journal, 98(6), pp.483492.

Li, V., Wu, C., Wang, S., Ogawa, A. and Saito, T. (2002). Interface tailoring for strain-hardening polyvinyl alcohol-engineered Cementitious composite (PVA-ECC), ACI Materials Journal, 99(5), pp.463-472.

Lin, Z., Kanda, T. and Li, V. (1999). On Interface Property Characterization and Performance of Fiber Reinforced Cementitious Composites. Journal of Concrete Science and Engineering, 1, pp.173-184.

Lin, Z. and Li, V. (1997). Crack bridging in fiber reinforced cementitious composites with sliphardening interfaces. Journal of the Mechanics and Physics of Solids, 45(5), pp.763-787.

Malhotra, V. (1998). Role of supplementary cementing materials in reducing greenhouse gasemission. CANMET Report MTL 98-03.

Mangat, P. and Gurusamy, K. (1987). Permissible crack widths in steel fibre reinforced marineconcrete. Materials and Structures, 20(5), pp.338-347.

Martys, N. and Ferraris, C. (1997). Capillary transport in mortars and concrete. Cement and Concrete Research, 27(5), pp.747-760.

Marlowe, I. (2003). Emission factors programme Task 4(b): Review of cement sector pollutioninventory.

Mather, B. and Warner, J. (2003). Why do concrete repairs fail? [online] Available at: http://aec.engr.wisc.edu/resources/rsrc07.html [Accessed 27 Feb. 2013].

Mehta, P. (1986). Concrete. Englewood Cliffs, N.J.: Prentice-Hall.

Mehta, P. (1998). Role of pozzolanic \& cementitious by-products in sustainable development of the concrete industry. In: 6th CANMET/ACI/JCI Conference: Fly Ash, Silica Fume, Slag \& Natural Pozzolans in Concrete. 
Mehta, P. and Burrows, R. (2001). Building durable structures in the 21st century. ConcreteInternational Journal, 23(3), pp.57-63.

Mihashi, H. and Leite, J. (2004). State-of-the-Art Report on Control of Cracking in Early Age Concrete. Journal of Advanced Concrete Technology, 2(2), pp.141-154.

Mindess, S. and Young, J. (2002). Concrete. Englewood Cliffs, N.J.: Prentice-Hall.

Misra, S., Yamamoto, A., Tsutsumi, T. and Motohashi, K. (1994). Application of rapid chloride permeability test to quality control of concrete, In: Durability of Concrete. ACI Special Publication-145, pp.487-502.

Mohammed, T., Yamaji, T. and Hamada, H. (2002). Microstructures and interfaces in concrete after 15 years of exposure in tidal environment, ACI Materials Journal, 99(4), pp.352-360.

Mojumdar, S., Chowdhury, B., Varshney, K. and Mazanec, K. (2004). Synthesis, moistureresistance, thermal, chemical and SEM analysis of macro-defect-free (MDF) cements. Journal of Thermal Analysis and Calorimetry, 78(1), pp.135-144.

Mora, J., Aguado, A. and Gettu, R. (2003). The influence of shrinkage reducing admixtures onplastic shrinkage. Materiales de Construccion, 53(271-272), pp.71-80.

Moranville-Regourd, M. (1998). Lea's Chemistry of Cement and Concrete. 4th ed. London:Arnold, pp.663-674.

Mori, H., Sudoh, G., Minegishi, K. and Ohta, T. (1974). Some properties of C-S-H gel formedby C3S hydration in the presence of alkali. In: 6th International Congress on the Chemistry of Cement. pp.2-12.

Naaman, A. and Reinhardt, H. (2003). Setting the stage: toward performance-based classification of FRC composites. In: Setting the stage: toward performance-based classification of FRC composites. RILEM S.A.R.L, pp.1-4.

Nanayakkara, A. (2003). Self-healing of Cracks in Concrete Subjected to Water Pressure. In:New Technologies for Urban Safety of Mega Cities in Asia. 
Neville, A. (1996). Properties of concrete. London: Pitman Pub.

Neville, A. (2002). Autogenous healing-a concrete miracle?. Concrete International, 24(11), 7682.

Nowak, A. and Szerszen, M. (2003). Life-Cycle Deterioration Models for Concrete Deck Slabs. In: Life-Cycle Performance of Deteriorating Structures. ASCE, pp.133-140.

Ostro, B. (1994). Estimating the health effects of air pollutants: A method with an application to Jakarta. Policy Research Working Paper. Washington, D.C: Policy Research Department.

Ozbay, E., Sahmaran, M., Yucel, H.E., Erdem, T.K., Lachemi, M. and Li, V.C. (2013). Effect of sustained flexural loading on self-healing of engineered Cementitious composites, Journal of Advanced Concrete Technology, 11(5), pp.167-179.

Powers, T. (1864). Topics in concrete technology 3: Mixtures containing intentionally entrained air. Journal of the PCA Development Laboratories, 6, pp.19-42.

Powers, T. (1865). Topics in concrete technology 3: Characteristics of air-void systems. Journal of the PCA Development Laboratories, 7, pp.23-41.

Qian, S. and Li, V. (2008). Simplified Inverse Method for Determining the Tensile Properties of Strain Hardening Cementitious Composites (SHCC). Journal of Advanced Concrete Technology, 6(2), pp.353-363.

Qian, S., Zhou, J., de Rooij, M., Schlangen, E., Ye, G. and van Breugel, K. (2009). Self-healing behavior of strain hardening cementitious composites incorporating local waste materials. Cement and Concrete Composites, 31(9), pp.613-621.

Ramm, W. and Biscoping, M. (1998). Autogenous healing and reinforcement corrosion of waterpenetrated separation cracks in reinforced concrete. Nuclear Engineering and Design, 179(2), pp.191-200.

Reinhardt, H. and Jooss, M. (2003). Permeability and self-healing of cracked concrete as a function of temperature and crack width. Cement and Concrete Research, 33(7), pp.981-985. 
Redon, C., Li, V., Wu, C., Hoshiro, H., Saito, T. and Ogawa, A. (2001). Measuring and Modifying Interface Properties of PVA Fibers in ECC Matrix. J. Mater. Civ. Eng., 13(6), pp.399-406.

Richardson, I. (2004). Tobermorite/jennite- and tobermorite/calcium hydroxide-based models forthe structure of C-S-H: applicability to hardened pastes of tricalcium silicate, $\beta$-dicalcium silicate, Portland cement, and blends of Portland cement with blast-furnace slag, metakaolin, or silica fume. Cement and Concrete Research, 34(9), pp.1733-1777.

Rokugo, K. (2005). Recent HPFRCC Research and Progress in Japan, International Workshop on High Performance Fiber Reinforced Cementitious Composites in Structural Applications.

Romualdi, J. and Mandel, J. (1964). Tensile strength of concrete affected by Unigormly Distributed and closely spaced short lengths of wire reinforcement, ACI Journal Proceedings, 61(6), pp.657671.

Roy, D. (1992). The effect of Blast Furnace Slag and Related Materials on the Hydration and Durability of Concrete. American Concrete Institute, pp.195-208.

Saadeghvaziri, M. and Hadidi, R. (2005). Transverse Cracking of Concrete Bridge Decks: Effects of Design Factors. J. Bridge Eng., 10(5), pp.511-519.

Sahmaran, M., Li, M. and Li, V. (2007). Transport properties of engineered Cementitious composites under chloride exposure, ACI Materials Journal, 104(6), pp.604-611.

Şahmaran, M. and Li, V. (2008). Durability of mechanically loaded engineered cementitious composites under highly alkaline environments. Cement and Concrete Composites, 30(2), pp.7281.

Şahmaran, M. and Li, V. (2008). Influence of microcracking on water absorption and sorptivity of ECC. Mater Struct, 42(5), pp.593-603.

Şahmaran, M. and Li, V. (2009). Durability properties of micro-cracked ECC containing high volumes fly ash. Cement and Concrete Research, 39(11), pp.1033-1043. 
Şahmaran, M. and Li, V. (2010). Engineered Cementitious Composites: Can They Be Accepted as a Crack-Free Concrete? Journal of Transportation Research Record, 39(11), pp.1033-1043.

Şahmaran, M., Yildirim, G. and Erdem, T. (2013). Self-healing capability of cementitiouscomposites incorporating different supplementary cementitious materials. Cement and Concrete Composites, 35(1), pp.89-101.

Şahmaran, M., Yildirim, G., Ozbay, E., Ahmed, K. and Lachemi, M. (2014). Self-healing ability of cementitious composites: effect of addition of pre-soaked expanded perlite. Magazine of Concrete Research, 66(8), pp.409-419.

Şahmaran, M., Yucel, H., Demirhan, S., Arik, M. and Li, V. (2012). Combined effect of aggregate and mineral Admixtures on tensile Ductility of engineered Cementitious composites, ACI Materials Journal, 109(6), pp.627-638.

Schiessl, P. and Brauer, N. (1996). Influence of Autogenous Healing of Cracks on Corrosion of Reinforcement. In: Durability of Building Materials and Components 7. pp.542-552.

Shah, S., Wan, K. and Weiss, W. (2000). Is High Strength Concrete Durable? In: Concrete Technology for a Sustainable Development in the 21st Century. pp.102-114.

Shi, C. (2004). Effect of mixing proportions of concrete on its electrical conductivity and the rapid chloride permeability test (ASTM C1202 or ASSHTO T277) results. Cement and Concrete Research, 34(3), pp.537-545.

Stark, J. and Ludwig, H. (1997). Influence of Water Quality on the Frost Resistance of Concrete. In: Freeze-Thaw Durability of Concrete. London: E \& FN Spon, pp.157-164.

Sukhotskaya, S., Mazhorova, V. and Terekhin, Y. (1983). Effect of autogenous healing of concrete subjected to periodic freeze-thaw cycles. Hydrotechnical Construction, 17(6), pp.294-296.

Taylor, H. (1997). Cement chemistry. London: T. Telford.

Ter Heide, N. and Schlangen, E. (2007). Self-healing of early age cracks in concrete. In: clProceedings of the First International Conference on Self Healing Materials. 
Ter Heide, N. (2005). Crack Healing in Hydrating Concrete. MSc. TU Delft.

Tsivilis, S., Tsantilas, J., Kakali, G., Chaniotakis, E. and Sakellariou, A. (2003). The permeability of Portland limestone cement concrete. Cement and Concrete Research, 33(9), pp.1465-1471.

Vaysburd, A., Brown, C., Bissonnette, B. and Emmons, P. (2004). "Realcrete” vs. "Labcrete”. Concrete International Journal, 26(2), pp.90-94.

Wang, S. and Li, V. (2007). Engineered Cementitious composites with high-volume fly ash, ACI Materials Journal, 104(3), pp.233-241.

Weiss, W. and Shah, S. (2002). Restrained shrinkage cracking: the role of shrinkage reducingadmixtures and specimen geometry. Mat. Struct. 35(2), pp.85-91.

Weimann, M. and Li, V. (2016). Drying Shrinkage and Crack Width of ECC. In: Brittle MatrixComposites-7. pp.37-46.

Wittmann, F. (1998). Separation of assignments: A new approach towards more durable reinforced concrete structures. In: Proceedings of the Fifth Workshop on Material Properties and Design, Durable Reinforced Concrete Structures. Aedificatio Publishers, pp.151-160.

Wua M., Johannesson B., Geiker M. 2012. A review of self-healing in cementitious materials and engineered cementitious composite as a self-healing material. Construction and Building Materials, 28: 571-583.

Yang, E., Sahmaran, M., Yingzi, Y. and Li, V. (2009). Rheological control in production of engineered Cementitious composites, ACI Materials Journal, 106(4), pp.357-366.

Yang, E., Yingzi, Y. and Li, V. (2007). Use of high volumes of fly ash to improve ECC mechanical properties and material greenness, ACI Materials Journal, 104(6), pp.620-628.

Yang, Y., Lepech, M. and Li, V. (2005). Self-healing of Engineered Cementitious Composites under Cyclic Wetting and Drying. In: Proceedings of Durability of Reinforced Concreteunder Combined Mechanical and Climatic Loads. pp.231-242. 
Yang, Y., Lepech, M., Yang, E. and Li, V. (2009). Autogenous healing of engineered cementitious composites under wet-dry cycles. Cement and Concrete Research, 39(5), pp.382-390.

Yang, Y., Yang, E. and Li, V. (2011). Autogenous healing of engineered cementitious composites at early age. Cement and Concrete Research, 41(2), pp.176-183.

Zhang, Z., Qian, S. and Ma, H. (2014). Corrigendum to "Investigating mechanical properties and self-healing behavior of micro-cracked ECC with different volume of fly ash" [Constr. Build. Mater. 52 (2014) 17-23]. Construction and Building Materials, 55, p.479.

Zhou, J., Qian, S., Sierra Beltran, M., Ye, G., van Breugel, K. and Li, V. (2009). Development of engineered cementitious composites with limestone powder and blast furnace slag. MaterStruct, 43(6), pp.803-814. 